\title{
Dilatant Strengthening as a Mechanism for Slow Slip Events
}

\section{Citation}

Segall, Paul, Allan M. Rubin, Andrew M. Bradley, and James R. Rice. 2010. Dilatant strengthening as a mechanism for slow slip events. Journal of Geophysical Research 115:B12305.

\section{Published Version}

doi:10.1029/2010JB007449

\section{Permanent link}

http://nrs.harvard.edu/urn-3:HUL.InstRepos:5026690

\section{Terms of Use}

This article was downloaded from Harvard University's DASH repository, and is made available under the terms and conditions applicable to Other Posted Material, as set forth at http:// nrs.harvard.edu/urn-3:HUL.InstRepos:dash.current.terms-of-use\#LAA

\section{Share Your Story}

The Harvard community has made this article openly available.

Please share how this access benefits you. Submit a story.

\section{Accessibility}




\title{
Dilatant strengthening as a mechanism for slow slip events
}

\author{
Paul Segall, ${ }^{1}$ Allan M. Rubin, ${ }^{2}$ Andrew M. Bradley, ${ }^{3}$ and James R. Rice ${ }^{4}$ \\ Received 10 February 2010; revised 14 June 2010; accepted 17 August 2010; published 3 December 2010.
}

[1] The mechanics of slow slip events (SSE) in subduction zones remain unresolved. We suggest that SSE nucleate in areas of unstable friction under drained conditions, but as slip accelerates dilatancy reduces pore pressure $p$ quenching instability. Competition between dilatant strengthening and thermal pressurization may control whether slip is slow or fast. We model SSE with 2-D elasticity, rate-state friction, and a dilatancy law where porosity $\phi$ evolves toward steady state $\phi_{s s}$ over distance $d_{c}$ and $\phi_{s s}=\phi_{0}+\epsilon \ln \left(v / v_{0}\right) ; v$ is slip speed. We consider two diffusion models. Membrane diffusion (MD) is approximated by $-\left(p-p^{\infty}\right) / t_{f}$ where $p$ and $p^{\infty}$ are shear zone and remote pore pressure and $t_{f}$ is a characteristic diffusion time. Homogeneous diffusion (HD) accurately models fault-normal flow with diffusivity $c_{\text {hyd }}$. For MD, linearized analysis defines a boundary $\mathcal{E}=1-a / b$ between slow and fast slip, where $\mathcal{E} \equiv f_{0} \epsilon / \beta b\left(\sigma-p^{\infty}\right), f_{0}, a$, and $b$ are friction parameters and $\beta$ is compressibility. When $\mathcal{E}<1-a / b$ slip accelerates to instability for sufficiently large faults, whereas for $\mathcal{E}>1-a / b$ slip speeds remain quasi-static. For $\mathrm{HD}, E_{p} \equiv$ $\epsilon h /\left(\beta\left(\sigma-p^{\infty}\right) \sqrt{v^{\infty} / c_{h y d} d_{c}}\right)$ defines dilatancy efficiency, where $h$ is shear zone thickness and $v^{\infty}$ is plate velocity. SSE are favored by large $\epsilon h$ and low effective stress. The ratio $E_{p}$ to thermal pressurization efficiency scales with $1 /\left(\sigma-p^{\infty}\right)$, so high $p^{\infty}$ favors SSE, consistent with seismic observations. For $E_{p} \sim 10^{-3}$ transient slip rates, repeat times, average slip, and stress drops are comparable to field observations. Model updip propagation speeds are comparable to those observed along-strike. Many simulations exhibit slow phases driven by steady downdip slip and faster phases that relax the accumulated stress. Model SSE accommodate only a fraction of plate motion; the remaining deficit must be accommodated during coseismic or postseismic slip.

Citation: Segall, P., A. M. Rubin, A. M. Bradley, and J. R. Rice (2010), Dilatant strengthening as a mechanism for slow slip events, J. Geophys. Res., 115, B12305, doi:10.1029/2010JB007449.

\section{Introduction}

[2] One of the most exciting discoveries in solid earth geophysics in recent decades has been the recognition that many subduction zones undergo transient slip events at depths below the locked megathrust zone. These slip events, which were first detected by GPS networks, have been found in Cascadia [Dragert et al., 2001; Miller et al., 2002; Szeliga et al., 2008], southwest Japan [e.g., Hirose et al., 1999; Miyazaki et al., 2006], Mexico [Kostoglodov et al., 2003; Larson et al., 2007], New Zealand [Douglas et al., 2005; McCaffrey et al., 2008; Delahaye et al., 2009], and Alaska [Ohta et al., 2006]. Slow slip has also been found along the San Andreas Fault [Linde

\footnotetext{
${ }^{1}$ Geophysics Department, Stanford University, Stanford, California, USA.

${ }^{2}$ Department of Geosciences, Princeton University, Princeton, New Jersey, USA.

${ }^{3}$ Computational and Mathematical Engineering, Stanford University, Stanford, California, USA.

${ }^{4}$ Department of Earth and Planetary Sciences and School of Engineering and Applied Sciences, Harvard University, Cambridge, Massachusetts, USA.

Copyright 2010 by the American Geophysical Union. 0148-0227/10/2010JB007449
}

et al., 1996; Murray and Segall, 2005], and on the decollement beneath Kilauea volcano [Cervelli et al., 2002; Segall et al., 2006; Brooks et al., 2006; Montgomery-Brown et al., 2009]. In Cascadia slow slip events are periodic with interevent times, varying with latitude, of from 11 to 18 months [Brudzinski and Allen, 2007]. In southwest Japan, periods of roughly 6 months, 1 year, and 6 years have been observed.

[3] Transient slip in subduction zones is often, but apparently not always, accompanied by tectonic tremor [Obara, 2002; Rogers and Dragert, 2003; Obara et al., 2004]. The periodic recurrence and accompanying seismic signature has led to the designation Episodic Tremor and Slip (ETS). Shelly et al. [2007] showed that tremor in southwest Japan contains locatable events, termed low-frequency earthquakes (LFE), and that the tremor consists largely, and possibly completely, of repeated excitation of LFE sources. The lowfrequency events there locate on the subducting plate interface [Shelly et al., 2006]. Ide et al. [2007] showed that the LFEs have focal mechanisms consistent with slip on the plate interface in the plate convergence direction. Together these observations argue strongly that tremor, at least in the Nankai region, is caused by slip on the plate interface.

[4] The mechanism of nonvolcanic tremor in Cascadia has been more controversial, with early locations placing 
significant tremor well above the subducting plate interface [Kao et al., 2005]. However, recent work has shown that the Cascadia tremor contains discrete events located on the megathrust [Brown et al., 2009]. In addition, S minus $\mathrm{P}$ times within tremor directly beneath seismic arrays in Cascadia also place the tremor source near the plate interface [ La Rocca et al., 2009]. The weight of evidence thus may be shifting to the view that tremor in Cascadia is also caused by slip on the plate interface. It should also be noted that tremor is observed between the major ETS events, and tends to be located somewhat deeper than the ETS tremor [Wech et al., 2009].

[5] There are, however, cases where slow slip events have been detected geodetically without observable tremor, including New Zealand [Delahaye et al., 2009], and the Boso Peninsula of Japan [Sagiya, 2004; Ozawa et al., 2003, 2007]. It thus appears that tremor is not necessary for slow slip to occur. However, the clear spatial and temporal association of these signals in Cascadia and Nankai demonstrate that they are intimately linked in these regions. Nonvolcanic tremor has not yet been found during slow slip beneath Kilauea volcano; however these events are associated with swarms of small, high-frequency earthquakes [Segall et al., 2006; Brooks et al., 2006; Montgomery-Brown et al., 2009]. Microseismicity has also been observed with slow slip events beneath the Boso Peninsula [Sagiya, 2004; Ozawa et al., 2003, 2007], Imperial Valley [Lohman and McGuire, 2007], and the north island of New Zealand [Delahaye et al., 2009].

[6] Understanding the physics of slow slip events and how they differ from normal, high-frequency earthquakes is one of the most pressing current challenges in seismology. Critical observations that must be explained by any viable model of slow slip include the following:

[7] 1. Minimum dimensions of slow slip zones are typically several tens of kilometers.

[8] 2. Maximum slip in a single event is typically small ( $\sim 2 \mathrm{~cm}$ in Cascadia).

[9] 3. These observations imply low static stress drops. For a width of $60 \mathrm{~km}$, slip of $2 \mathrm{~cm}$, and $\mu /(1-\nu)$ of $4 \times 10^{4} \mathrm{MPa}$ ( $\mu$ is the shear modulus and $\nu$ Poisson's ratio), the stress drop is on the order of $0.01 \mathrm{MPa}$.

[10] 4. Average slip velocities are roughly $1-2$ orders of magnitude above the plate velocity $\left(2 \mathrm{~cm} / 10\right.$ days $\simeq 10^{-8} \mathrm{~m} / \mathrm{s}$ in Cascadia, versus $10 \mathrm{~cm} / 2$ days $\simeq 5 \times 10^{-7} \mathrm{~m} / \mathrm{s}$ beneath Kilauea).

[11] 5. Slow slip event durations vary from roughly 2 days at Kilauea [Cervelli et al., 2002; Montgomery-Brown et al., 2009] to on the order of 6 years for the Tokai slow slip events in Japan [Miyazaki et al., 2006].

[12] 6. The repeat period between events is commonly near 1 year, although both shorter and longer intervals are observed.

[13] 7. Slow slip events in Cascadia propagate along strike at rupture speeds of $\sim 10 \mathrm{~km} / \mathrm{d}$ [e.g., Schwartz and Rokosky, 2007], while others such as the Tokai slow event slip for several years in largely the same locality [Miyazaki et al., 2006].

[14] 8. High ratios of compressional to shear wave velocity imaged in tomographic and/or receiver function studies [Kodaira et al., 2004; Shelly et al., 2006; Audet et al., 2009] have been interpreted as indicative of high ambient pore pressures in regions where slow slip events occur.
[15] Several classes of models to explain slow slip have been explored. Some workers have posited a change in frictional behavior from velocity weakening at low slip speeds to velocity strengthening at higher rates [e.g., Shibazaki and Iio, 2003; Shibazaki and Shimamoto, 2007]. Such behavior has been reported for simulated halite fault gouges [Shimamoto, 1986], and for chrysotile and lizardite serpentine at temperatures below $200^{\circ} \mathrm{C}$ [Moore et al., 1997]. However, these phases are not stable at temperatures above $250-300^{\circ} \mathrm{C}$ [Moore et al., 1997]. Antigorite, the serpentine mineral stable at from $200^{\circ} \mathrm{C}$ to $500-600^{\circ} \mathrm{C}$, exhibits velocity strengthening behavior over the range of temperatures and velocity steps tested, as do the layer silicates brucite and talc [Moore and Lockner, 2007]. Thus, the notably limited data available for mafic rocks under appropriate pressure and temperature conditions does not presently support this hypothesis, although further laboratory testing is certainly warranted.

[16] Another class of model [Liu and Rice, 2005a, 2007] exploits the fact that faults with rate and state friction exhibit oscillatory behavior near neutral stability. This is illustrated most simply by spring slider systems. Ruina [1983] showed that such systems are linearly unstable with respect to perturbations from steady sliding if the spring stiffness is less than a critical value, $k_{c r i t}=\left(\sigma-p^{\infty}\right)(b-a) / d_{c}$. Here $\left(\sigma-p^{\infty}\right)$ is the effective normal stress, $a$ and $b$ are rate and state constitutive parameters, defined in equation (2) below, and $d_{c}$ is the characteristic slip distance for state evolution (see equation (3)). For spring stiffness equal to $k_{c r i t}$ small perturbations result in sustained oscillations, whereas for $k>$ $k_{c r i t}\left(k<k_{c r i t}\right)$ perturbations from steady sliding decay (grow). In an elastic continuum, the effective stiffness of a slip patch decreases with the size of the patch. For plane strain deformation the stiffness at the patch center is $C[\mu /(1-\nu)] / L$, where $\mu$ is the shear modulus, $\nu$ is Poisson's ratio, $L$ is the patch length, and the coefficient $C$, of order unity, depends upon the distribution of stress drop or slip. For a uniform stress drop $C=1$; for a periodic stress drop on an infinitely long fault $C=2 / \pi$. Equating this stiffness to $k_{c r i t}$ leads to a critical dimension for nucleation given by

$$
h^{*}=C \frac{d_{c} \mu /(1-\nu)}{\left(\sigma-p^{\infty}\right)(b-a)} .
$$

For $C=2 / \pi$ this is consistent with $h^{*}$ defined by Rice [1993]; we use $C=1$ when reporting normalized fault dimensions later in this paper.

[17] Translating the critical stiffness concept into a nucleation length for faults in elastic continua is nontrivial because in general the fault stiffness varies in both space and time. One manifestation of this is that, unlike spring slider systems with $k$ slightly below $k_{c r i t}$, faults modestly longer than $h^{*}$ do not accelerate to instability. Instead, there is a range of fault lengths larger than $h^{*}$ that exhibit stable, oscillatory departures from steady sliding. One way of rationalizing this is to note that for a fixed length fault with uniform stress drop, the stiffness (stress drop per slip) is greater near the ends than at the center. For a fault modestly longer than $h^{*}$ with pinned ends this leads to a gradual shrinking of the accelerating region, a negative feedback that further increases the stiffness and ultimately leads to decelerating slip. With unpinned ends, however, the "aging" form of the state evolution law (defined below), and laboratory values of $a / b$, the 

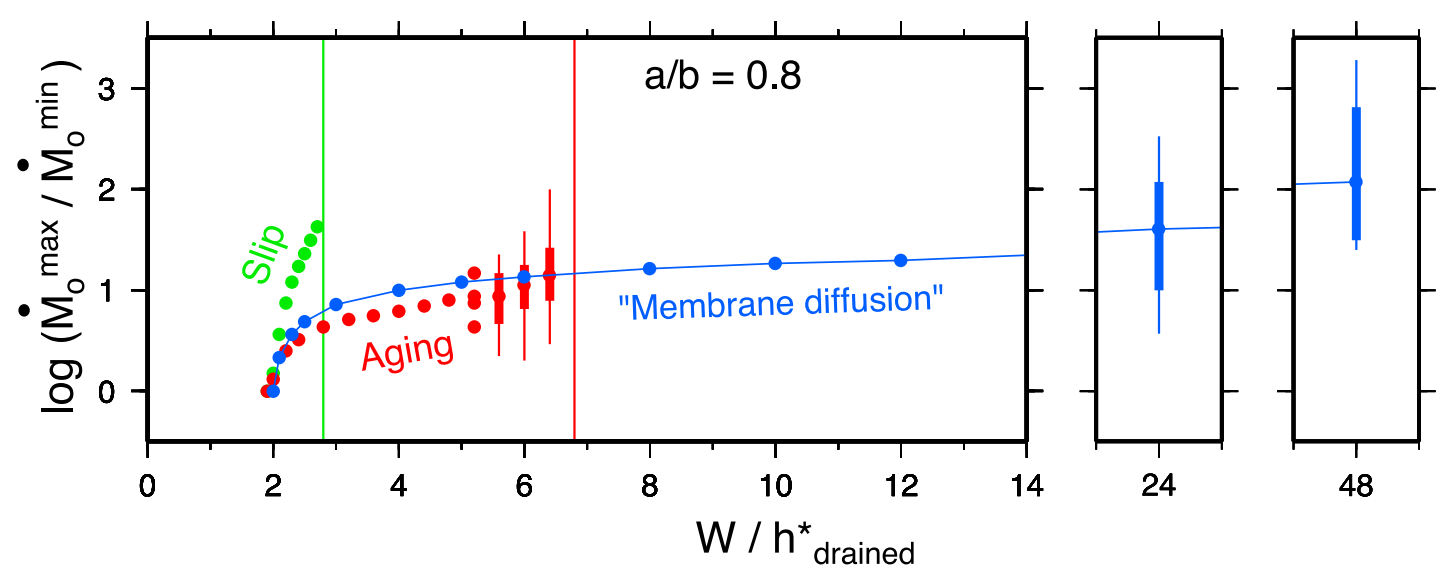

Figure 1. Ratio of maximum to minimum moment rate as a function of length of the velocity weakening zone $W$, normalized by drained critical nucleation dimension $h_{\text {drain }}^{*}$. These computations include a velocity strengthening region (with $a / b=1.2$ ) of length $4 W$ between the velocity weakening zone and the fixed slip rate boundary condition. Drained behavior with slip law (green), aging law (red), and slip law with membrane diffusion (blue). Single dots show periodic behavior, while fat and thin lines show the middle $50 \%$ and $80 \%$, respectively, when the behavior is aperiodic. Vertical green and red lines show the onset of dynamic instability for the slip and aging laws, respectively. $d_{c}=40 \mu \mathrm{m}, b=0.01$, and $v^{\infty}=10^{-9} \mathrm{~m} / \mathrm{s}$. For membrane diffusion calculations $\mathcal{E}=0.6$ and $v^{\infty} t_{f} / d_{c}=1$.

large stress concentrations at the margins of the slipping zone cause the nucleation zone to expand such that it asymptotically approaches a length larger than $h^{*}($ with $C=1)$ by a factor of $(2 / \pi)(1-a / b)^{-1}[$ Rubin and Ampuero, 2005] (note that this is twice the half-length $L_{\infty}$ defined there).

[18] For the aging law form of state evolution, stable oscillatory slip can thus occur over a range of fault lengths from roughly $h^{*}$ to $2 L_{\infty}$. This range becomes quite large as $a / b$ approaches 1 ; that is, for faults that are near velocityneutral. However, for the "slip" law form of state evolution numerical simulations show that nucleation occurs as a singlesided slip pulse that reaches dynamic slip speeds at a length that is several times smaller than $2 L_{\infty}$ [Ampuero and Rubin, 2008; Rubin and Ampuero, 2009]. Thus, the range of fault lengths that exhibit stable oscillatory slip is significantly smaller with the slip law than it is for the aging law.

[19] In short, for slow slip events to arise from rate-state friction effects near neutral stability with either evolution law the dimension of the fault participating in the slow slip event must be only modestly larger than the critical dimension for nonsteady slip to nucleate. If the slip zone is smaller than $h^{*}$, slip is steady and no transients occur; if the slip zone is too much larger than $h^{*}$, slip becomes dynamically unstable. For laboratory values of friction parameters and normal stresses of order $100 \mathrm{MPa}, h^{*}$ is of the order of meters, far too small to reconcile with the order $10 \mathrm{~km}$ minimum dimension of slow slip events. Kuroki et al. [2004] find models of transient slip with appropriate spatial dimensions by choosing slip weakening distances, $d_{c}, 3-4$ orders of magnitude larger than observed in laboratory experiments.

[20] Alternatively, Liu and Rice [2007] suggest that low effective normal stresses within slow slip zones (i.e., highly elevated pore pressure $p$ there, which they inferred from petrologic constraints on seafloor dehydration and seismic studies of compressional to shear speed ratios) cause $h^{*}$ to be sufficiently large to explain geodetic observations. These authors model simulated transient slip events that have durations and interevent times comparable to what has been observed in the Cascade subduction zone. Episodic slip occurs in the transition between the locked megathrust zone and the velocity strengthening fault at greater depth. Liu and Rice [2009] extend this work, considering laboratory data for gabbro that indicate a transition from velocity weakening to velocity strengthening friction at a considerably higher temperature than for granite, which was used in previous studies. Mapping these data to depth using thermal models for the Cascade subduction zone places the transient slip events at a depth range more compatible with geodetic observations than do the granite results. In these models, the width of the transition zone, referred to as $W$, must be larger than $h^{*}$, but not so large that the slip becomes dynamically unstable. For the aging law form of the state evolution equations employed by Liu and Rice [2007] the ratio $W / h^{*}$ can be up to $\sim 7$ for spatially uniform $a / b=0.8$ before the slip becomes dynamically unstable (Figure 1). For the nonuniform distribution of $a / b$ adopted by Liu and Rice [2007] stable oscillatory slip occurs for a reasonably broad range of fault widths, roughly $1.4 \leq W / h^{*} \leq 10$, suggesting that such behavior has a reasonable chance of occurring in nature. This broad range derives from the large increase in apparent fracture energy with increasing slip speed implied by the aging law, an increase that derives from an increase in the effective slipweakening distance with increasing slip speed [Rubin and Ampuero, 2005]. However, no laboratory data support this behavior [Nakatani, 2001].

[21] Rather, laboratory experiments indicate that in response to a step increase in sliding velocity the effective slip-weakening distance is independent of the magnitude of the velocity increase, an observation consistent with the slip law [Ruina, 1980; Bayart et al., 2006] (although the slip law is less successful, compared to the aging law, in representing restrengthening in nominally stationary contact [Beeler et al., 1994; Marone, 1998]). Calculations similar to those of Liu and Rice [2007] employing the slip law show that the 
maximum value of $W / h^{*}$ allowing stable slip is only 3 for $a / b=0.8$ (Figure 1) and 5 for $a / b=0.9$ [Rubin, 2008]. Because the two evolution laws are asymptotically identical near steady state, the minimum values of $W / h^{*}$ allowing oscillatory slip ( $\sim 2$ for the boundary conditions of Figure 1 and $a / b=0.8 ; \sim 2.5$ for $a / b=0.9$ ) are the same for both laws. Thus, the range of $W / h^{*}$ allowing oscillatory slip is only 1.5 and 2 for the slip law and the aforementioned values of $a / b$. For this reason it is difficult for models employing rate and state friction alone to explain the observations, especially with the best laboratory-supported state evolution law for describing response to abrupt increases in slip rate. The dimensions of slow slip zones would have to be within an extremely narrow range in order to generate a significant transient without becoming dynamically unstable. It appears that an additional strengthening mechanism is required to explain the common occurrence of stable slow slip in nature. Based on prior studies of dilatancy in slip stabilization [Segall and Rice, 1995; Taylor, 1998, chapter 6; Taylor and Rice, 1998; Segall and Rubin, 2007], Liu and Rice [2007, 2009] suggested that its consideration might expand the stable range of $W / h^{*}$, and hence the spatial extent $W$ of their predicted stable slip zone, in a manner as required to better fit observations from Cascadia. However, they did not model and quantify the dilatancy effect except for a preliminary study [Liu and Rice, 2005b] showing that what we call the "membrane diffusion model" here did reduce along-strike propagation speeds of episodic slip events in 3-D subduction simulations.

[22] An alternative model was offered by Perfettini and Ampuero [2008], who explored the possibility that slow slip events occur in regions of steady state velocity strengthening friction $(a>b)$, with transient slip induced by external stress perturbations. They further suggest that pore pressure transients due to so-called "fault valve" behavior could provide the requisite external forcing. In this model the periodicity of slow slip is controlled by the period of the fault valve phenomenon.

[23] Here we explore the possibility that dilatancy provides the additional stabilization required to expand the permissible range for slow slip events to occur in velocity weakening regions. The hypothesis is that frictional weakening allows slip to nucleate under drained conditions, but that as the slip rate increases the fault becomes increasingly undrained. Depending on constitutive parameters and the ambient effective normal stress, dilatancy can quench the instability resulting in a slow slip event. Dilatant stabilization is not a new concept, having been extensively studied in the context of slip-weakening friction by Rice [1975], Rice and Simons [1976], and Rudnicki [1979], among others. Dilatant stabilization has also been suggested as a mechanism for stabilizing some landslides [Schulz et al., 2008]. Segall and Rice [1995] combined dilatancy and mechanical compaction with rate-state friction in single-degree-offreedom spring slider systems. Taylor [1998, chapter 6] extended this work to two-dimensional continuum models of subduction zones with an approximate diffusion model. He showed that dilatancy can limit the updip extent of dynamic ruptures such that they do not reach the trench, as predicted in the absence of dilatancy. Hillers and Miller [2006] extend this work to two dimensional faults, retaining the simplified diffusion model. They find instabilities for drained behavior, stable sliding for undrained behavior, and transient aseismic slip for intermediate behavior. Segall and Rubin [2007] showed that propagating slow slip events can occur for appropriate parameter range with the approximate diffusion model, while Segall et al. [2008] showed that such behavior extends to the more accurate homogeneous diffusion case, employing finite difference calculations. The latter further suggest that whether slip is slow or fast depends on whether or not dilatancy limits slip to speeds below those at which thermal weakening effects dominate; Segall and Rice [2006], Schmitt et al. [2007], and Schmitt and Segall [2008] show that above a critical slip speed thermal pressurization dominates rate-state friction during earthquake nucleation. Suzuki and Yamashita [2009] consider the same hypothesis in the context of slip-weakening friction and with a different dilatancy formulation. They show slow ruptures occur when the ambient effective normal stress is sufficiently low consistent with results presented here; however, their computations are limited to integration times of order of seconds, such that results are dependent on assumed initial conditions.

\section{Governing Equations}

[24] Laboratory experiments show that the frictional resistance depends on the instantaneous slip speed $v$ and the past sliding history, which can be characterized by an internal state variable $\theta$,

$$
\tau=(\sigma-p)\left[f_{0}+a \ln \frac{v}{v_{0}}+b \ln \frac{\theta v_{0}}{d_{c}}\right]
$$

[Ruina, 1983; Kilgore et al., 1993]. Here $a$ and $b$ are material constants, $v_{0}$ is a normalizing constant, and $f_{0}$ is the nominal friction. The state is sometimes interpreted as the average asperity contact lifetime, and evolves over a characteristic displacement $d_{c}$. The proper mathematical description of state evolution has not been fully resolved (and may not be fully described by any simple analytical representation), although two forms in wide use are

$$
\begin{aligned}
& \frac{d \theta}{d t}=1-\frac{\theta v}{d_{c}} \\
& \frac{d \theta}{d t}=-\frac{\theta v}{d_{c}} \ln \left(\frac{\theta v}{d_{c}}\right) .
\end{aligned}
$$

The first exhibits healing in stationary contact and is thus referred to as the "aging" law. In the second form state evolves only with slip ( $d \theta / d t$ vanishes when $v=0)$, and is thus referred to as the "slip law." In both cases the steady state value of $\theta$ is $d_{c} / v$. Laboratory studies inevitably indicate strengthening with increased time of stationary contact [Dieterich and Kilgore, 1994; Beeler et al., 1994], indicating that the aging law is more consistent with data when $\theta$ is far below steady state as it must be between SSE episodes. However, velocity stepping tests exhibit a symmetric stress versus slip response to step increases and decreases in loading velocity. In addition, the distance scale over which stress decays to steady state following a step velocity increase, when $\theta$ is far above steady state, is nearly independent of the magnitude of the velocity step. Both features are consistent with the slip law but not the aging law [Ruina, 1983; Bayart et al., 2006]. Because nucleation is most sensitive to fault 


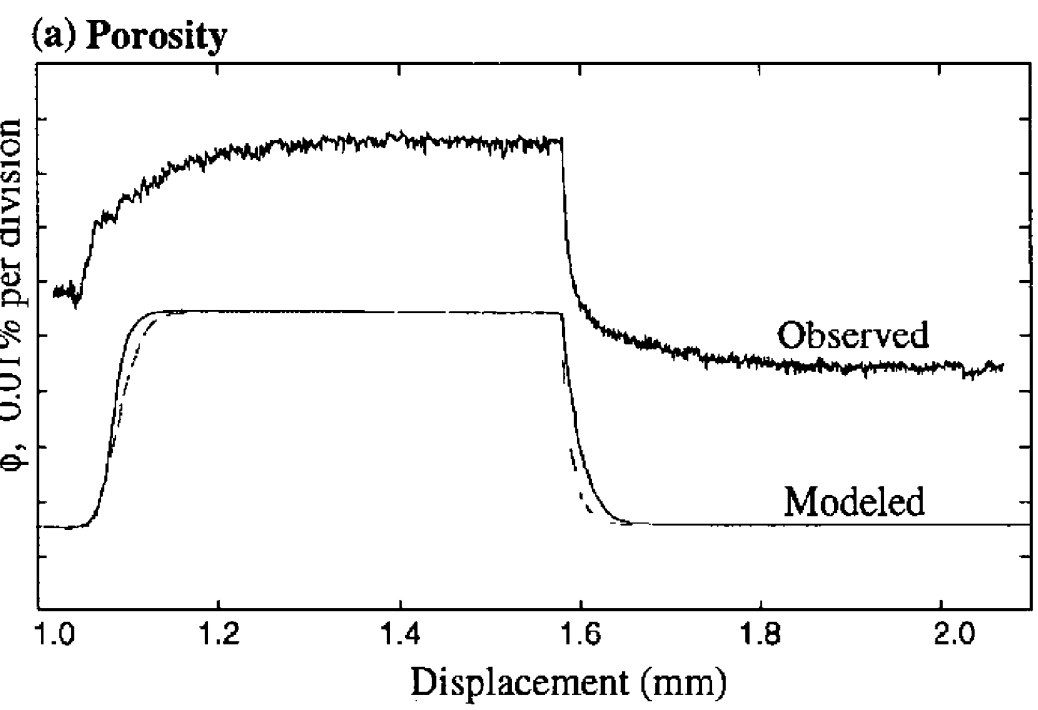

(b) Coefficient of Friction

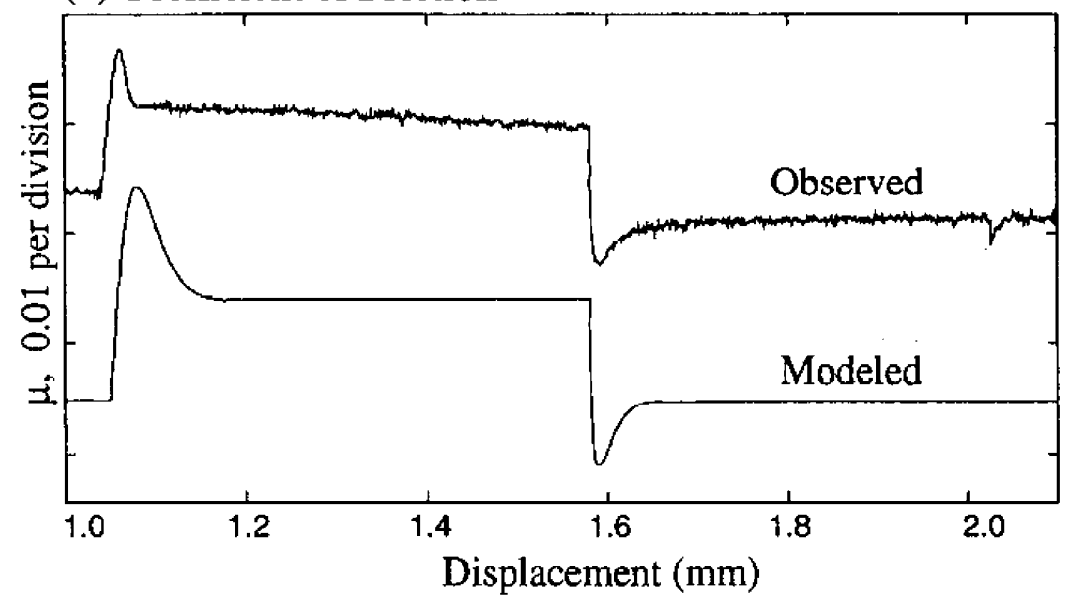

Figure 2. Change in porosity and friction upon step changes in slip rate. Data from Marone et al. [1990] at $150 \mathrm{MPa}$ confining stress. (a) Change in porosity after removing long-duration slip-dependent variations. The slip speed first increases from 1 to $10 \mu \mathrm{m} / \mathrm{s}$ and then drops back to $1 \mu \mathrm{m} / \mathrm{s}$. Fit to the porosity evolution using a constitutive law of the form of equation (4) (dashed line), with $\epsilon=1.7 \times 10^{-4}$ and $d_{c}=20$ microns. An alternate formulation is shown as the solid line. (b) Change in friction fit with the same value of $d_{c}$ and $a=0.01$ and $b=0.006$. From Segall and Rice [1995].

behavior near to and well above steady state [Ampuero and Rubin, 2008], the slip law appears to be the more relevant one for nucleation, although it may bias the state at which nucleation begins after a prior event.

[25] Following Segall and Rice [1995] we assume a constitutive equation for the inelastic change in porosity $\delta \phi$, including both dilatancy and compaction, motivated in part by experiments of Marone et al. [1990]. In particular, we associate dilatancy/compaction with changes in the average lifetime of asperity contacts within the fault gouge, such that

$$
\begin{gathered}
\delta \phi=-\epsilon \ln \left(\frac{v_{0} \theta}{d_{c}}\right) \\
\frac{d \phi}{d t}=-\epsilon \frac{d}{d t} \ln \left(\frac{v_{0} \theta}{d_{c}}\right)=-\frac{\epsilon}{\theta} \frac{d \theta}{d t},
\end{gathered}
$$

where $\epsilon$ is an empirically derived constant of order $10^{-4}$, based on Marone et al.'s [1990] experiments. Above steady state, that is for $\theta>d_{c} / v, \theta$ decreases (from (3)), and the gouge dilates, while below steady state, $\theta$ increases and the gouge compacts (Figure 2). Sleep [1997] considered a modification of this constitutive law in which compaction saturates when $\theta$ is far below steady state.

[26] The fault is taken to lie in the plane $y=0$. We employ the radiation damping approximation of elastodynamics [Rice, 1993] such that stress equilibrium on the fault becomes

$$
\frac{\mu}{2 \pi(1-\nu)} \int_{-\infty}^{\infty} \frac{\partial \delta / \partial \xi}{\xi-x} d \xi-f(v, \theta)(\sigma-p)=\frac{\mu}{2 v_{s}} v
$$

where the difference between the elastic stress and the frictional resistance is balanced by the stress change associated with plane shear waves (with velocity $v_{s}$ ) radiating from the 
fault. The first term on left represents the elastic stress due to gradients in slip $\delta$, while the second term is the frictional resistance. Note that for antiplane geometry we simply replace $\mu /(1-\nu)$ with $\mu$. Lapusta et al [2000] show that this "quasi-dynamic" formulation seems to lead to a reasonable representation of dynamic slip, although maximum slip speeds and propagation rates are underpredicted relative to the full elastodynamic results.

[27] To investigate the role of dilatancy in slow slip events we consider coupled friction, dilatancy and pore fluid flow. Neglecting conduction parallel to the fault (the $x$ direction) and heat advection in the pore fluid phase [see Lachenbruch, 1980], and assuming spatially uniform thermal properties, the temperature field follows

$$
\frac{\partial \mathcal{T}}{\partial t}=c_{t h} \frac{\partial^{2} \mathcal{T}}{\partial y^{2}}+\frac{\tau \dot{\gamma}}{\rho c}
$$

[e.g., Rice, 2006]. Here, $\dot{\gamma}$ is the shear strain rate, $c$ is specific heat capacity and $c_{t h}$ is thermal diffusivity. For a thermal diffusivity of $10^{-6} \mathrm{~m}^{2} / \mathrm{s}$, a thermal anomaly penetrates on the order of a few meters in the 1 year cycle time for typical slow slip events. Compared to the tens of kilometers characteristic dimensions of the slow slip events, this indicates that gradients in the along-fault direction are likely to be extremely small compared to the across-fault direction. The actively shearing zone, for which $\dot{\gamma}$ is nonzero, is assumed to have uniform thickness $h$. For times greater than the characteristic diffusion time across the layer (at most a few seconds) the limit $h \rightarrow 0$ is sensible, in which case (7) reduces to [Rice, 2006],

$$
\frac{\partial \mathcal{T}}{\partial t}=c_{t h} \frac{\partial^{2} \mathcal{T}}{\partial y^{2}} ;\left.\quad \frac{\partial \mathcal{T}}{\partial y}\right|_{y=0^{ \pm}}=\mp \frac{\tau v}{2 c \rho c_{t h}} .
$$

[28] Neglecting pore fluid flow parallel to the fault, for the same reason that heat flow in this direction is negligible, changes in pore pressure in the rock surrounding the shear zone is given by

$$
\frac{\partial p}{\partial t}=\frac{1}{\eta \beta} \frac{\partial}{\partial y}\left(\kappa \frac{\partial p}{\partial y}\right)+\Lambda \frac{\partial \mathcal{T}}{\partial t} \quad y<-h, y>0
$$

where $\eta$ is pore fluid viscosity, $\beta$ is compressibility of the fluid and the pore space, and $\kappa$ is the permeability. $\Lambda$ is the thermal pressurization parameter, equal to the ratio of thermal expansivity to compressibility [e.g., Segall and Rice, 2006, equation 19]. For spatially uniform permeability, the transport term can be written in terms of the hydraulic diffusivity $c_{\text {hyd }}=\kappa / \eta \beta$. Within the shear zone, conservation of fluid mass, Darcy's law, and a constitutive equation for the fault gouge [e.g., Segall and Rice, 2006, equation 21] (correcting a sign error there) yield

$$
\frac{\partial p}{\partial t}+\frac{\dot{\phi}}{\beta}-\Lambda \frac{\partial \mathcal{T}}{\partial t}=\left.\frac{2 c_{h y d}}{h} \frac{\partial p}{\partial y}\right|_{y=0^{+}} \quad-h<y<0,
$$

where $c_{\text {hyd }}$ is the hydraulic diffusivity of the rock adjacent to the shearing zone, and $p, \mathcal{T}$ and $\dot{\phi}$ on the left are averages across that layer. (For times that are long compared to the characteristic diffusion times across the layer, variations of $p$ and $\mathcal{T}$ with $y$ within it are negligible.) Equation (10) shows that, as expected, dilatancy acts as a fluid pressure sink, whereas an increase in $\mathcal{T}$ acts as a pressure source. Assuming, due to the constraint of the bordering material, that dilatancy (and compaction) act only normal to the plane of the fault it can be shown that $h \dot{\phi}=(1-\phi) \dot{h}$, where $\dot{h}$ is the change in thickness of the shearing layer. Indeed in many experimental studies of fault gouge dilation it is $\dot{h}$ that is actually measured. Multiplying both sides of (10) by $h$, the left hand side becomes $h \dot{p}+(1-\phi) \dot{h} / \beta-h \Lambda \dot{\mathcal{T}}$. We assume dilatancy greatly dominates effects of $p$ and $\mathcal{T}$ variation on porosity change within the thin shearing layer, so that the $\dot{p}$ and $\Lambda \dot{\mathcal{T}}$ terms can be neglected compared to that with $\dot{h}$. It is convenient to then formally take the limit $h \rightarrow 0$, with $h \dot{\phi}$ remaining finite, so that the left-hand side reduces to $(1-\phi) \dot{h} / \beta$. Thus, in this limit, and assuming uniform hydraulic properties in $y>0$, equations (9) and (10) reduce to

$$
\frac{\partial p}{\partial t}=c_{h y d} \frac{\partial^{2} p}{\partial y^{2}}+\Lambda \frac{\partial \mathcal{T}}{\partial t} ;\left.\quad \frac{\partial p}{\partial y}\right|_{y=0^{+}}=\frac{(1-\phi) \dot{h}}{2 \beta c_{h y d}}=\frac{h \dot{\phi}}{2 \beta c_{h y d}} .
$$

\subsection{Dimensional Analysis}

[29] To clarify the role of thermal pressurization relative to dilatant strengthening, we explore a nondimensionalization of equations (8) and (11). Specifically, define nondimensional variables, $\tilde{v}=v / v^{\infty}, \tilde{t}=t v^{\infty} / d_{c}, \tilde{\theta}=\theta v^{\infty} / d_{c}, \tilde{p}=p /\left(\sigma-p^{\infty}\right), \tilde{\tau}=$ $\tau / f_{0}\left(\sigma-p^{\infty}\right)$, and $\tilde{T}=\Lambda \mathcal{T} /\left(\sigma-p^{\infty}\right)$. The fault perpendicular distance is normalized by the characteristic fluid diffusion distance such that, $\tilde{y}=\sqrt{y^{2} v^{\infty} / c_{h y d} d_{c}}$. This leads to a heat equation in which the thermal diffusivity is scaled by $c_{\text {hyd }}$, with boundary condition

$$
\begin{aligned}
\left.\frac{\partial \tilde{\mathcal{T}}}{\partial \tilde{y}}\right|_{y=0} & =-E_{T} \tilde{\tau} \tilde{v} \\
E_{T} & =\frac{f_{0} \Lambda}{2 \rho c} \sqrt{\frac{c_{h y d} d_{c} v^{\infty}}{c_{t h}^{2}}} .
\end{aligned}
$$

The nondimensional pore pressure equation (11) now has unit coefficients, with boundary condition

$$
\begin{aligned}
\left.\frac{\partial \tilde{p}}{\partial \tilde{y}}\right|_{y=0} & =-E_{p} \frac{\dot{\tilde{\theta}}}{\tilde{\tilde{\theta}}} \\
E_{p} & =\frac{\epsilon}{2 \beta\left(\sigma-p^{\infty}\right)} \sqrt{\frac{h^{2} v^{\infty}}{c_{h y d} d_{c}}} .
\end{aligned}
$$

Dilatancy acts to decrease pore pressure thereby stabilizing slip, while thermal pressurization increases pore pressure and thus acts to destabilize slip. Of particular interest for understanding the tendency for slow versus fast slip, is the ratio of dilatancy to shear heating efficiency,

$$
\frac{E_{p}}{E_{T}}=\frac{\rho c}{f_{0} \Lambda \beta\left(\sigma-p^{\infty}\right)}\left(\frac{\epsilon h}{d_{c}}\right)\left(\frac{c_{t h}}{c_{h y d}}\right) .
$$

Equation (14) shows that slow slip is favored by strong dilatancy (large $\epsilon h / d_{c}$ ), low compressibility, and low effective stress. The latter result is easily understood; high 
effective normal stress leads to high rates of heat production. In contrast, the rate of pore pressure change due to dilatancy is independent of effective normal stress, but scales inversely with the compressibility, equation (10).

\subsection{Thermal Weakening During Slow Slip}

[30] Segall and Rice [2006], Schmitt et al. [2007], and Schmitt and Segall [2008] examined the relative importance of thermal pressurization and rate-state friction during earthquake nucleation, ignoring dilatancy effects. These studies demonstrate that for permeabilities associated with active crustal faults thermal weakening dominates rate- and statedependent weakening at slip speeds in excess of roughly $10^{-4} \mathrm{~m} / \mathrm{s}$, to $10^{-2} \mathrm{~m} / \mathrm{s}$, depending on material parameters, particularly hydraulic diffusivity. These results suggest that thermal pressurization is unlikely to be dominant during slow slip events with characteristic slip rates of $10^{-7} \mathrm{~m} / \mathrm{s}$ or less. Thus, for simplicity we assume in the remainder of the discussion that the fault remains isothermal, an approximation that will be violated if slip rates become too high. Work in progress combines both dilatancy and thermal pressurization effects [Segall and Bradley, 2009].

\section{Isothermal Membrane Diffusion}

[31] A significant simplification occurs when, as observed in some fault zones, the rock adjacent to the shearing zone has an extremely low permeability, whereas rocks farther away are highly fractured and orders of magnitude more permeable (see Rice [2006] for summary of field observations). We may approximate this setting with a low-permeability wall zone, of thickness $h_{w}$, bordering the fault surrounded by an external reservoir that is sustained at constant pore pressure $p^{\infty}$. For times that are long compared to the characteristic time for diffusion through the border zone, the solution of the isothermal form of (9) subject to the stated boundary conditions yields $\partial p /\left.\partial y\right|_{y=0}=-\left(p-p^{\infty}\right) / h_{w}$. Applying this to the isothermal form of (10) yields,

$$
\frac{\partial p}{\partial t}=\frac{2 c_{h y d}}{h h_{w}}\left(p^{\infty}-p\right)-\frac{1}{\beta} \frac{\partial \phi}{\partial t}=\frac{p^{\infty}-p}{t_{f}}-\frac{1}{\beta} \frac{\partial \phi}{\partial t}
$$

[Segall and Rice, 1995], which we refer to as isothermal membrane diffusion. Here $t_{f}$ is a characteristic diffusion time, and $p$ is the pore pressure within the shearing zone. While (15) is only valid for times that are long compared to the diffusion time across the bordering low-permeability zone, it offers a significant simplification, over the isothermal forms of (9) and (10), or (11).

\subsection{Isothermal Membrane Diffusion: Dimensional Analysis}

[32] We write the equations for the membrane diffusion approximation in nondimensional form as follows. Taking the time derivative of (6) and making use of (2)

$$
\begin{aligned}
& \frac{\mu}{2 \pi(1-\nu)} \int_{-\infty}^{\infty} \frac{\partial v / \partial \xi}{\xi-x} d \xi-(\sigma-p)\left[a \frac{\dot{v}}{v}+b \frac{\dot{\theta}}{\theta}\right] \\
& +f(\theta, v) \frac{\partial p}{\partial t}=\frac{\mu}{2 v_{s}} \frac{\partial v}{\partial t}
\end{aligned}
$$

The membrane diffusion approximation (15) is combined with the dilatancy law (5), yielding

$$
\frac{\partial p}{\partial t}=\frac{p^{\infty}-p}{t_{f}}+\frac{\epsilon}{\beta} \frac{\dot{\theta}}{\theta}
$$

Equations (16) and (17) combined with the state evolution law (3) define the system. We choose the same nondimensional time as in section 2.1, define $\tilde{v}=v / v^{\infty}$ and $\tilde{f}=$ $f / f_{0}$, and normalize the along-fault distance scale by $h_{d r}^{*}$, where $h_{d r}^{*}$ is the drained critical stiffness from equation (1) with $C=1$,

$$
\tilde{x}=\frac{x}{h_{d r}^{*}}=\frac{x\left(\sigma-p^{\infty}\right)(b-a)}{\mu(1-\nu) d_{c}} .
$$

Assuming the slip law form of the state evolution equations, this leads to the following system of equations

$$
\begin{gathered}
\left(\frac{b-a}{b}\right) \frac{1}{2 \pi} \int_{-\infty}^{\infty} \frac{\partial \tilde{v} / \partial \tilde{\xi}}{\tilde{\xi}-\tilde{x}} d \tilde{\xi}-\frac{(\sigma-p)}{\left(\sigma-p^{\infty}\right)}\left[\frac{a \dot{\tilde{v}}}{b}+\frac{\dot{\tilde{\theta}}}{\tilde{\tilde{v}}}\right]+\frac{f_{0} \tilde{f}(\tilde{\theta}, \tilde{v})}{b\left(\sigma-p^{\infty}\right)} \frac{\partial p}{\partial \tilde{t}} \\
=\frac{\mu}{2 b\left(\sigma-p^{\infty}\right)} \frac{v^{\infty}}{v_{s}} \frac{\partial \tilde{v}}{\partial \tilde{t}} \\
\frac{f_{0}}{b\left(\sigma-p^{\infty}\right)} \frac{\partial p}{\partial \tilde{t}}=\frac{f_{0}}{b} \frac{\left(p^{\infty}-p\right)}{\left(\sigma-p^{\infty}\right)}\left(\frac{d_{c}}{v^{\infty} t_{f}}\right)+\frac{f_{0} \epsilon}{\beta b\left(\sigma-p^{\infty}\right)} \tilde{\tilde{\theta}} \\
\frac{\tilde{\tilde{\theta}}}{\partial \tilde{\theta}}=-\tilde{\theta} \tilde{v} \ln (\tilde{\theta} \tilde{v})
\end{gathered}
$$

The appropriate scaling for both effective stress and pore pressure is the nominal effective stress $\left(\sigma-p^{\infty}\right)$. Equation (20) reveals two dimensionless parameters that are important for understanding the effects of dilatancy on friction. From equation (19) note that the importance of pore pressure induced changes in strength relative to rate-state friction changes in strength is given by $\left(f_{0} / b\right) \partial \tilde{p} / \partial \tilde{t}$. The nondimensional pore pressure change, $\left(f_{0} / b\right) \partial \tilde{p} / \partial \tilde{t}$, is given by $(20)$, which depends on two dimensionless parameters. The first,

$$
\mathcal{E} \equiv \frac{f_{0} \epsilon}{\beta b\left(\sigma-p^{\infty}\right)}
$$

gives the importance of dilatancy relative to frictional weakening. This scaling arises because dilatant strengthening scales with $f_{0} \epsilon / \beta$, whereas frictional weakening scales with $b\left(\sigma-p^{\infty}\right)$. Note importantly that dilatancy is relatively stronger when the effective stress is low. In general, we expect dilatancy to be significant relative to rate and state friction if $\mathcal{E} \gtrsim 1$, assuming that fluid drainage is not so fast that the fault zone pore pressure remains unchanged (drained conditions). Assuming that $b \sim 10^{-2}, f_{0}=0.6, \beta$ is in the range 5 to $10 \times 10^{-11} 1 / \mathrm{Pa}$ [Segall and Rice, 2006; Rice, 2006], and $\epsilon$ is in the range of $10^{-5}$ to $10^{-4}$, then $\mathcal{E}=1$ occurs for effective normal stresses of from 4 to $100 \mathrm{MPa}$. This suggests that, especially at low effective stresses, dilatancy will be significant in controlling fault strength. 


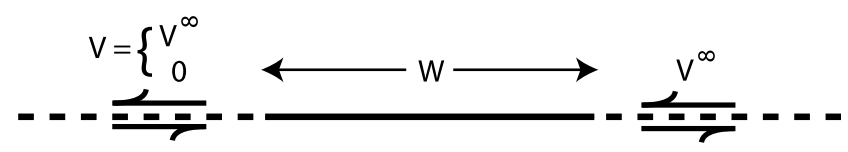

Figure 3. $W$ is the width of the zone over which rate and state friction and dilatancy are computed. At the right boundary the slip rate is set to $v^{\infty}$ ("downdip"), whereas the left boundary is set either to $v^{\infty}$ or for all results presented here to $10^{-3} v^{\infty}$ ("updip").

[33] The fluid transport term in (20) (first term on right) scales with

$$
\mathcal{U} \equiv \frac{v^{\infty} t_{f}}{d_{c}}
$$

the ratio of the characteristic fluid diffusion time to the characteristic time for state evolution. In response to changes in state near a slip speed of $v^{\infty}$, the system is effectively undrained when $\mathcal{U} \gg 1$ and drained when $\mathcal{U} \ll 1$. In the limit of drained behavior the system behaves as in the absence of dilatancy. For $\mathcal{U}<1$ we expect the system to transition to undrained behavior for slip speeds $v / v^{\infty} \geq \mathcal{U}^{-1}$.

[34] The system is completely described by the following dimensionless parameters: $\mathcal{E}, \mathcal{U}, a / b, W / h_{d r}^{*}, f_{0} / b$ (which is of order 30 based on laboratory data), and $\mu v^{\infty} / 2 b\left(\sigma-p^{\infty}\right) v_{s}$. The last quantity scales the radiation damping term; because $v^{\infty} / v_{s} \ll 1$ this term is insignificant until the nondimensional accelerations become of order $10^{8}$.

[35] The system of equations (19), (20), and (21), are solved assuming periodic boundary conditions in the alongfault, $x$ direction. This allows the convolution term in (16) associated with elastic stress interactions to be computed in the Fourier domain. The width of the fault for which friction and dilatancy are computed is $W$ (Figure 3). Outside this region constant slip rate equal to the plate velocity $v^{\infty}$ is imposed on both edges (symmetric loading), or on one side with the other side set to $10^{-3} v^{\infty}$ (asymmetric loading), to roughly approximate a frictionally locked interface. To properly resolve the propagating front of the slow slip zone we require the spatial grid in the along-fault direction to be on the order of $1 / 20$ of the length scale $L_{d} \equiv(1-a / b) h_{d r}^{*}$. Although in general this is too coarse for the slip law alone [Ampuero and Rubin, 2008], dilatancy spreads out the front to the extent that it is sufficient for our purposes. The governing equations (16), (17), and (21) are cast as a coupled system of first-order ordinary differential equations in $d v / d t$, $d \theta / d t$, and $d p / d t$ that are integrated using ODE solvers in Matlab. Because state decreases with increasing slip speed, we have found it to be useful to map the parameters $v$ and $\theta$ to the variables $\ln \left(v / v_{0}\right)$ and $\ln \left(v \theta / d_{c}\right)$.
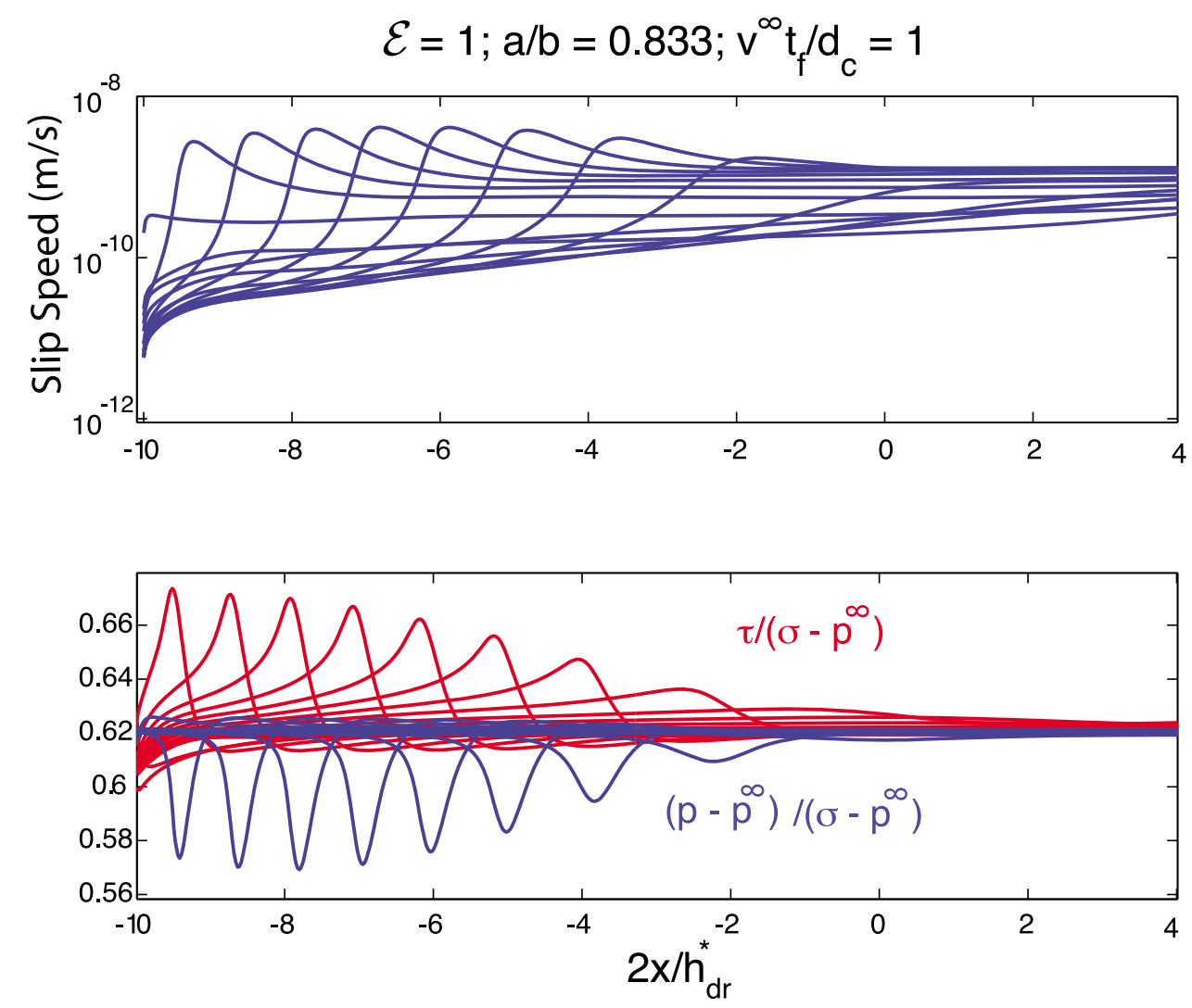

Figure 4. A representative calculation with asymmetric loading. Each curve represents a snapshot, not evenly spaced, in time. Slip event propagates from right to left. (top) Slip speed. (bottom) Shear stress and pore pressure change, both normalized by nominal effective stress. Pore pressure curves are offset vertically by the nominal friction; far from the rupture front $p-p^{\infty}=0$. Isothermal, membrane diffusion approximation. 
(a) $\mathcal{E}=0.32 ; a / b=0.7 ; v^{\infty} t_{f} / d_{c}=0.1 ; W / h^{*}=8$

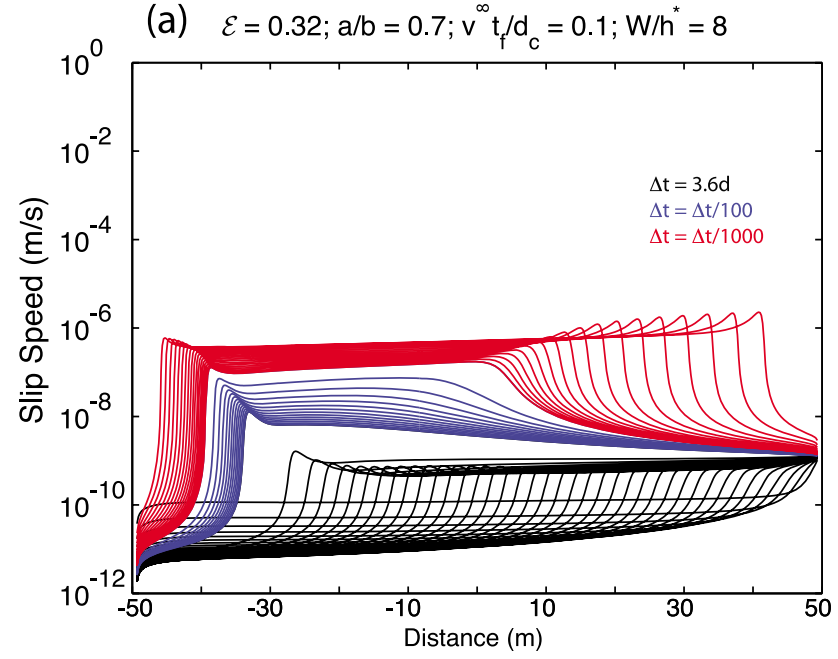

(b) $\mathcal{E}=0.25 ; \mathrm{a} / \mathrm{b}=0.7 ; \mathrm{v}^{\infty} \mathrm{t}_{\mathrm{f}} / \mathrm{d}_{\mathrm{c}}=0.1 ; \mathrm{W} / \mathrm{h}^{*}=8$

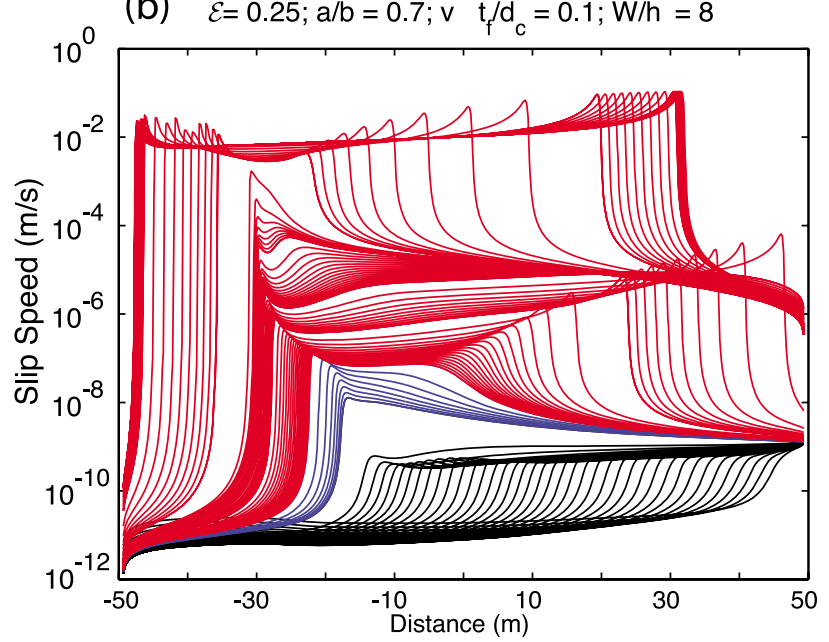

Figure 5. Variation in behavior with changes in $\mathcal{E}$ : (a) $\mathcal{E}=0.32$ and (b) $\mathcal{E}=0.25$. All other parameters are the same in both calculations, $\mathcal{U}=0.1, W / h_{d r}^{*}=8$, and $a / b=0.7$. Isothermal, membrane diffusion. Different colors in Figure 5a indicate different (nonuniform) time intervals between snapshots. In Figure $5 \mathrm{~b}$ the time steps continue to decrease by factors of 10 (gaps in the snapshots) as the slip speed increases.

\subsection{Membrane Diffusion Results}

[36] Figure 4 shows a sample calculation with $\mathcal{E}=1$, $\mathcal{U}=1, a / b=0.833, W / h_{d r}^{*}=8$, and the slip law. Slip propagates from the right to left driven by the increasing displacement at the right boundary. The maximum slip rate increases as the slip event expands, but remains less than an order of magnitude above $v^{\infty}$. In these calculations $v^{\infty}$ is taken to be $0.04 \mathrm{~m} / \mathrm{yr} \simeq 1.3 \times 10^{-9} \mathrm{~m} / \mathrm{s}$. Figure 4 also shows a significant stress concentration as well as pore pressure reduction at the front of the propagating slip front. It is the relative suction at the rupture front that stabilizes the slip against dynamic instability. This can be seen by comparing with Figure 1, which shows that with the slip law for $a / b=$ 0.8 and no dilatancy, slip becomes dynamic for $W / h_{d r}^{*} \sim 3$. For $a / b=0.833$ we expect that the transition to inertially limited slip to occur at only a slightly larger value of $W / h_{d r}^{*}$. In contrast, with membrane diffusion dilatancy and $\mathcal{E}=0.6$, $\mathcal{U}=1$, Figure 1 shows that slip is stable to at least $W / h_{d r}^{*}=48$. These results demonstrate that dilatancy is capable of stabilizing slip over a broad range of fault lengths.

[37] Figure 5 compares two solutions with slightly different values of $\mathcal{E}$. In both cases $\mathcal{U}=0.1, W / h_{d r}^{*}=8$, and $a / b=$ 0.7 . In one $\mathcal{E}=0.32$, whereas in the other it is reduced slightly to $\mathcal{E}=0.25$. For $\mathcal{E}=0.32$, a slip event propagates from the right-hand (constant slip rate) boundary with maximum slip rates on the order of the plate velocity. At some point, before the rupture front reaches the "locked" boundary, slip accelerates to order of $10^{-6} \mathrm{~m} / \mathrm{s}$, and begins to propagate bilaterally (Figure 5a). The slip rate, however, is always well within the quasi-static regime. Reducing $\mathcal{E}$ only slightly to 0.25 , changes the behavior dramatically. The left-propagating front accelerates, spawning a fast rightward propagating phase that dies out after encountering the fixed velocity boundary (Figure $5 \mathrm{~b}$ ). The left-propagating front continues to accelerate, eventually spawning a second rightward propagating phase. Ultimately, slip reaches order $0.1 \mathrm{~m} / \mathrm{s}$ at which point radiation damping effects are significant, and the rupture is considered dynamic. In section 3.3 we show that a linearized stability analysis can provide guidance in explaining the difference in behavior for relatively small changes in $\mathcal{E}$.

\subsection{Membrane Diffusion: Linearized Stability Analysis}

[38] Segall and Rice [1995] conducted a linearized stability analysis for the membrane diffusion model with spring slider elasticity. They find that, as in the drained case, a critical spring stiffness $k_{c r i t}$ exists such that small perturbations from steady state are damped for stiffness greater than $k_{c r i t}$, but grow without bound when the stiffness is less than this critical value. The critical stiffness is given by

$$
k_{\text {crit }}=\left(\sigma-p^{\infty}\right) \frac{(b-a)}{d_{c}}-\frac{f_{0} \varepsilon}{\beta d_{c}} F(\mathcal{E}, \mathcal{U}, a / b)
$$

where the function $F(\mathcal{E}, \mathcal{U}, a / b)$ is

$$
\begin{gathered}
F=\left[\frac{1+\lambda+\gamma}{2}-\sqrt{\frac{(1+\lambda+\gamma)^{2}}{4}-\gamma}\right] \\
\lambda=\mathcal{E}^{-1}\left(\frac{a}{b}\right) \quad \frac{1}{\mathcal{U}+\mathcal{U}^{2}} \\
\gamma=\mathcal{E}^{-1}\left(\frac{b-a}{b}\right) \frac{\mathcal{U}}{\mathcal{U}+1} .
\end{gathered}
$$

The limiting behavior for fast drainage is $F(\mathcal{U} \rightarrow 0)=0$, so that the drained stiffness is equivalent to that found by Ruina [1983]. Normalizing the critical stiffness by the drained critical stiffness, we have

$$
\tilde{K}_{c r i t}=\frac{k_{c r i t} d_{c}}{\left(\sigma-p^{\infty}\right)(b-a)}=1-\mathcal{E}\left(\frac{b}{b-a}\right) F(\mathcal{E}, \mathcal{U}, a / b) .
$$



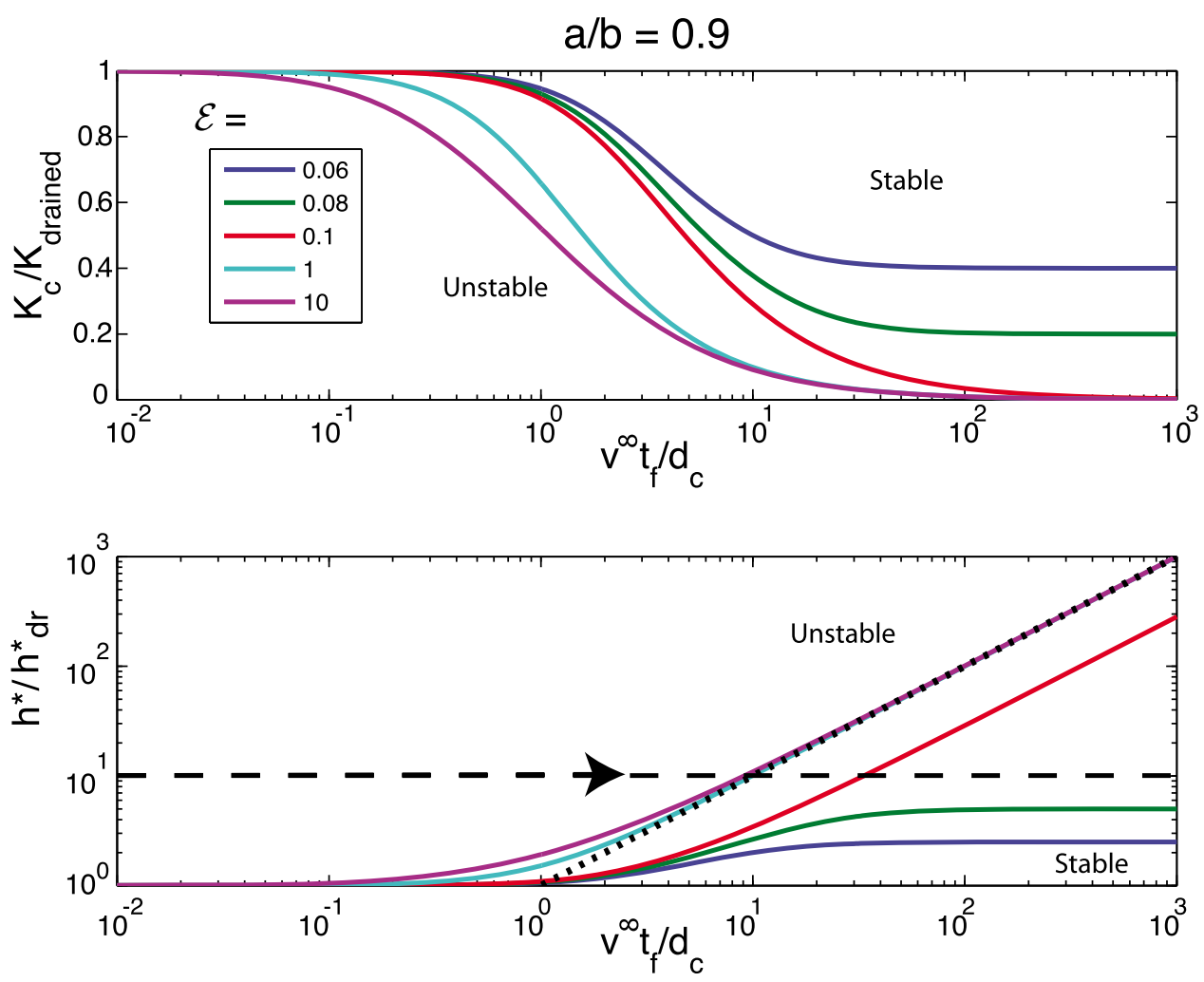

Figure 6. Stability boundary for membrane diffusion model for $a / b=0.9$. (top) Normalized critical stiffness. System is linearly stable for stiffness less than the critical value (below the curve for given value of $\mathcal{E}$ ). (bottom) Normalized critical crack length $h^{*} / h_{d r}^{*}$. System is stable for lengths less than the critical length. Dotted line indicates asymptotic undrained limit given by equation (31). Dashed line indicates hypothetical path.

In the undrained limit, $\mathcal{U} \rightarrow \infty, \lambda \rightarrow 0$, and $\tilde{K}_{\text {crit }}$ exhibits two limiting behaviors. For $\gamma>1, \lim _{u \rightarrow \infty} F=1$ and

$$
\tilde{K}_{\text {crit }}^{\mathcal{U}=\infty}=1-\mathcal{E}\left(\frac{b}{b-a}\right)
$$

If, on the other hand, $\gamma \leq 1$ then, $\lim _{\mathcal{U} \rightarrow \infty} F=\gamma$. This implies that in the undrained limit, with $\gamma \leq 1$, that $\tilde{K}_{\text {crit }}^{\mathcal{u}=\infty}=0$. Since the stiffness is nonnegative, the system is linearly stable in the undrained limit for all stiffnesses if $\gamma \leq 1$. For $\mathcal{U} \gg 1$ the condition that $\gamma \leq 1$ corresponds to

$$
\mathcal{E}_{\text {crit }} \geq 1-\frac{a}{b}
$$

This result was obtained earlier by Segall and Rice [1995, equation (28)] in the context of a critical pore pressure above which instabilities are suppressed. [Note that if we had defined $\mathcal{E}$ by $\mathcal{E}=f_{0} \epsilon / \beta(b-a)\left(\sigma-p^{\infty}\right)$, then the condition (30) would be $\left.\mathcal{E}_{\text {crit }} \geq 1\right]$.

[39] Figure 6 illustrates the linearized stability boundary for $a / b=0.9$ as a function of $\mathcal{U}=v^{\infty} t_{f} / d_{c}$ and different values of $\mathcal{E}$. For $a / b=0.9, \mathcal{E}_{\text {crit }}=0.1$, from (30). For a fixed value of $\mathcal{E}$ the critical stiffness decreases with increasing $\mathcal{U}$, as pore fluid flow is less and less able to compensate for dilatancy. For $\mathcal{E}<\mathcal{E}_{\text {crit }}$ the undrained critical stiffness is positive and for sufficiently low stiffness the system is lin- early unstable. On the other hand, for $\mathcal{E} \geq \mathcal{E}_{\text {crit }}$ the undrained stiffness is zero and the system is stable for all stiffnesses.

[40] The predicted critical crack length is inversely proportional to the critical stiffness. Thus, $h^{*} / h_{d r}^{*}$ is equal to the inverse of the normalized critical stiffness, $\tilde{K}$. It can be shown from (27) that for $\gamma \ll 1$, the limiting behavior as $\mathcal{U} \rightarrow \infty$ is $\tilde{K}_{\text {crit }}^{\mathcal{U}=\infty}=1 / \mathcal{U}$, so that in this same limit

$$
\lim _{\mathcal{U} \rightarrow \infty} \frac{h^{*}}{h_{d r}^{*}}=\frac{v^{\infty} t_{f}}{d_{c}} .
$$

This behavior is shown in Figure 6 (bottom), where each curve represents $h^{*} / h_{d r}^{*}$ for a different value of $\mathcal{E}$. For $\mathcal{E}<$ $\mathcal{E}_{c r i t}, h^{*} / h_{d r}^{*}$ asymptotes to a finite value in the undrained limit. In this case a sufficiently long slipping zone will be linearly unstable regardless of $\mathcal{U}$. On the other hand, if $\mathcal{E}>$ $\mathcal{E}_{\text {crit }}, h^{*} / h_{d r}^{*}$ increases linearly with $\mathcal{U}$, such that in the limit $\mathcal{U} \rightarrow \infty$ no slipping zone is long enough to be linearly unstable. The limiting behavior given by equation (31), shown as the dotted line in Figure 6, well approximates the behavior for $\mathcal{E} \gg \mathcal{E}_{\text {crit }}$ and $\mathcal{U} \gg 1$.

\subsection{Application of Linear Stability Results}

[41] The above result applies to small perturbations from steady sliding at speed $v^{\infty}$; however, it may provide insight more generally into the tendency for slip to accelerate in the following sense. Consider slip at rate $v$ that is nearly uniform spatially. Local acceleration from this quasi-uniform slip 


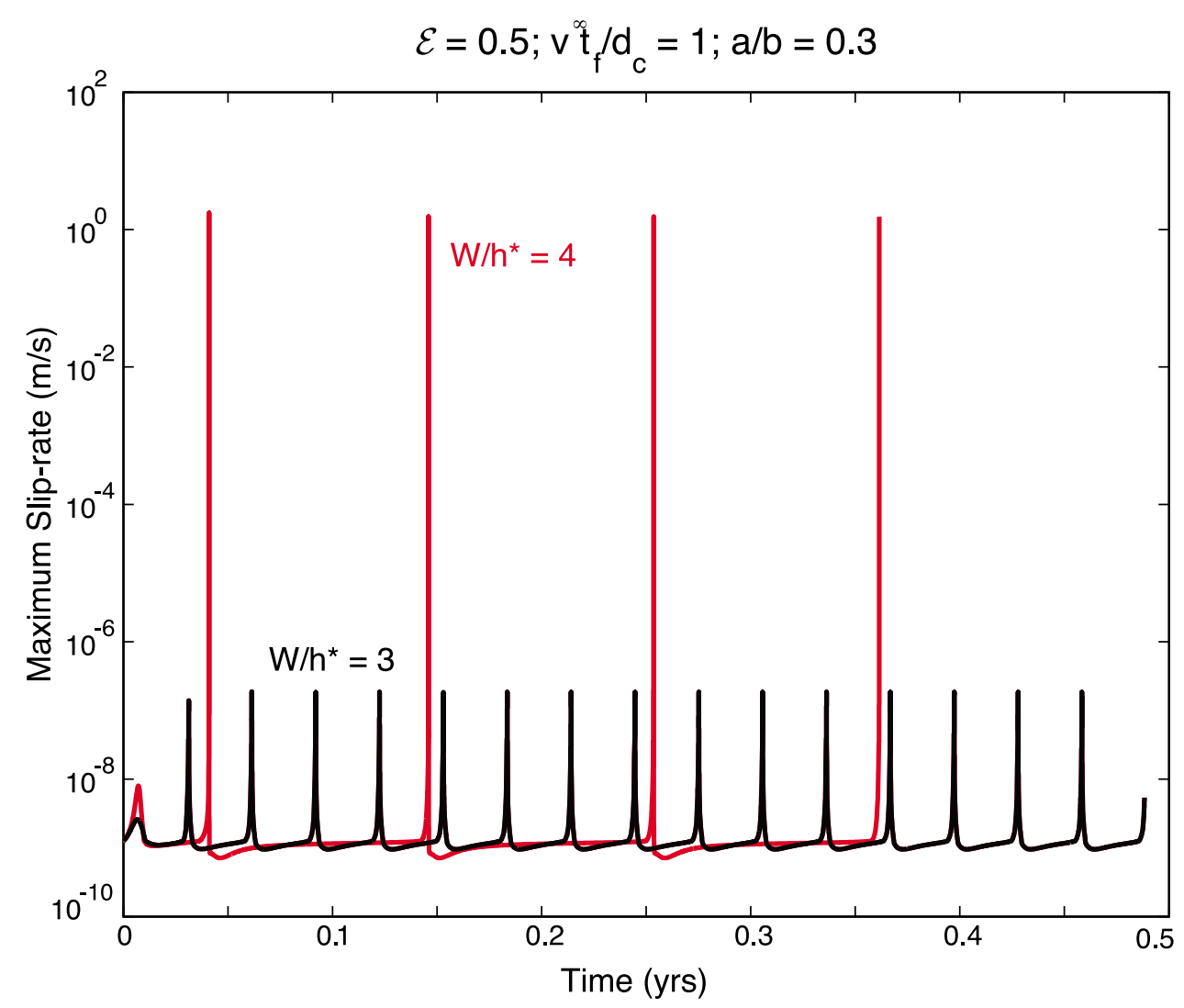

Figure 7. Maximum slip rate for $\mathcal{E}=0.5, \mathcal{U}=1$, and $a / b=0.3$ for two different values of $W / h_{d r}^{*}$. For $W / h_{d r}^{*}=3$ there are periodic slow slip events, while for $W / h_{d r}^{*}=4$ there are periodic dynamic slip events.

could be considered as a perturbation from the locally steady sliding, in which case the previous results hold with $v$ replacing $v^{\infty}$. Dilatancy itself promotes more spatially uniform slip rate distributions than would be obtained under drained conditions; any section of the fault that accelerates above the background slip rate experiences less effective drainage and therefore a greater strengthening effect. This is illustrated in Figure 5, where the tendency for slip speed to increase at the slip front is mitigated by dilatant strengthening, allowing the remainder of the slip zone to "catch up," leading to rather uniform slip rate profiles (at least on a logarithmic scale).

[42] As noted by Liu and Rubin [2010], further insight can be gained by considering the slip to be fast enough that the fault is completely undrained on the time scale of the slow event. Assuming that the fault had previously been slipping at steady state, and that well behind the propagating front of the slow event the friction has reached its steady state value, then the stress drop for a unit increase in $\ln v$ is given by $-d \tau_{s s} / d \ln v$ at constant fluid mass. From Segall and Rice [1995, equation (20)] in current notation, this is $b\left(\sigma-p^{\infty}\right)$ $(1-a / b-\mathcal{E})$, so that the stress drop becomes negative in the undrained limit when $\mathcal{E}>1-a / b$.

[43] These considerations suggest that the linearized stability analysis may provide useful insights even far from steady state conditions. As discussed above, the linearized analysis implies that for $\mathcal{E}<\mathcal{E}_{\text {crit }}$ a sufficiently long slipping zone can become dynamically unstable, whereas for $\mathcal{E}>$ $\mathcal{E}_{\text {crit }}$, any slipping zone, no matter how long, will eventually slip fast enough to become effectively undrained at which point further acceleration is inhibited. This is illustrated in Figure 6 for a hypothetical slipping zone with $W / h_{d r}^{*}=10$, shown by the dashed line. For $\mathcal{E}=0.08$ for example, this zone is sufficiently long that it is predicted to remain in the unstable regime as $v$ increases. On the other hand, if $\mathcal{E}=1$ an accelerating slip zone intersects the stability boundary near $v^{\infty} t_{f} / d_{c} \sim 10$. The conclusion is that for nominally unstable friction and $\mathcal{E} \geq \mathcal{E}_{\text {crit }}$, slip will ultimately be stabilized by dilatancy regardless of the width of the slipping zone, $W / h_{d r}^{*}$. Of course, for sufficiently large $W / h_{d r}^{*}$ the slip speed at which dilatancy is predicted to stabilize against further acceleration may exceed inertial limits.

[44] Figure 7 illustrates simulations for $a / b=0.3$, which according to equation (30) is conditionally stable for $\mathcal{E}<0.7$. For $\mathcal{E}=0.5$, and $\mathcal{U}=1$ the nondimensional critical length, $h^{*} / h_{d r}^{*}$ is predicted to be $\simeq 1.5$, whereas in the undrained limit $h_{u n}^{*} / h_{d r}^{*} \simeq 3.5$. Indeed, for $W / h_{d r}^{*}=3$ the system responds with periodic noninertial slip events with maximum slip speeds of order $10^{-7} \mathrm{~m} / \mathrm{s}$, whereas for $W / h_{d r}^{*}=4$, maximum slip speeds reach the order of $1 \mathrm{~m} / \mathrm{s}$ (Figure 7). Increasing $\mathcal{E}$ to be greater than or equal to $\mathcal{E}_{\text {crit }}$, which for this value of $a / b$ is 0.7 , however, leads to stable slip events that do not reach inertial speeds. For example, Figure $8 \mathrm{a}$ shows the case for $\mathcal{E}=\mathcal{E}_{\text {crit }}=0.7$ for $W / h_{d r}^{*}=12$ and 15 . 
a.
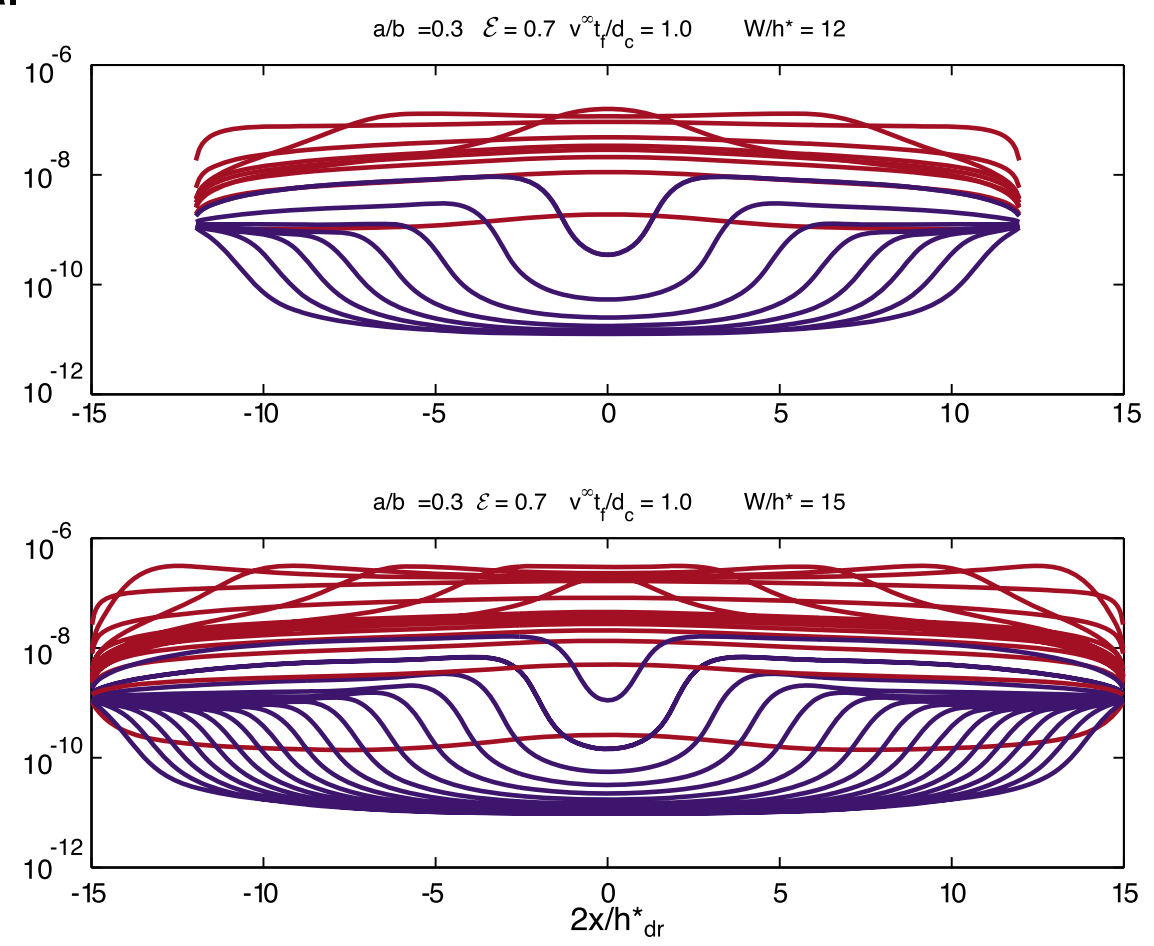

b.
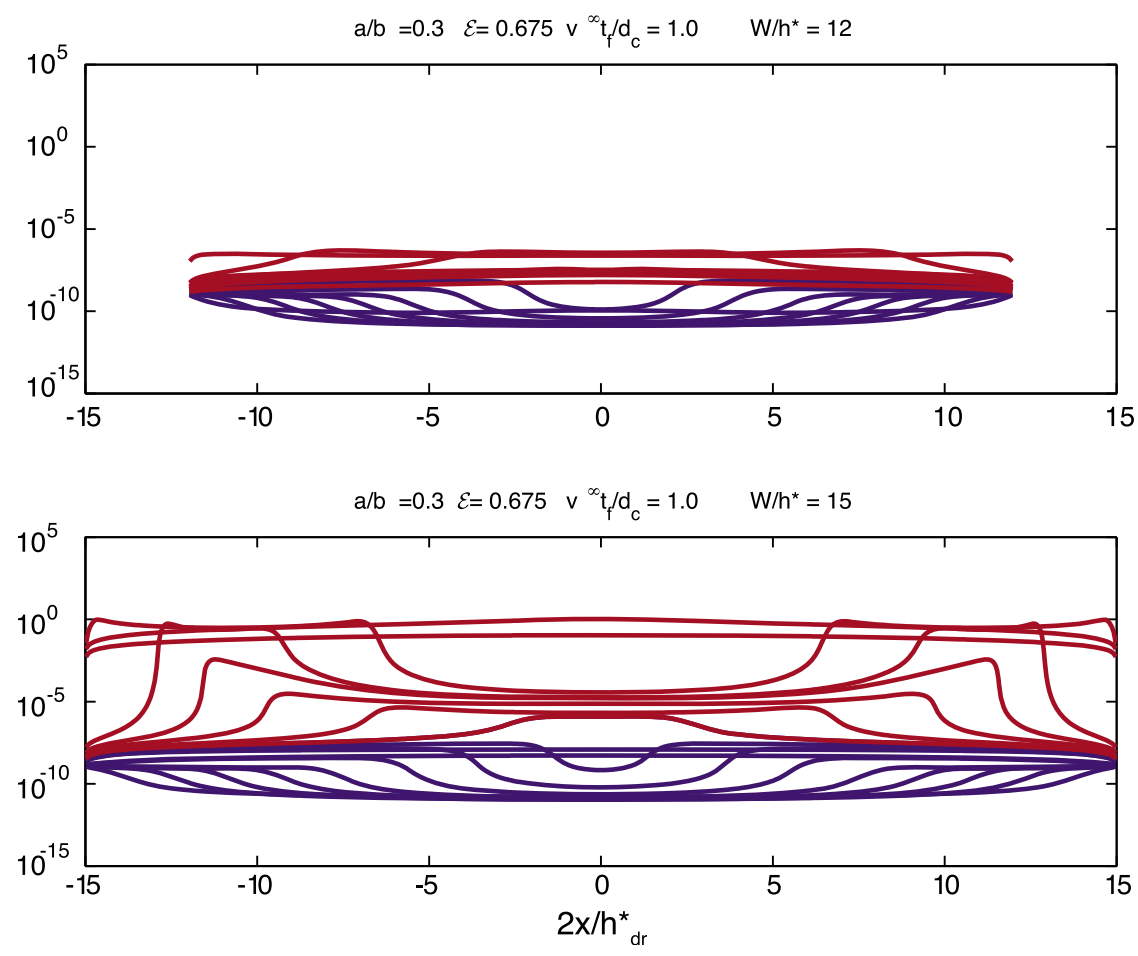

Figure 8. Slip speed as a function of along fault distance, for symmetric loading conditions. Each curve marks a snapshot, not equally spaced, in time. Blue curves denote inward propagating slow phase, whereas red curves mark outward propagating faster slip followed by deceleration. Note different velocity scale in Figure $8 \mathrm{~b}$. (a) $\mathcal{E}=0.7, \mathcal{U}=1$, and $a / b=0.3$ with (top) $W / h_{d r}^{*}=12$ and (bottom) $W / h_{d r}^{*}=15$. (b) $\mathcal{E}=0.675, \mathcal{U}=1$, and $a / b=0.3$ with (top) $W / h_{d r}^{*}=12$ and (bottom) $W / h_{d r}^{*}=15$. 


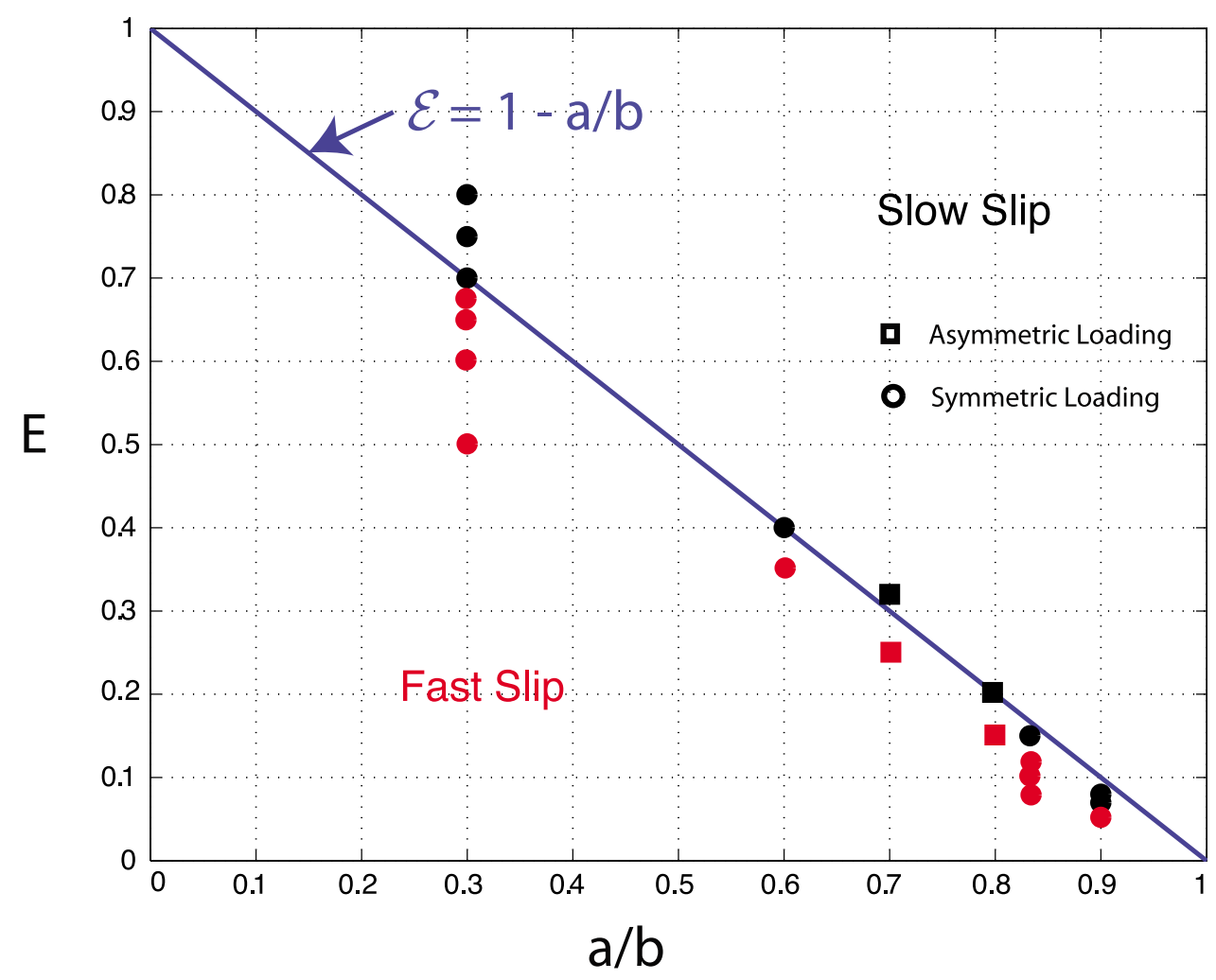

Figure 9. Stability boundary between fast and slow slip. Each symbol may represent multiple runs with varying $W / h_{d r}^{*}$. Red symbols indicate solutions that reach radiation damping velocities for a sufficiently large $W / h_{d r}^{*}$. Black symbols represent solutions that never reach inertially limited slip speeds regardless of $W / h_{d r}^{*}$. Square symbols are loaded from one side, and circles are loaded from both sides. Line $\mathcal{E}=1-a / b$ is the linearized stability boundary between stable and unstable domains.

In both cases slip is stable, with repeated cycles of first a slow inward propagating phase, shown in blue, followed by a faster outward propagating phase, shown in red. Decreasing $\mathcal{E}$ from 0.7 to 0.675 , however, leads to the potential for unstable slip, as shown in Figure $8 \mathrm{~b}$, for the two same values of $W / h_{d r}^{*}$. For $W / h_{d r}^{*}=12 \mathrm{slip}$ is stable and the behavior is similar to that observed for $\mathcal{E}=0.7$ (Figure 8). However, for $W / h_{d r}^{*}=15$ the outward propagating phase accelerates to radiation damping limits. This is consistent with the prediction that $\mathcal{E}=\mathcal{E}_{\text {crit }}$ marks a qualitative boundary in behavior. Note that for $\mathcal{E}=0.675$, the stability analysis predicts that in the undrained limit $h_{u n}^{*} / h_{d r}^{*} \simeq 28$, roughly a factor of 2 greater than the length at the observed transition to unstable slip. When the two inward propagating phases meet in the center a localized region of fast slip initiates. This presumably allows the transition to dynamic slip to occur at smaller $W / h_{d r}^{*}$ than predicted by the linearized analysis.

[45] The above analysis was repeated for different values of $a / b$ and $\mathcal{E}$, recording the maximum slip rates achieved in each simulation. Maximum slip speeds were recorded many cycles after the onset of the calculation to ensure that the values are not strongly dependent on the initial conditions. Results are shown in Figure 9. If the maximum slip speed reaches dynamic values (taken to be $0.1 \mathrm{~m} / \mathrm{s}$ ) for a sufficiently large steady state weakening zone $W / h_{d r}^{*}$ (a wide range around the predicted critical value is tested) the result is indicated in red. If, however, the behavior is quasi-static regardless of $W / h_{d r}^{*}$ the result is indicated in black. Values of $W / h_{d r}^{*}$ up to twice the critical value for the largest unstable value of $\mathcal{E}$ were examined; $\mathcal{U}$ is generally within an order of magnitude of 1.0 . The line $\mathcal{E}=1-a / b$ does a remarkably good job of dividing the space into fast and slow slip behavior. The numerically inferred boundary lies slightly below the line $\mathcal{E}=1-a / b$, at least for large $a / b$ where the friction alone is nearly velocity neutral. Note that the simulations shown in Figure 5 are indicated by the two points at $a / b=0.7$; for $\mathcal{E}=0.32$ the maximum slip speed was well below inertial limits, regardless of the length tested, whereas the solution for $\mathcal{E}=0.25$ exhibited fast slip at $W / h_{d r}^{*}=8$.

[46] Another example is shown for asymmetric loading and $a / b=0.7$ in Figure 10 . The conditions are similar to those in Figure 5, except that $\mathcal{U}=1$, rather than 0.1 . Figures $10 \mathrm{a}$ and $10 \mathrm{~b}$ are for $\mathcal{E}=0.25$, which is predicted to be conditionally unstable. Indeed, for $W / h_{d r}^{*}=6$ and $W / h_{d r}^{*}=8$ the maximum slip speed is well below radiation damping limits. For $W / h_{d r}^{*}=6$, the slow phase propagates across the velocity weakening region before the faster phase begins. For $W / h_{d r}^{*}=8$ the fast phase begins before the slow phase has propagated fully across the velocity weakening region. For a slightly longer velocity weakening region, $W / h_{d r}^{*}=10$, the rupture reaches radiation damping limits (not shown). 

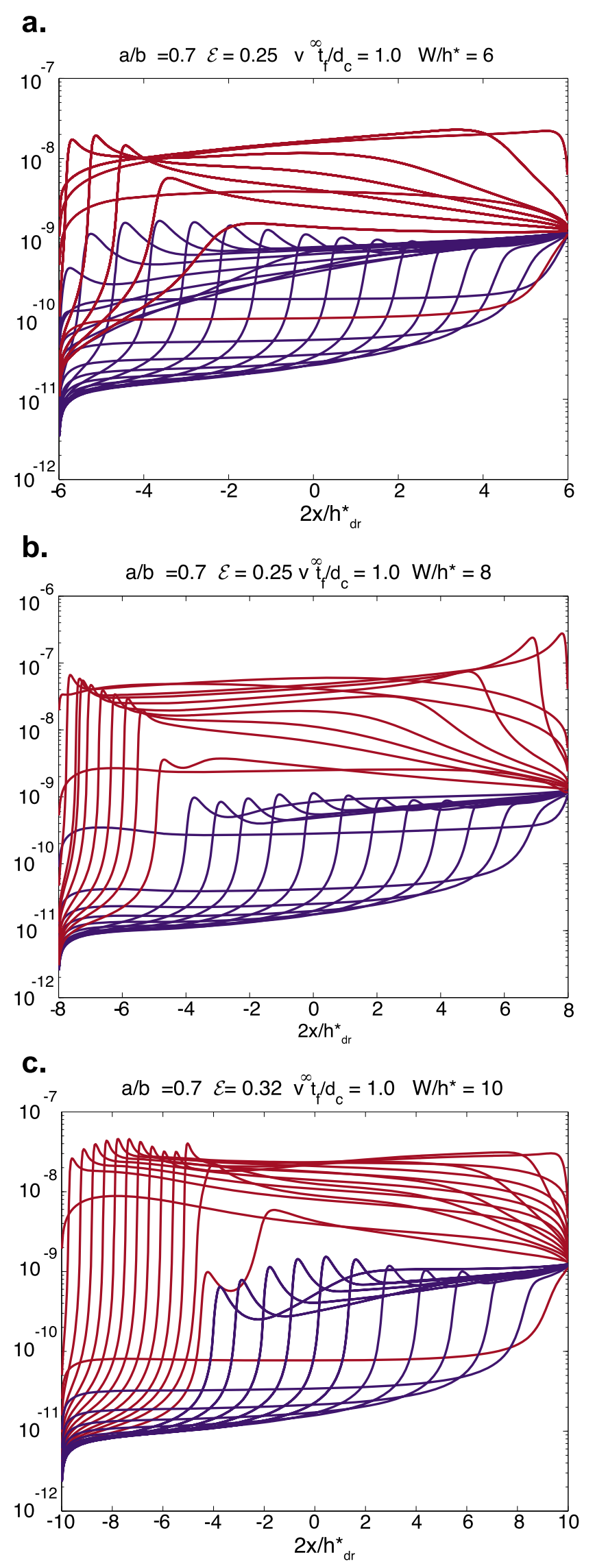

For $\mathcal{U}=0.1$, and all other parameters equal, the transition to unstable behavior occurs at $W / h_{d r}^{*}=8$; see Figure 5 . (The linear stability analysis predicts that the undrained critical width $h^{*} / h_{d r}^{*} \simeq 6$; apparently the fixed boundary condition on the updip end allows the fault width to slightly exceed this critical dimension.) In contrast, if $\mathcal{E}=0.32$, slightly in excess of the critical value of 0.3 , the maximum slip speed for $W / h_{d r}^{*}=10$ is less than $10^{-7} \mathrm{~m} / \mathrm{s}$, as shown in Figure 10c.

[47] Indeed, if the length of the slipping zone is extended by a factor of roughly 2 , to $W / h_{d r}^{*}=18$, with $\mathcal{E}$ maintained at 0.32 , the slip remains quasi-static. The behavior does, however, become significantly more complex. It does remain quasiperiodic, with a period of roughly 0.4 years. Slip speeds averaged over the full velocity weakening region only slightly exceed $10^{-7} \mathrm{~m} / \mathrm{s}$ (Figure 11a). In detail, however, as shown in Figures 11b and 12, there are multiple pulses of slip, all originating at the right-hand ("downdip") boundary. The first phase propagates slowly updip initiating a fast bilaterally propagating phase at about 1.7 years, that ultimately stalls near $x / h^{*}=-10$ (Figures $11 \mathrm{~b}$ and 12 (top left)). Meanwhile, a second pulse starts at the right-hand boundary and propagates updip (Figures $11 \mathrm{~b}$ and 12 (top right)). When this phase reaches roughly $x / h^{*}=-5$, a third phase initiates and propagates rapidly updip (Figure 12, bottom left), eventually overtaking the previous slip rate maxima (Figure 12, bottom right). When the slip rate maxima collide, a much faster phase initiates, propagating bilaterally but most rapidly in the downdip direction (Figures 12 (bottom right) and 11). Ultimately, a similar, but not identical cycle repeats in these simulations. These results emphasize that while stable for $\mathcal{E}>1-a / b$, large values of $W / h_{d r}^{*}$ results in complex behavior with multiple slip events propagating away from the fixed velocity boundary in between periods of high moment release.

[48] There may be cases where the system is nominally unstable in the undrained limit; however, the requisite $W / h_{d r}^{*}$ is extremely large. Reference to Figure 6, however, suggests that this set of conditions may be very small. For $\mathcal{E}=0.08$, which is only $20 \%$ less than $\mathcal{E}_{\text {crit }}$, the undrained critical nucleation dimension is only $\sim 5$ times the drained value. Thus, for $h_{u n}^{*}$ to significantly exceed $h_{d r}^{*}, \mathcal{E}$ would need to be very nearly $\mathcal{E}_{\text {criti }}$, such that $\mathcal{E}=1-a / b$ remains the effective stability boundary.

[49] With reference to Figure 6 one might speculate that for $\mathcal{E}>\mathcal{E}_{\text {crit }}$, the fault accelerates until it reaches the stability boundary. In other words we might associate $v_{\max }$ with $v^{\infty}$ in equation (31), such that

$$
\frac{v_{\max }}{v_{s s}}=\frac{W}{h_{d r}^{*}} \mathcal{U}^{-1},
$$

where $v_{s s}$ is the steady state velocity. This predicts that the maximum velocity increases linearly in $W / h_{d r}^{*}$, is independent

Figure 10. Slip rate as a function of along-fault distance for asymmetric loading, $a / b=0.7$, and $\mathcal{U}=1$. with (a) $\mathcal{E}=0.25$ and $W / h_{d r}^{*}=6$, (b) $\mathcal{E}=0.25$ and $W / h_{d r}^{*}=8$, and (c) $\mathcal{E}=0.32$ and $W / h_{d r}^{*}=10$. Each curve marks a snapshot in time, not equally spaced in time. Blue curves denote the slower phase that propagates away from the boundary fixed at $v^{\infty}$, whereas red curves mark the bilaterally propagating faster phase. 

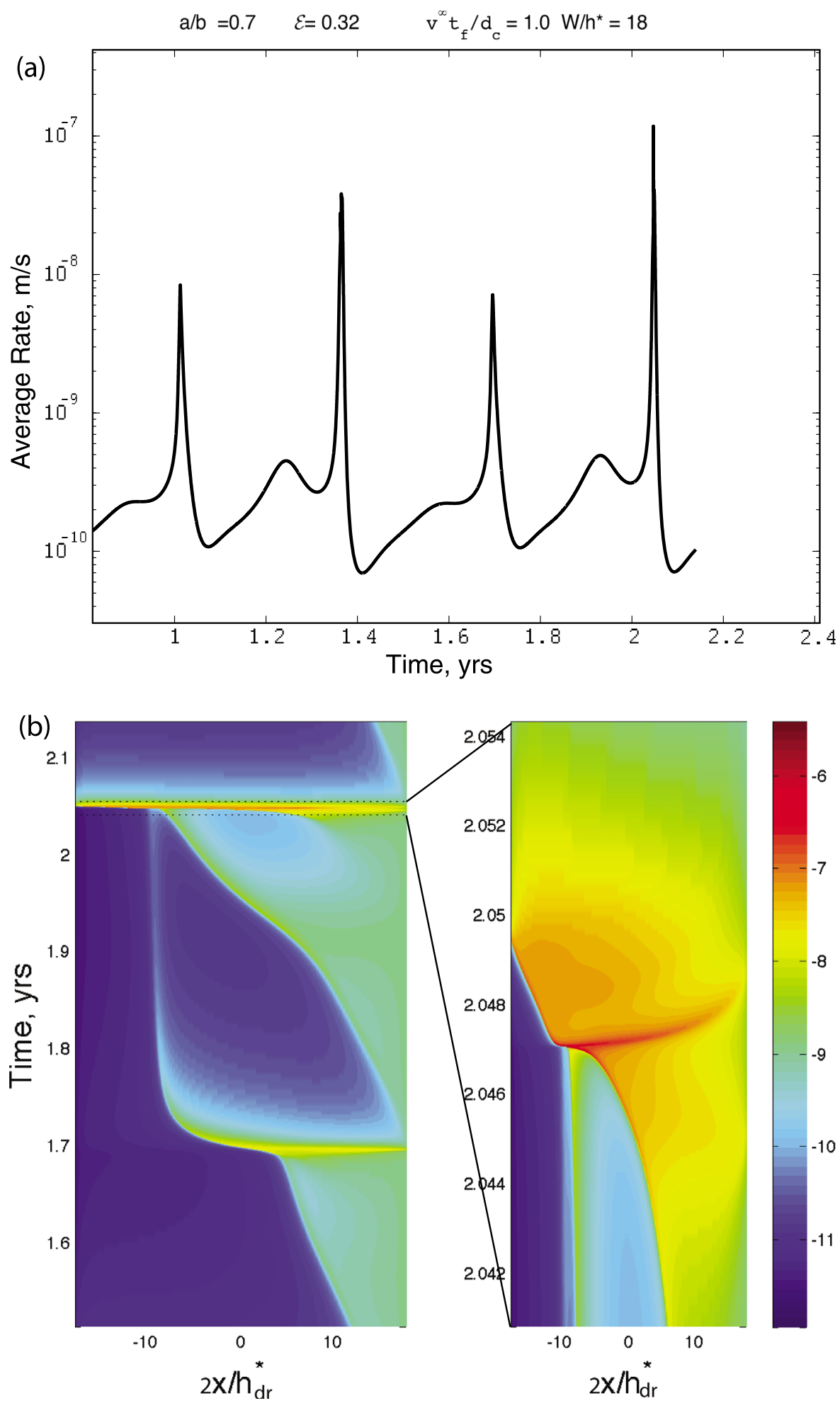

Figure 11. (a) Average slip speed when $a / b=0.7, \mathcal{E}=0.32, \mathcal{U}=1$, and $W / h_{d r}^{*}=18$. (b) Space-time plot showing the evolution of $\log _{10}(v)$, for the last cycle shown in Figure 11a. Colors indicate $\log _{10}$ of the slip speed.

of $\mathcal{E}$, and $a / b$, as long as $\mathcal{E}>1-a / b$. Note that this would be expected to hold only if the velocity distribution is relatively uniform and the friction is near steady state. A limited number of computations does suggest that $v_{\max }$ increases linearly with $W / h_{d r}^{*}$, at least for some range of parameters. Liu and Rubin [2010] estimate the maximum slip speed by approximating the rupture front as a step change in slip rate. Assuming that the interior of the slip zone is 


$$
\mathrm{a} / \mathrm{b}=0.7, \mathcal{E}=0.32, \quad \mathrm{v}^{\infty} \mathrm{t}_{\mathrm{f}} / \mathrm{d}_{\mathrm{c}}=1, \mathrm{~W} / \mathrm{h}^{*}=18
$$
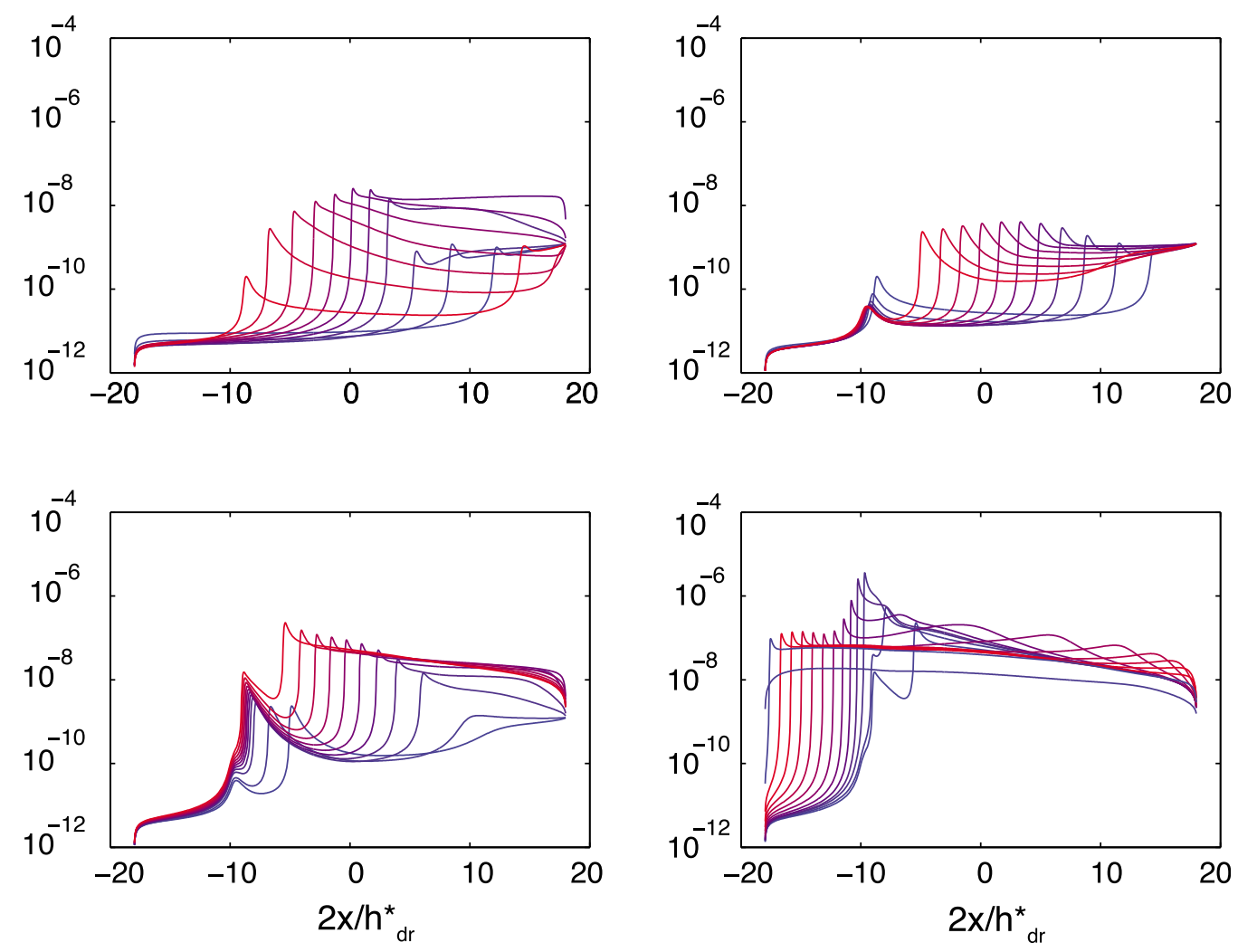

Figure 12. Snapshots of slip speed as a function of along strike distance, $x / h_{d r}^{*}$ at different times, not equally spaced, through a slow slip cycle. In each plot the snapshots grade in color from blue toward red with increasing time. Time goes in the order of top left, top right, bottom left, and bottom right. $a / b=$ $0.7, \mathcal{E}=0.32, \mathcal{U}=1$, and $W / h_{d r}^{*}=18$.

essentially drained and that the effective fracture energy is dominated by dilatancy (implying $\mathcal{E} \gtrsim 1$ ), an energy balance argument leads to

$$
\frac{v_{\max }}{v_{s s}}\left[\ln \left(\frac{v_{\max }}{v_{s s}}\right)\right]^{-1} \propto \frac{W}{h_{d r}^{*}} \frac{\mathcal{E}_{c r i t}}{\mathcal{E}} \mathcal{U}^{-1}
$$

which is not dissimilar to (32), in that $v_{\max }$ grows only slightly faster than linearly with $W / h_{d r}^{*} \mathcal{U}^{-1}$. Whereas (32) is independent of $\mathcal{E}, v_{\max }$ in equation (33) scales with $\mathcal{E}_{\text {crit }} / \mathcal{E}$. On dimensional grounds we anticipate that for $\mathcal{U} \ll 1$ dilatancy effects will no longer be significant, and that the behavior will revert to drained rate and state friction. From equation (32) we expect that for $W / h_{d r}^{*}$ of order 10 , that in order for maximum slip speeds not to exceed $100 \times v^{\infty}$ that $\mathcal{U}$ should not be less than $10^{-1}$. Similarly, for maximum slip speeds not to exceed radiation damping limits we expect that $\mathcal{U}$ should not be less than $10^{-7}$.

[50] Many numerical solutions exhibit periodic, or quasiperiodic, behavior; for example, Figure 11 exhibits periods near 0.3 years. The linearized stability analysis predicts the period of oscillations at neutral stability as a function of the nondimensional parameters $\mathcal{E}, \mathcal{U}$, and $a / b$. For $\mathcal{E}>1-a / b$ the nondimensional period $T v^{\infty} / d_{c}$ in the undrained limit is

$$
\frac{T v^{\infty}}{d_{c}}=2 \pi \mathcal{U} \sqrt{\frac{\mathcal{E}-(1-a / b)}{(1-a / b)}} \quad \mathcal{U} \gg 1 \text { and } \mathcal{E}>1-a / b
$$

Results for $\mathcal{E} \leq 1-a / b$ are given in Appendix A. For reference, the drained result is $T_{d r} v^{\infty} / d_{c}=2 \pi \sqrt{a /(b-a)}$ [Ruina, 1983]. For $\mathcal{U} \gg 1$ the period can be considerably longer than the drained period.

[51] Rubin [2008] found, excluding dilatancy, that for a variety of loading conditions the period normalized by the drained value at neutral stability $T_{d r}$ increased systematically with $W / h^{*}$. Figure 13 shows that the membrane diffusion results, when normalized by $h^{*}$ and $T$ rather than by $h_{d r}^{*}$ and $T_{d r}$, follow the same trend. Figures 13a and 13b show (as dots) the sampled values of $\mathcal{E}$ and $\mathcal{U}$ with $a / b=0.8$ and $W / h_{d r}^{*}=3,6,12$, and (in one case) 24. Superimposed on these are contours showing the values of $T$ and $h^{*}$ accounting for dilatancy (to estimate $T$ for a continuum fault, rather than a spring slider, we must modify equation (A2) in 


\section{$\log \left(T / T_{d r}-1\right)$}
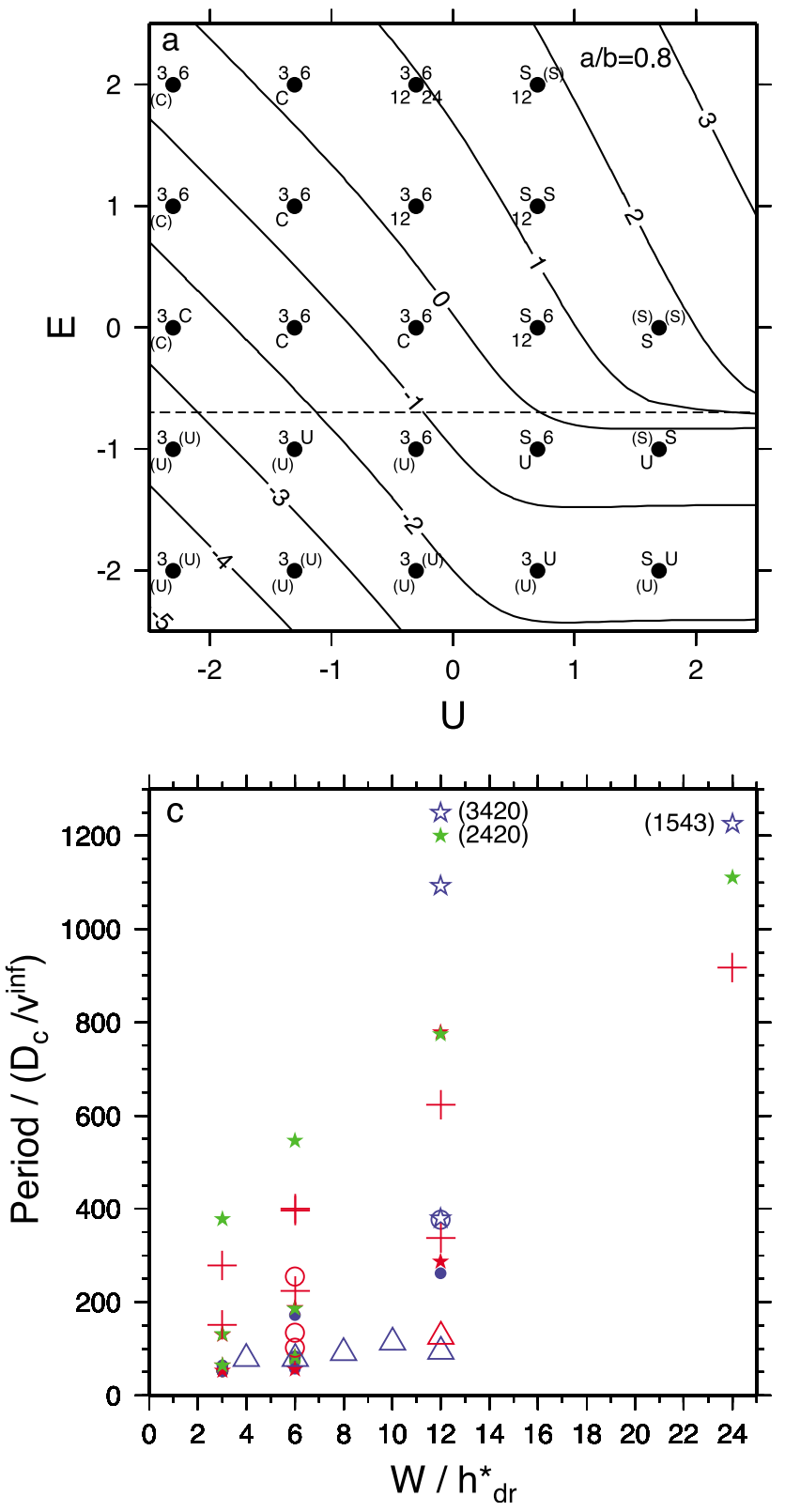

$\log \left(h^{*} / h^{*}{ }_{d r}-1\right)$
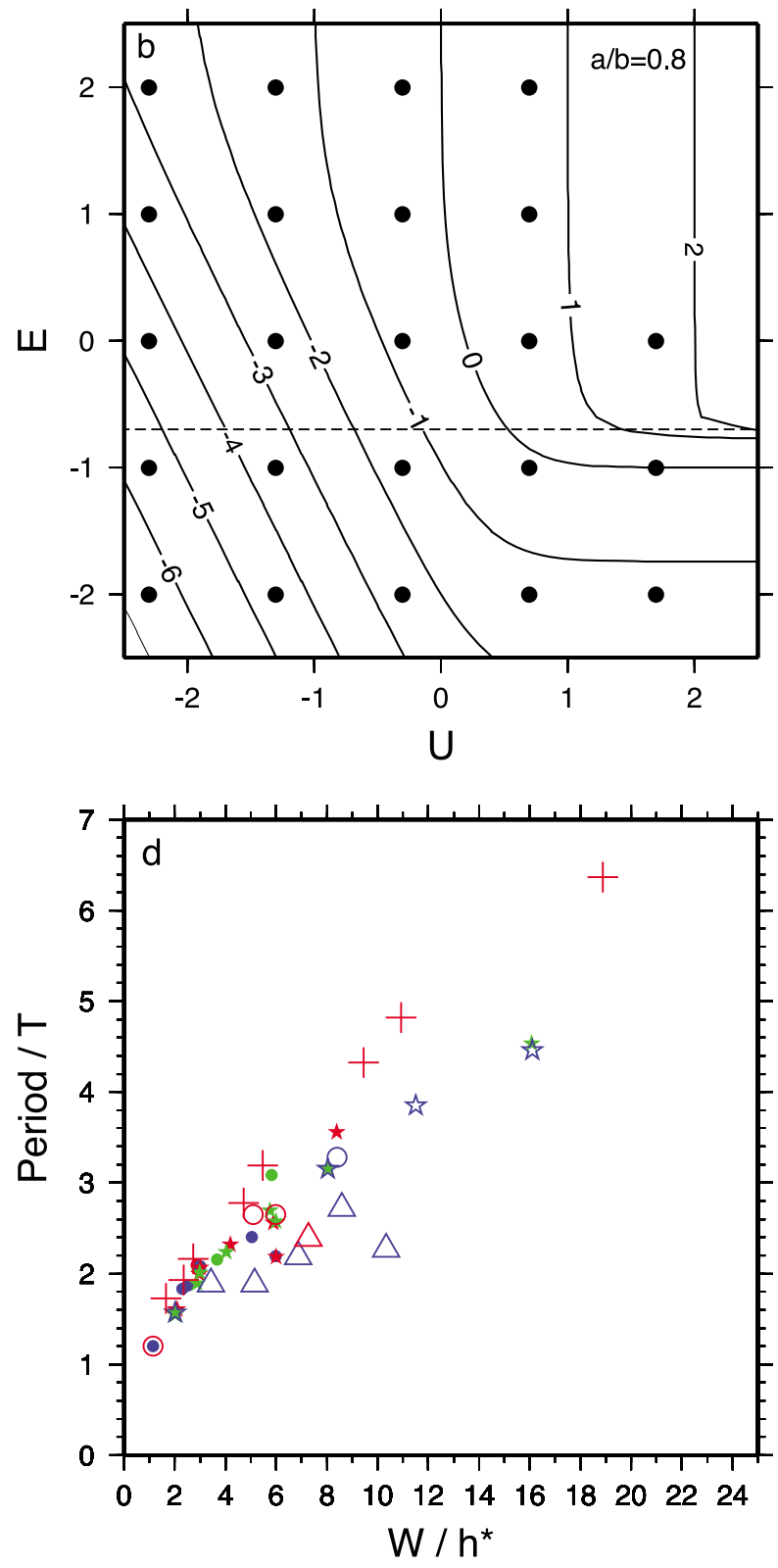

Figure 13. (a and b) Contours of $\ln \left(T / T_{d r}-1\right.$ ) (from equation (A2)) and $\ln \left(h^{*} / h_{d r}^{*}-1\right.$ ) (from the reciprocal of equation (28)) as functions of $\mathcal{E}$ and $\mathcal{U}$, for membrane diffusion simulations with $a / b=0.8$, where $T$ is the period at neutral stability and the subscript " $\mathrm{dr}$ " refers to the drained value. Horizontal dashed line indicates the value of $\mathcal{E}=1-a / b \equiv \mathcal{E}_{\text {crit }}$ separating potentially unstable behavior below from stable behavior above. Dots indicate the values of $\mathcal{E}$ and $\mathcal{U}$ used in numerical simulations with $a / b=0.8$. Numbers $\left(3,6,12\right.$, or 24) indicate values of $W / h_{d r}^{*}$ for which the simulations resulted in periodic or nearly periodic slow slip; results of these are shown in Figures 13c and 13d. Letters in those same locations signify the following: S, stable sliding; C, chaotic velocity excursions; U, unstable slip (speeds limited by radiation damping). Letters in parentheses are inferred from the expectation that more stable sliding is promoted by increasing $\mathcal{E}$, increasing $\mathcal{U}$, and decreasing $W$. (c) Normalized period from periodic or quasiperiodic numerical simulations, as a function of $W / h_{d r}^{*}$. Values of three points that plot off the top of Figure $13 \mathrm{c}$ are indicated in parentheses. Solid symbols are for $a / b=0.8$, open symbols are for $a / b=9$, and different colors and symbols (stars or circles) are for different values of $\mathcal{E}$. Red crosses are for models where $a / b$ increases linearly from 0.8 at the nearly locked end to 1.2 at the forced (downdip) end. Open triangles are for half-space diffusion with $E_{p}=10^{-3}$ (blue) and $3 \times 10^{-3}$ (red). (d) The same data as in Figure 13c but with $W$ now normalized by $h^{*}$ and the period by $T$. 
Appendix A by substituting for $v^{\infty}$ the average slip speed at a representative point within the velocity-weakening region; we take this to be its center, where this slip speed is simply $v^{\infty} / 2$ ). Figure $13 \mathrm{c}$ shows the normalized period for all of these simulations that were either strictly periodic or quasiperiodic. Also included are results from a smaller number of simulations with: $a / b=0.9$ (open circles and stars); (2) a linear gradient in $a / b$ from 0.8 to 1.2 , so that only half the frictional domain was velocity-weakening (red crosses); and (3) homogeneous half-space diffusion (see section 4) with a uniform $a / b=0.9$ (open triangles). For the linear gradient in $a / b, W$ is defined as the length of the velocity-weakening region, $h^{*}$ and $T$ are defined using the average value of $a / b$ within that region (0.9), and the average slip speed at its midpoint is $v^{\infty} / 3$ (from equation (35) below).

[52] The range of normalized periods in Figure 13c exceeds a factor of 10 at $W / h_{d r}^{*}=6$ and a factor of 40 at $W / h_{d r}^{*}=12$. However, after normalizing $W$ by $h^{*}$ and the period by $T$, this range is reduced to a factor of 2 or less (Figure 13d), and furthermore coincides with (but extends to much larger $\left.W / h^{*}\right)$ the no-dilatancy trend of Rubin [2008]. While the increase in normalized period with $W / h^{*}$ in Figure $13 \mathrm{~d}$ is not fully understood, it appears to be related to the time it takes the slow event to traverse the (velocity weakening) fault.

[53] To summarize the membrane diffusion results, we note that in numerical simulations the simple result given by equation (30) does a remarkably good job of predicting the conditions of slow versus fast slip. Slow slip is favored by nearly velocity neutral friction parameters ( $a / b$ close to 1$)$, by strong dilatancy (large $\varepsilon$ ), and by low effective normal stress. While these qualitative results are expected to apply more generally, the stability boundary $\mathcal{E}_{\text {crit }}=1-\frac{a}{b}$ holds only for the model membrane diffusion system. We thus turn attention next to the presumably more representative system with full diffusion of pore fluid from the surroundings into the fault zone.

\section{Homogeneous Diffusion Calculations}

[54] In this section we extend the isothermal membrane diffusion results to consider homogeneous pore pressure diffusion into the rock adjacent to the shear zone. For the same reasons discussed in section 2.2 we neglect thermal pressurization, which is unlikely to be significant at the average slip speeds active in slow slip events. Furthermore, for simplicity we consider the limit of a vanishingly thin shear layer, $h \rightarrow 0$. The governing equations are thus: the stress equilibrium equations on the fault (16), constitutive equations for dilatancy (5), the slip law form of the state evolution (3), and the isothermal form of the diffusion equations for the infinitesimal fault zone; that is equation (11) with $\Lambda=0$. The fluid flux (Neumann) boundary condition is driven by dilatancy on the fault, as in equation (11). The pore pressure on the fault $p(y=0, t)$ drives changes in effective normal stress and hence shear strength, and thus couples to the friction and elasticity equations (16). Ignoring radiation damping effects, the solutions with spatially uniform properties are dependent on the nondimensional parameters: $a / b$, $W / h_{d r}^{*}, f_{0} / b$, and $E_{p}$ (given by equation (13)). In all calculations here we take $f_{0}=0.6$, and $b=0.0167$, such that $f_{0} / b=$ 36 , and focus on how the predicted behavior varies with $a / b$, $W / h_{d r}^{*}$, and $E_{p}$.
[55] A plausible range for $E_{p}$ is obtained by considering, from lab measurements $\epsilon$ in the range of $10^{-5}$ to $10^{-4}, d_{c}$ in the range $10^{-5}$ to $10^{-4} \mathrm{~m}, \beta$ in the range 5 to $10 \times 10^{-11} 1 / \mathrm{Pa}$, and $v^{\infty} \sim 10^{-9} \mathrm{~m} / \mathrm{s}$. The thickness of the actively shearing layer at depth in subduction zones is, of course, unknown. Based on mature crustal faults we take $h$ to be in the range of $10^{-4}$ to $10^{-3} \mathrm{~m}$, such that the ratio $h / d_{c}$ is of order 10 . This gives $\epsilon h$ in the range of $10^{-9}$ to $10^{-7} \mathrm{~m}$. Hydraulic diffusivities adjacent to the plate interface are similarly poorly constrained. Measurements from strike-slip fault zones at effective stresses appropriate for the depth of shallow crustal earthquakes are within an order of magnitude of $10^{-6} \mathrm{~m}^{2} / \mathrm{s}$ [Lockner et al., 2000; Wibberly and Shimamoto, 2003]. Inferences of high fluid pressure in regions where slow slip occurs similarly imply low pore fluid transmisivities [e.g., Audet et al., 2009]. For the sake of discussion we take a range of $10^{-7}$ to $10^{-5} \mathrm{~m}^{2} / \mathrm{s}$. This yields a range of $E_{p}$ from $10^{-5} / \bar{\sigma}(\mathrm{MPa})$ to $10^{-2} / \bar{\sigma}(\mathrm{MPa})$, where $\bar{\sigma}$ is the effective normal stress. For effective normal stresses of order $500 \mathrm{MPa}$, corresponding to depths near $30 \mathrm{~km}$ and hydrostatic pore pressure, $E_{p}$ is in the range $10^{-8}$ to $10^{-5}$. However, for low effective stresses, as inferred for some slow slip areas, $E_{p}$ could be considerably larger; $10^{-6}$ to $10^{-3}$ for $\bar{\sigma}$ of $10 \mathrm{MPa}$, and as much as $10^{-2}$ for $\bar{\sigma}$ of $1 \mathrm{MPa}$.

[56] The pore pressure diffusion equation is computed with a finite difference scheme, which is coupled to the friction elasticity equations through the pore pressure (and its time derivative) on the fault. There are a number of challenges in the finite difference computation of the pore pressure. First, we require a sufficiently fine grid near the fault to resolve steep gradients in $p$ induced by dilatancy as the rupture tip passes. At the same time, we require the pore pressure to remain unchanged at $p^{\infty}$ some significant distance from the fault. This problem is rectified by choosing a logarithmic finite difference grid, such that the grid spacing is small near the fault, where gradients may be steep, but becomes more widely spaced far from the fault. Details are given in Appendix B.

[57] Figure 14 shows the normalized pore pressure as a function of fault perpendicular distance at different snapshots in time for a representative calculation. The plot is zoomed in near the fault to show the changes in this region. The pore pressure decreases as the slip front passes, and then slowly recovers as fluid flows in from the surrounding rock. Note that the logarithmic spacing in the finite difference mesh points guarantees that the steep gradients near the fault are accurately represented while at the same time the mesh extends sufficiently far from the fault (not shown) to properly represent the remote boundary condition.

[58] In order for the results to be insensitive to arbitrary initial conditions it is necessary to run simulations over many slow slip cycles. This requires the numerical procedure to be quite efficient. To accurately resolve the slip front requires the along-fault grid spacing of the order of $L_{d} / 20$; for large values of $W / h_{d r}^{*}$ this can require thousands of points in the along-fault dimension. With the logarithmic finite difference grid we have found acceptable results with of the order of 40 points in the fault-normal dimension. Systems of this size require efficient time stepping algorithms. In an explicit finite difference scheme the time steps must be less than the Courant-Friedrichs-Lewy (CFL) condition (for the diffusion 


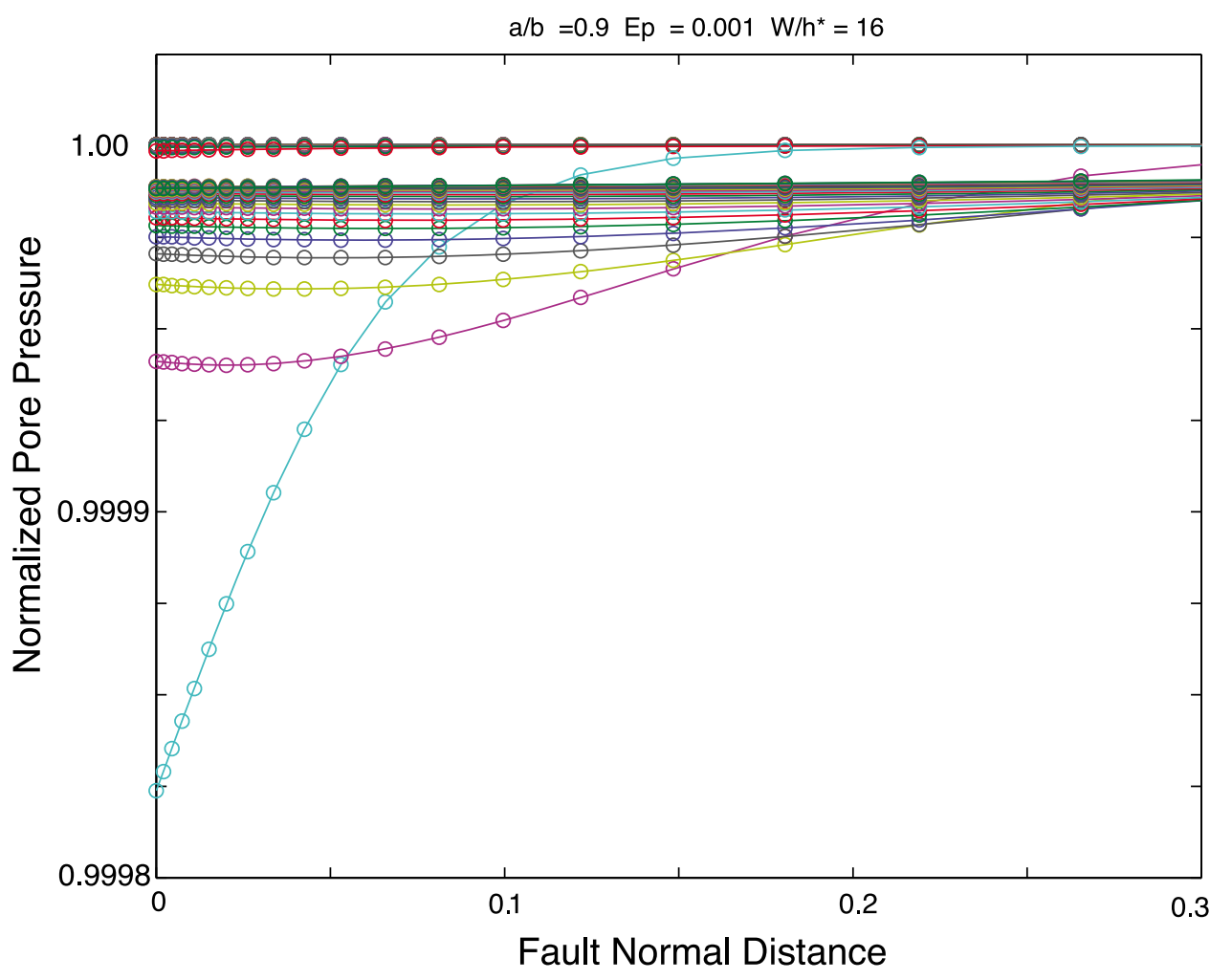

Figure 14. Numerical results illustrating pore pressure as a function of distance perpendicular to the fault at different snapshots in time. Pore pressure is normalized by the remote pore pressure, $p(y=0) / p^{\infty}$. Circles denote the finite difference grid points, more closely spaced near the fault. The finite difference grid extends to a distance of $15.8 \mathrm{~m}$ from the fault; only the first $0.3 \mathrm{~m}$ are shown here. The particular simulation is shown for $E_{p}=1.0 \times 10^{-3}, a / b=0.9$, and $W / h_{d r}^{*}=16$.

equation $\Delta t<(\Delta y)^{2} / 2 c_{\text {hyd }}$, where $\Delta y$ is the finite difference spacing). With a very fine grid near the fault the time steps in an explicit scheme become unacceptably small. A fully implicit scheme can take far bigger time steps, but requires the solution of a very large system of equations at every time step. Consequently, we developed a semi-implicit scheme that employs an explicit scheme for the frictionelasticity equations (which are not subject to the stringent stability constraints), but implicit time stepping for each one-dimensional diffusion profile normal to the fault. This involves the solution of (many) small linear systems of equations, and is consequently very efficient. This scheme permits integration of the equations over numerous slow slip cycles. Details are given in Appendix B.

[59] A sample simulation is shown for $E_{p}=1.0 \times 10^{-3}$, $a / b=0.9$, and $W / h_{d r}^{*}=16$ in Figure 15. Loading is constant slip rate at $v^{\infty}$ on the right-hand boundary ("downdip") and $10^{-3} v^{\infty}$ on the left-hand ("updip") boundary. For these parameters, slip occurs in two phases; the slow phase involves propagation away from the downdip boundary with maximum slip rates less than an order of magnitude above the plate velocity (Figure 15, blue curves). Following the end of the slow phase, a faster slip phase initiates near the middle of the velocity-weakening region, propagating first updip and subsequently bilaterally (Figure 15, red curves). Maximum slip rates during the rapid phase are less than $10^{-6} \mathrm{~m} / \mathrm{s}$, and are thus well within the quasi-static regime. A sharp decrease in fault zone pore pressure propagates with the rupture tip, and is more pronounced during the fast phase. In many respects the behavior is similar to membrane diffusion (compare to Figure 4); the main difference being that the pore pressure recovers more slowly, such that $p(y=0)$ is significantly less than the ambient pore pressure over a much longer portion of the fault than with membrane diffusion.

[60] Slip speed as a function of space and time is shown in Figure 16. Figure 16 (left) shows the slow phase propagating updip over $\sim 0.75$ years, followed by the onset of the faster transient. Figure 16 (right) zooms in on the faster phase, where slip speeds locally exceed $10^{-8} \mathrm{~m} / \mathrm{s}$. The upward phase propagates at something close to $2 \mathrm{~km} / \mathrm{d}$ for several days, while the downward phase initially lacks a clear front, but ultimately propagates at close to $9 \mathrm{~km} / \mathrm{d}$.

[61] Figure 17 illustrates slip during a single slow slip cycle. The slow phase occurs with slip migrating updip for roughly 1 year. During this time more than $3 \mathrm{~cm}$ of slip accumulates at the downdip end of the velocity weakening region, suggesting that the propagation is driven by the plate motion rather than by relaxing stored strain energy. This is confirmed by examination of the stress acting on the fault, as discussed below. The faster phase is seen to nucleate near the center of the velocity-weakening region $(x \sim 0)$ and propagate bilaterally, as seen in Figure 16.

[62] Stress accumulates during the slow phase; that is, it occurs with negative stress drop. Slip is driven by steadily 

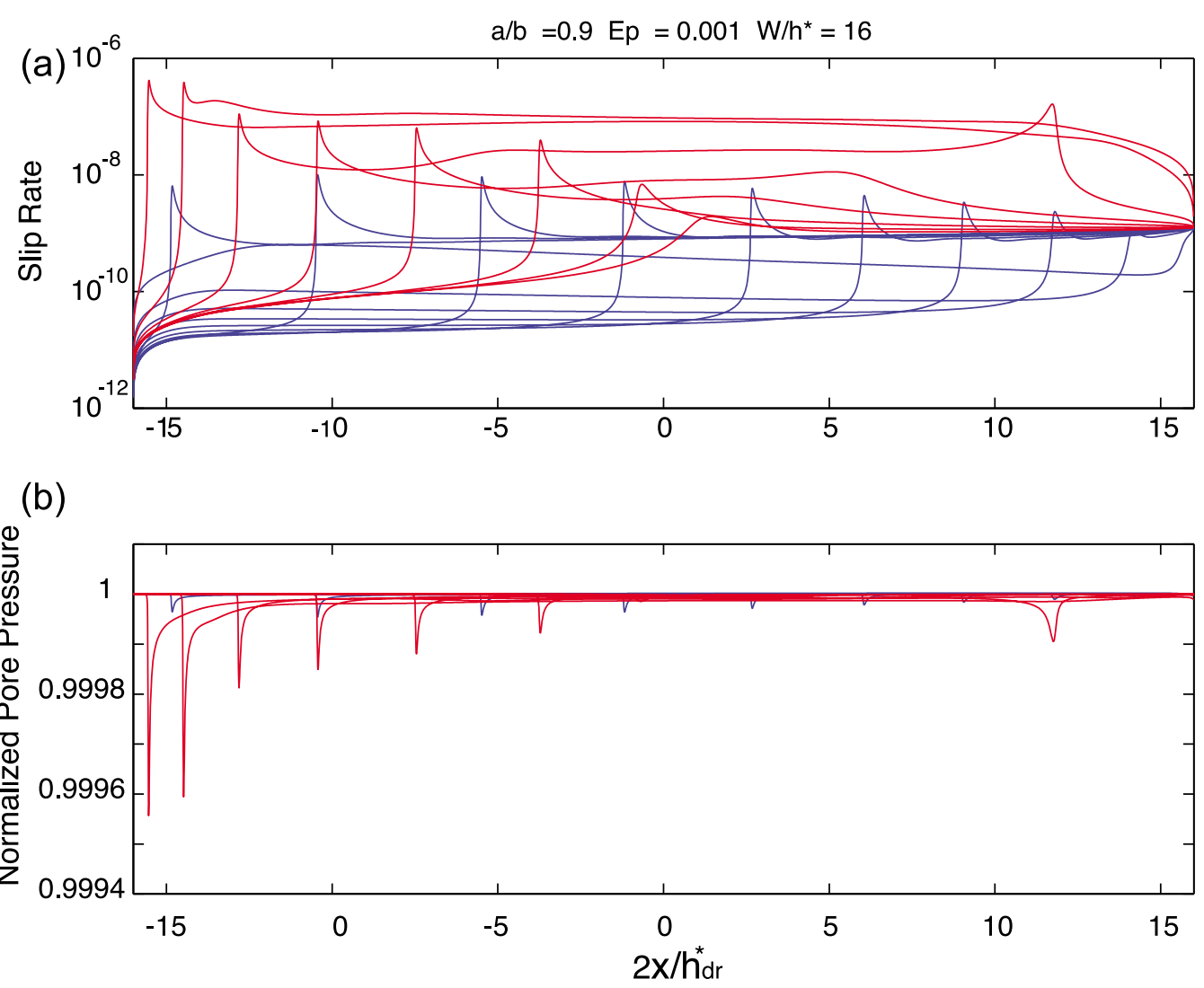

Figure 15. (a) Slip rate and (b) normalized pore pressure, $p(y=0, t) / p^{\infty}$, as a function of along-strike distance for $E_{p}=1.0 \times 10^{-3}, a / b=0.9$, and $W / h_{d r}^{*}=16$. Each curve represents a different snapshot in time, not regularly spaced. Blue curves represent slower phase, while red curves represent faster phase.

accumulating displacement at the fixed velocity boundary, rather than by relaxation of stored elastic strain. This can be seen in Figure 18. The dashed curve labeled 1 shows the stress prior to the onset of the slower phase, while curves 2 and 3 are during this phase. Notice that the stress well behind the propagating tip (the strong stress concentration) is higher at the end of the slow phase than it was prior to its onset. At the end of the slow phase the stress is uniformly high (curve 4 in Figure 18). The slow phase does, however, set the stage for the fast (but quasi-static) phase which drops the accumulated stress. At the start of the slow phase most of the fault is well below steady state $\left(v \theta / d_{c}<1\right)$; however, by the end of the slow phase much of the fault is at or near steady state (Figure 18b, curve 4). Following the cessation of fast slip (curve 5), the stress has dropped over the entire fault and the friction is well below steady state.

[63] One of the striking results of these simulations, which seems to apply for at least a modestly broad range of parameter space, is that the fault is never "locked," or slipping at many orders of magnitude below the plate velocity. Rather, there is generally some form of propagating slip, although often at rates much lower than geodetically observable slow slip events. If we associate the fast quasistatic phase with a geodetically observable transient, it is tempting to speculate that the slower phases are associated with the occurrence of nonvolcanic tremor which has been observed between major ETS events [e.g., Wech et al., 2009]. This assumes that tremor is associated with locally acceler- ated slip, which may or may not be detected geodetically. At present there is no accepted physical model for tremor itself.

[64] It is also worth noting for this particular simulation that at the midpoint of the velocity weakening region $\left(x / h^{*}=0\right)$, roughly half of the slip $(\sim 1 \mathrm{~cm})$ accumulates during the fast phase, whereas the other half accumulates in between fast events, during the slow phase (Figure 17). All together the accumulated slip (during both phases of this slow slip cycle) amount to only $2 / 3$ of the relative plate motion $\left(v^{\infty}\right)$ during this time interval. That is, only roughly $1 / 3$ of the plate motion is accommodated by the fast slip phase (at the midpoint of the slipping region). Any model in which slow slip cycles beneath a locked zone are driven by stress accumulating due to deeper plate motion must accumulate a slip deficit relative to the plate velocity, as illustrated for example in Figure 17.

[65] Slip as a function of time is illustrated in Figure 19, averaged over either the full velocity weakening region, or only the updip half of the velocity weakening region. Figure 19 emphasizes that only a fraction of the total moment that accumulates during a model slow slip cycle occurs during the rapid phase that is most clearly associated with a slow slip event. For this simulation there are four events in 4 years, for a recurrence interval of roughly 1 year. The average slip accumulated during the 4 year interval $(\sim 8 \mathrm{~cm})$ is roughly half of the net plate motion during this period $(4 \mathrm{~cm} / \mathrm{yr} \times$ 4 years $=16 \mathrm{~cm}$ ). If one considers only the updip half of the velocity weakening region, where the slip events appear 


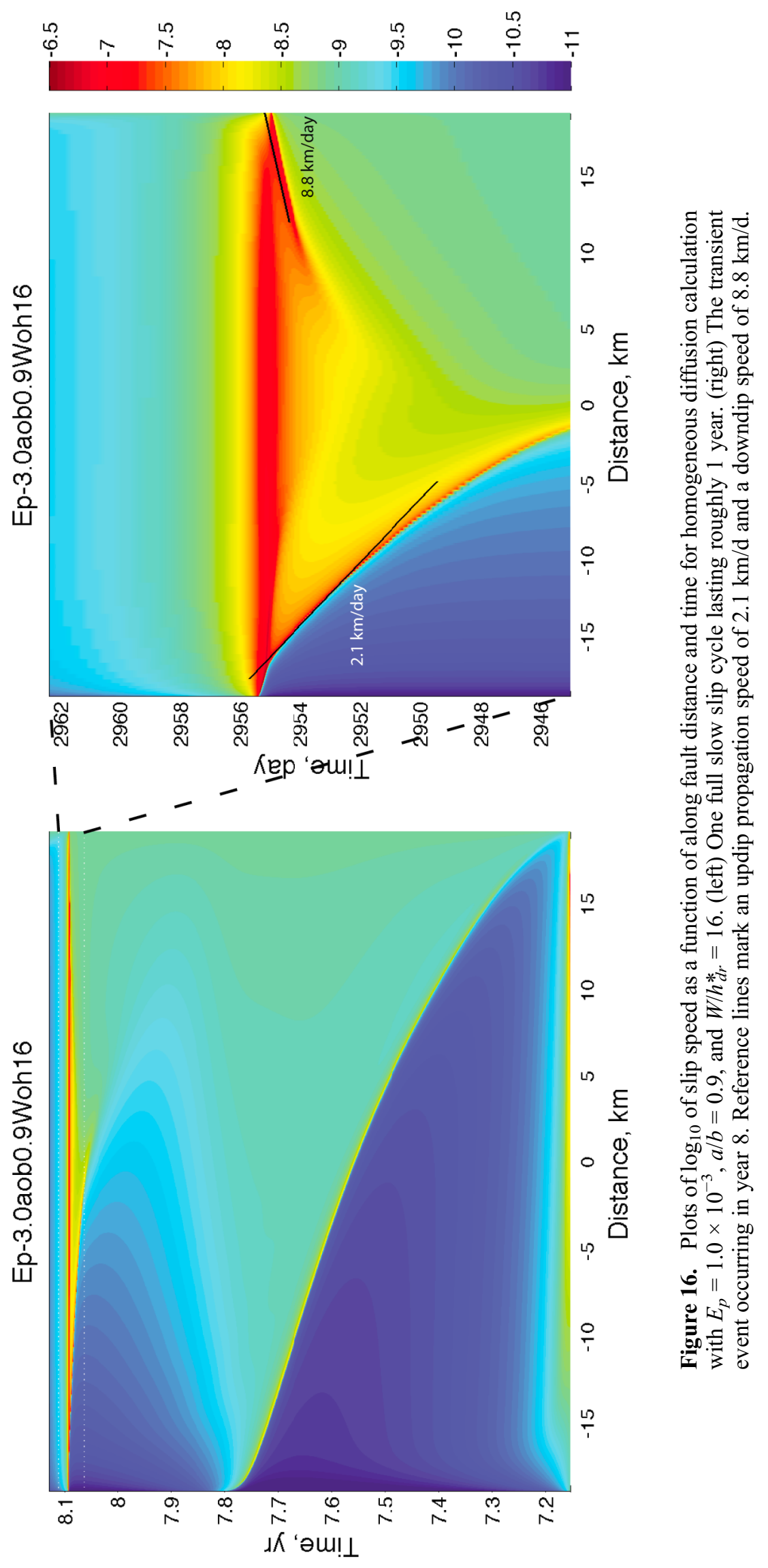




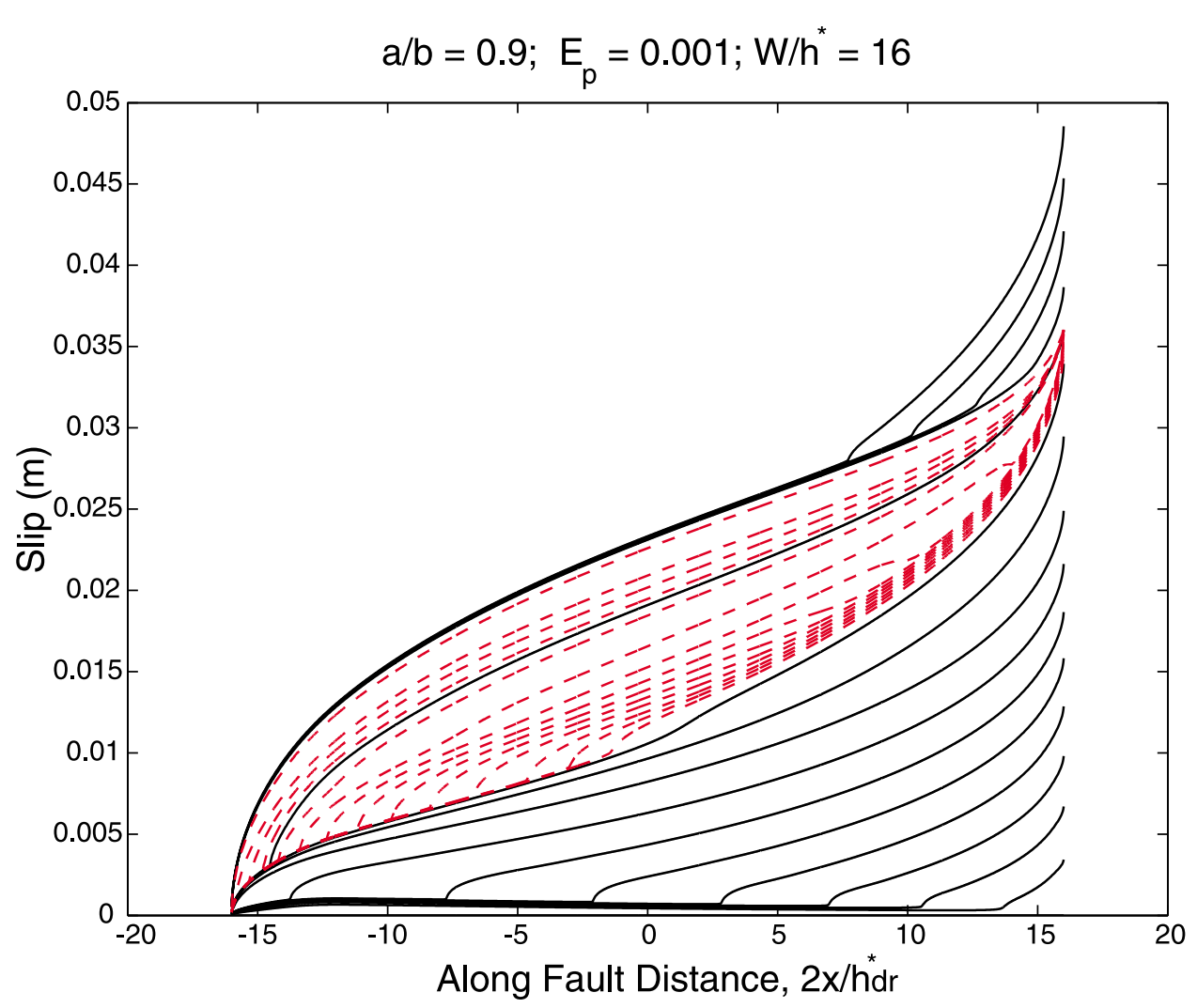

Figure 17. Slip as a function of along-strike distance for $E_{p}=1.0 \times 10^{-3}, a / b=0.9$, and $W / h_{d r}^{*}=16$. Curves are not at regularly spaced time intervals; however, the black curves are separated by roughly 0.1 years. The red dashed curves correspond to times when the average slip speed exceeds $10^{-9} \mathrm{~m} / \mathrm{s}$, roughly the plate velocity, and are mostly at intervals of roughly 2 days, although in some cases they are as short as $2 \mathrm{~h}$ apart.

more impulsive, then the accumulated slip is only roughly one third of the net relative plate motion.

[66] In order to determine how observable quantities vary as a function of the governing nondimensional parameters, we examined how the normalized moment rate and the period of the moment oscillations vary as a function of $W / h_{d r}^{*}$ for fixed $a / b=0.9$ and $E_{p}=1 \times 10^{-3}$. We define a normalized moment rate as the moment rate over the full velocity weakening region, divided by the moment rate corresponding to slip at constant shear stress over that region. For constant shear stress acting on a two-dimensional fault of width $W$ in a full space, subject to boundary conditions $v(x=W / 2)=v^{\infty}$ and $v(x=-W / 2)=0$, the slip rate distribution, is

$$
v(x)=\left(v^{\infty} / \pi\right)\left[\sin ^{-1}(2 x / W)+\pi / 2\right]
$$

[e.g., Segall, 2010, equation 12.3]. The steady state moment rate is thus $\dot{M}_{s s}=v^{\infty} W / 2$. The normalized moment rate for $a / b=0.9$ and $E_{p}=1 \times 10^{-3}$ is shown in Figure 20a. For $W /$ $h_{d r}^{*}=6$ the behavior is purely periodic with a period of $\sim 0.25$ years. For $W / h_{d r}^{*}=4$ the period is the same; however, the oscillations slowly decay. For larger $W / h_{d r}^{*}$ the $20 \%$ to $80 \%$ range of $\dot{M}_{\max } / \dot{M}_{s s}$ is shown with vertical bars. There is considerable subjectivity in plotting these data. We first eliminate early cycles to reduce dependence on initial con- ditions. In some cases it is clear when a stable limit cycle is reached, in others it is not at all clear. Secondly, we eliminate subsidiary moment rate peaks that are very close in time to neighboring larger peaks. Finally, we threshold such that normalized moment rate excursions below some cutoff are not recorded, as these are unlikely to be observed geodetically. The effect of thresholding can be to introduce a period doubling with increasing $W / h_{d r}^{*}$. This is well illustrated, for example, at $W / h_{d r}^{*}=10$ in Figure 20b. With a threshold of $2 \dot{M}_{s s}$, the smaller excursions are excluded, and the cycles are extremely periodic with $T=0.72$ years. The moment rate shows little variability with $\dot{M}_{\text {max }} / \dot{M}_{s s} \approx 4.3$. However, with a threshold of $\dot{M}_{s s}$ the smaller moment rate excursions are included and the period drops to $T=0.32$ to 0.4 years, and the moment rate spans the indicated range. With increasing $W / h_{d r}^{*} \gtrsim 15$ the longer-period oscillations dominate the moment rate function.

[67] For comparison Rubin [2008, Figure 9] finds that without dilatancy, and $a / b=0.9, \dot{M}_{\max } / \dot{M}_{s s}$ is roughly 6 for $W / h_{d r}^{*}=4$, but that the simulations become inertially limited at $W / h_{d r}^{*}$ of slightly less than 5. (These calculations include a transitional region of velocity strengthening friction between the velocity-weakening region and the imposed constant velocity boundary condition. However, Rubin [2008] shows that the presence of the velocity strengthening region does not qualitatively alter the behavior.) In contrast, with dilatancy, and $E_{p}=1 \times 10^{-3}$, the behavior remains stable at least 


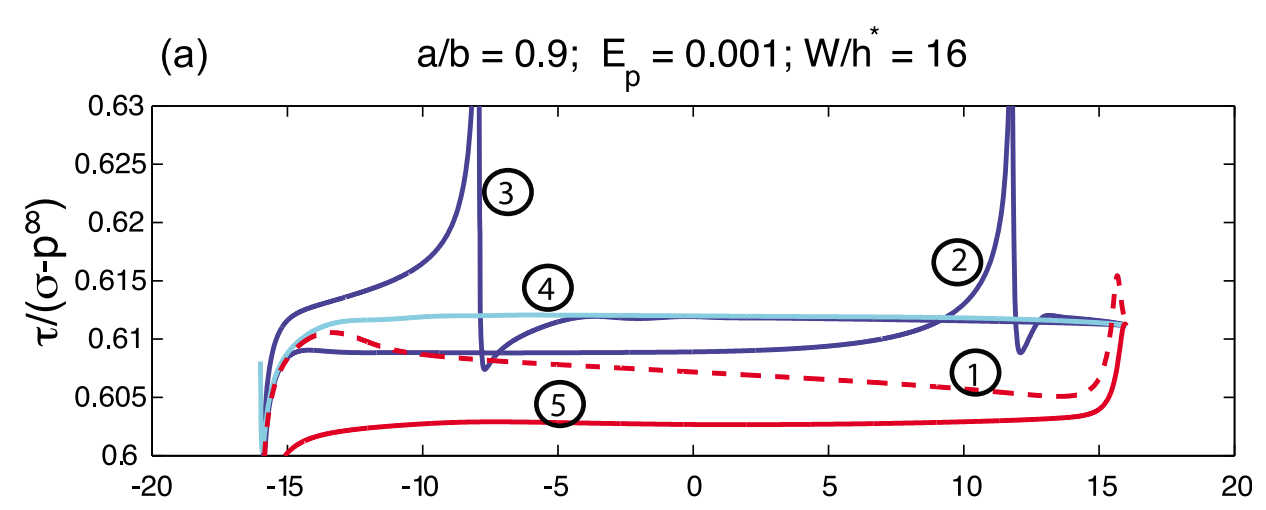

(b)

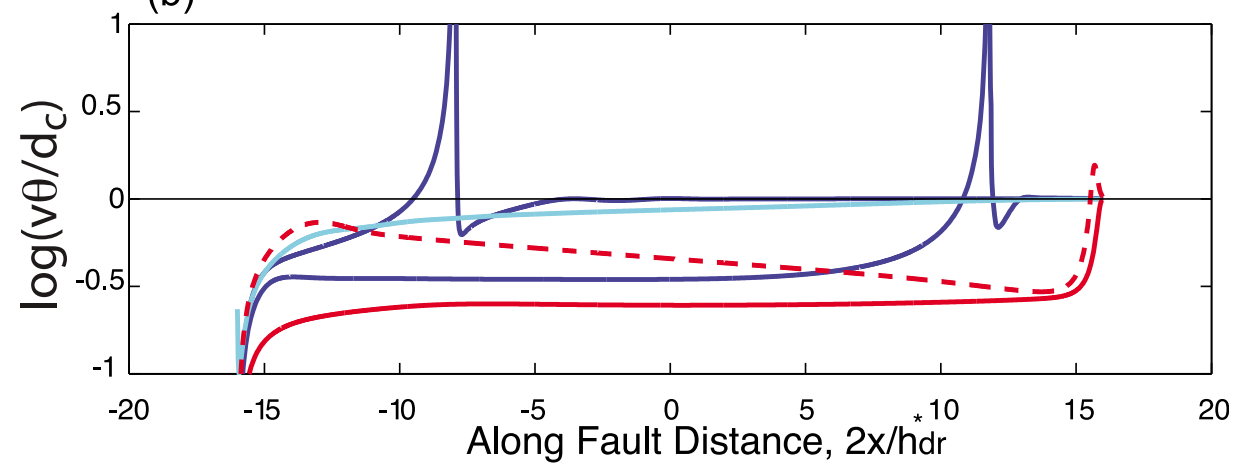

Figure 18. (a) Stress as a function of along fault position for the simulation illustrated in Figure 15. Curve 1 (dashed red) is before the start of the "slow phase." Curves 2 and 3 (solid blue) are during the slow phase; the strong stress concentration marks the tip of the slipping zone. Curves 4 (cyan) and 5 (solid red) are at the ends of the slow and fast phases, respectively. (b) Plot of $\ln \left(v \theta / d_{c}\right)$ as a function of along fault position. Colors correspond to Figure 18a.

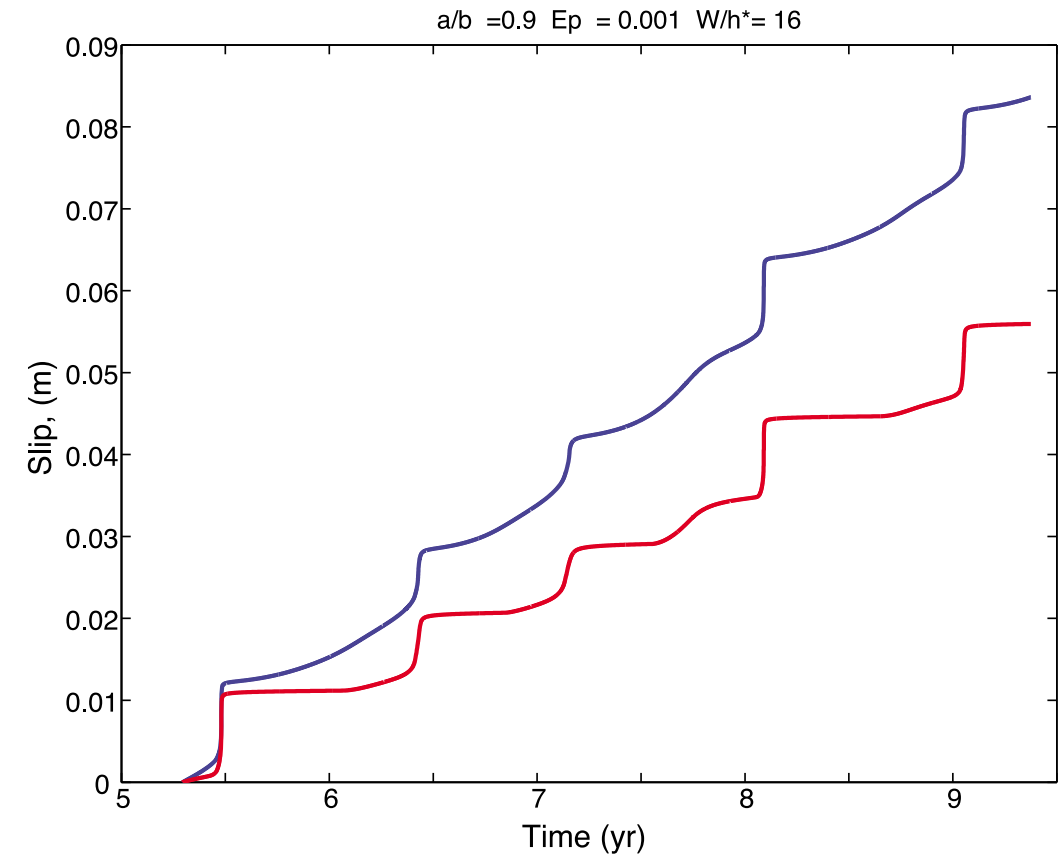

Figure 19. Average slip as a function of time for slow slip simulation with $E_{p}=1.0 \times 10^{-3}, a / b=0.9$, and $W / h_{d r}^{*}=16$. Blue curve illustrates the slip averaged over the full velocity weakening region. Red curve illustrates the slip averaged over the "updip" half of the velocity weakening region. 


$$
a / b=0.9 ; E_{p}=0.001
$$

(a)

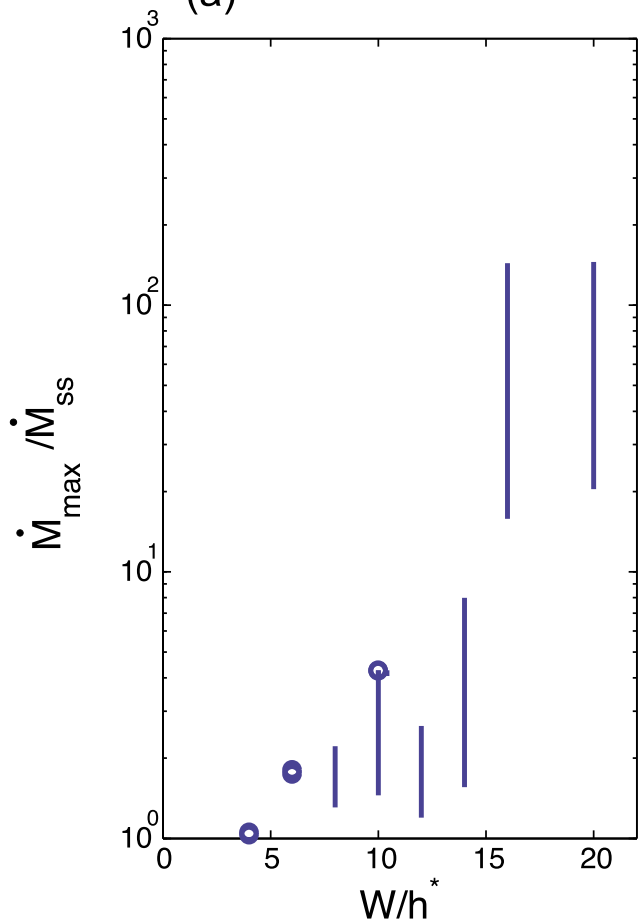

(b)

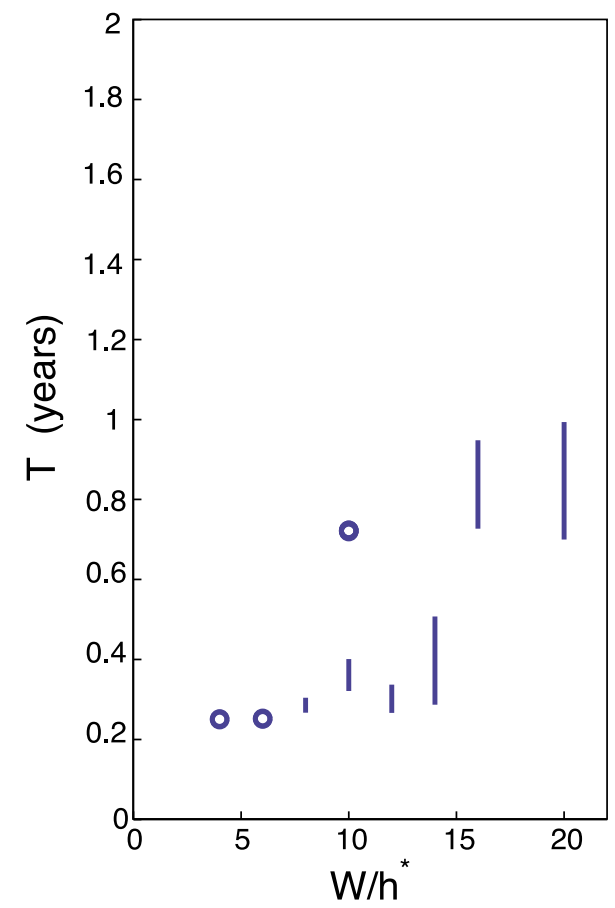

Figure 20. Normalized (a) moment rate $\dot{M}_{\max } \dot{M}_{s S}$ and (b) period as a function of normalized fault width $W / h_{d r}^{*}$ for fixed values of $a / b=0.9$ and $E_{p}=1 \times 10^{-3}$. For periodic solutions the data are shown with circles. For quasiperiodic behavior the $20 \%$ to $80 \%$ range is shown. The solution at $W / h_{d r}^{*}=4$ exhibits decaying oscillations. Two solutions are shown for $W / h_{d r}^{*}=10$, to illustrate period doubling; the very periodic result corresponds to a higher moment rate threshold.

for $W / h_{d r}^{*}$ less than 20 (Figure 20a). Indeed, a simulation with $a / b=0.9, E_{p}=3.16 \times 10^{-3}$, and $W / h_{d r}^{*}=50$ exhibited stable slip, with maximum slip speed on the order of $2.3 \times$ $10^{-7} \mathrm{~m} / \mathrm{s}$, thus demonstrating that very long slip zones can be stabilized by dilatancy. For $a / b=0.9$, and $E_{p}=3 \times 10^{-3}$, $\dot{M}_{\max } / \dot{M}_{s s}$ falls within the geodetically observed range of 10 to 100 for $W / h_{d r}^{*}$ in the range of 10 to at least 20 . We expect that for fixed $E_{p}$, and $W / h_{d r}^{*}$, the normalized moment rate will increase (decrease) for lower (higher) ratios of $a / b$, as shown in Rubin [2008] neglecting dilatancy.

[68] The dependence of the normalized moment rate and the period between slow slip events is shown as a function of $E_{p}$ in Figure 21 for fixed $a / b=0.9$ and $W / h_{d r}^{*}=12$. Note that without dilatancy this is roughly twice the maximum stable fault width according to Rubin [2008, Figure 9]. Relative to the result for $E_{p}=10^{-3}$, decreasing $E_{p}$ increases the normalized moment rate and the period. The latter arises due to the larger stress drops that occur during the "fast" phase when $E_{p}$ is small. For $E_{p}=10^{-4}, \dot{M}_{\max } / \dot{M}_{s s} \sim 0.3$ to $1.0 \times 10^{4}$; that is, average slip rates are a factor of $10^{4}$ above the plate velocity, and the period increases to roughly 1.4 years. Increasing $E_{p}$ decreases the normalized moment rate, such that for $E_{p}=6 \times 10^{-4}$, the moment rate is a factor of 100 over the steady state rate, and the period is slightly less than 1 year. Increasing $E_{p}$ to $10^{-3}$ drops the normalized moment rate close to unity and decreases the period by roughly a factor of 2 , reflecting the period doubling phe- nomenon discussed above. Note that decreasing $E_{p}$ could result from either decreasing $\epsilon h$, increasing the effective normal stress, compressibility, hydraulic diffusivity, or $d_{c}$.

[69] These results demonstrate that, as expected, inclusion of a more accurate diffusion model does not fundamentally change our conclusion that modest dilatancy can stabilize slip against inertial instability.

\section{Relationship Between Membrane and Homogeneous Diffusion}

[70] We have analyzed two models for the effects of dilatancy on fault stability that differ solely in the manner in which pore fluid transport is approximated. The behavior is qualitatively similar, but different in detail as membrane diffusion depends on the two dimensionless parameters $\mathcal{E}$ and $\mathcal{U}$, while homogeneous diffusion depends on $E_{p}$. It is desirable to understand more quantitatively how these models relate to one another. We first explore this issue by considering behavior in both models near neutral stability. Next we contrast the pore pressure response to abrupt changes in slip speed in these two models.

[71] From the definitions of $E_{p}$ and $\mathcal{E}$

$$
E_{p}=\frac{b}{\sqrt{2} f_{0}} \mathcal{E} \sqrt{\frac{h^{2} v^{\infty}}{c_{h y d} d_{c}}}
$$




$$
\mathrm{a} / \mathrm{b}=0.9 ; \mathrm{W} / \mathrm{h}^{*}=12
$$
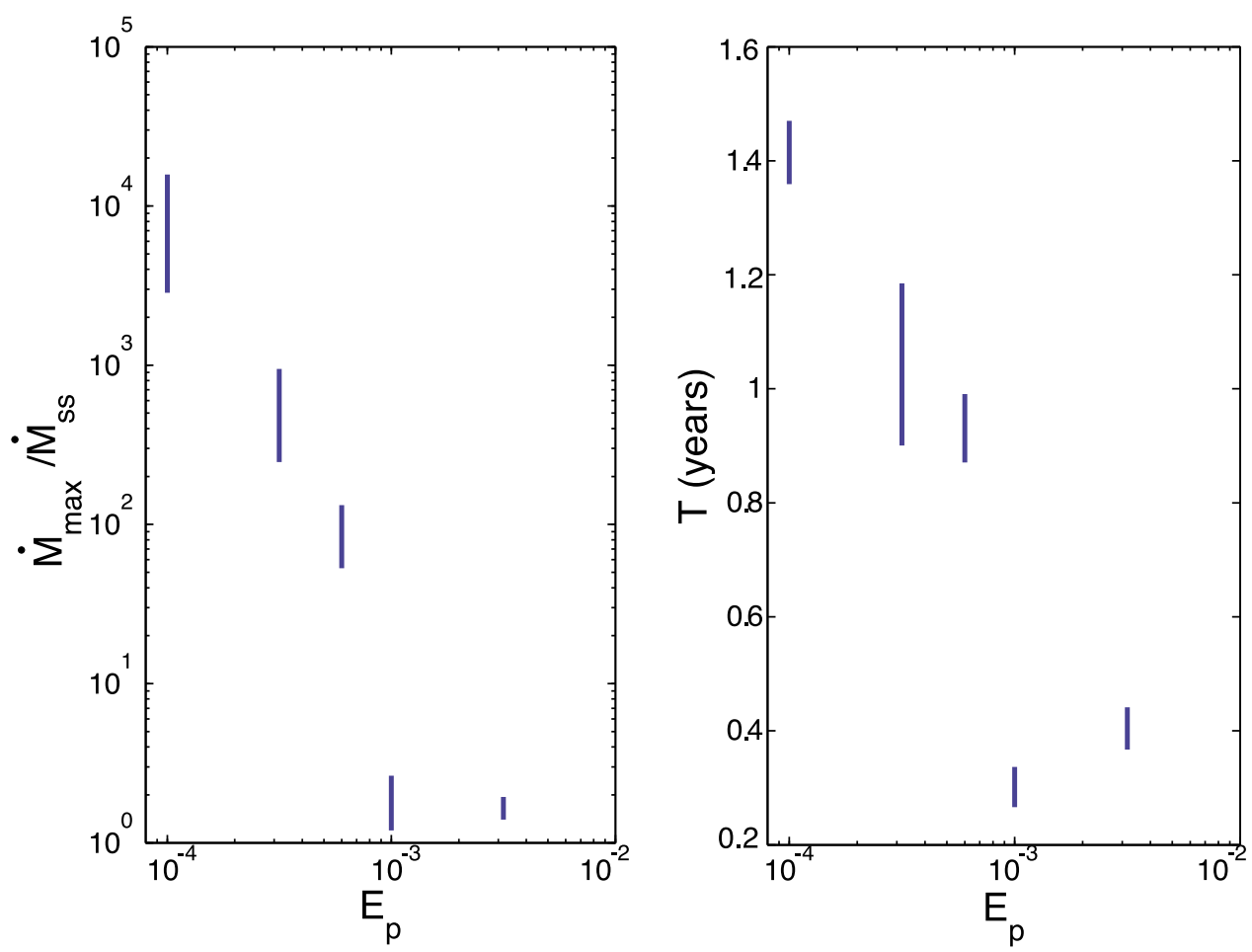

Figure 21. Normalized moment rate $\dot{M}_{\text {max }} / \dot{M}_{s s}$ and period between slow events, as a function of $E_{p}$, for fixed $W / h_{d r}^{*}=12$, and $a / b=0.9$.

Noting that $t_{f}=h h_{w} / 2 c_{h y d}$, from (15) we write

$$
E_{p}=\frac{b}{\sqrt{2} f_{0}} \mathcal{E} \mathcal{U}^{1 / 2}\left(\frac{h}{h_{w}}\right)^{1 / 2}
$$

In the membrane diffusion model the diffusion distance is always the wall thickness $h_{w}$. In the homogeneous diffusion model at neutral stability, it is the characteristic diffusion distance during a half-period of oscillation. Comparing the two models at neutral stability suggests equating these two distances, that is

$$
h_{w}=\varpi \sqrt{c_{h y d} T_{h d}\left(E_{p}\right)}
$$

where the period at neutral stability $T_{h d}$ is a function of $E_{p}$, and $\varpi$ is a constant of order unity that accounts for the scaling in the characteristic diffusion length and the fact that fluid flow is unidirectional during a half, rather than full, cycle. Rearranging (36) for $h$ in terms of $E_{p}$ and $\mathcal{E}$, and substituting this result, along with (38), into (37) yields

$$
E_{p}=\frac{b}{f_{0} \varpi} \mathcal{E} \mathcal{U}\left[\tilde{T}_{h d}\left(E_{p}\right)\right]^{-1 / 2}
$$

where $\tilde{T}$ is the nondimensional period $T v^{\infty} / d_{c}$. Equation (39) provides the first relationship between $E_{p}$ and the membrane diffusion parameters $\mathcal{E}$ and $\mathcal{U}$.

\subsection{Large $E_{p}$ Limit}

[72] In the limit $\mathcal{E}>\mathcal{E}_{\text {crit }}$ and $\mathcal{U} \gg 1$ equation (31) gives $h_{m d}^{*} / h_{d r}^{*} \approx \mathcal{U}$, where $h_{m d}^{*}$ is the critical nucleation dimension for membrane diffusion. Comparing to the large $E_{p}$ limit for homogeneous diffusion, equation (C25) in Appendix C, leads to

$$
\mathcal{U} \approx\left(1-\frac{a}{b}\right)^{-2}\left(\frac{f_{0} E_{p}}{\sqrt{2} b}\right)^{2}=\frac{\tilde{T}_{h d}}{2 \pi},
$$

where the latter relation makes use of equation (C21). This shows that in dimensional terms $t_{f} \sim T_{h d} / 2 \pi$; the characteristic diffusion time in the membrane diffusion model should be chosen equal to the (circular) period at neutral stability in the homogeneous diffusion model.

[73] The nondimensional period at neutral stability for homogeneous diffusion is given by equation (C21) and for membrane diffusion by (34). Equating these and making use of (40) leads to

$$
\mathcal{E}=2\left(1-\frac{a}{b}\right)=2 \mathcal{E}_{\text {crit }} .
$$

Thus, in order to approximate homogeneous diffusion behavior near neutral stability for large $E_{p}$ with the membrane diffusion model, one chooses $\mathcal{U}$ from equation (40), and sets $\mathcal{E}=2(1-a / b)$. The former specifies that the characteristic diffusion time across the wall zone is proportional to the period at neutral stability in the homogeneous diffusion model, while the latter that $\mathcal{E}$ exceeds $\mathcal{E}_{\text {crit }}$ so that the critical 

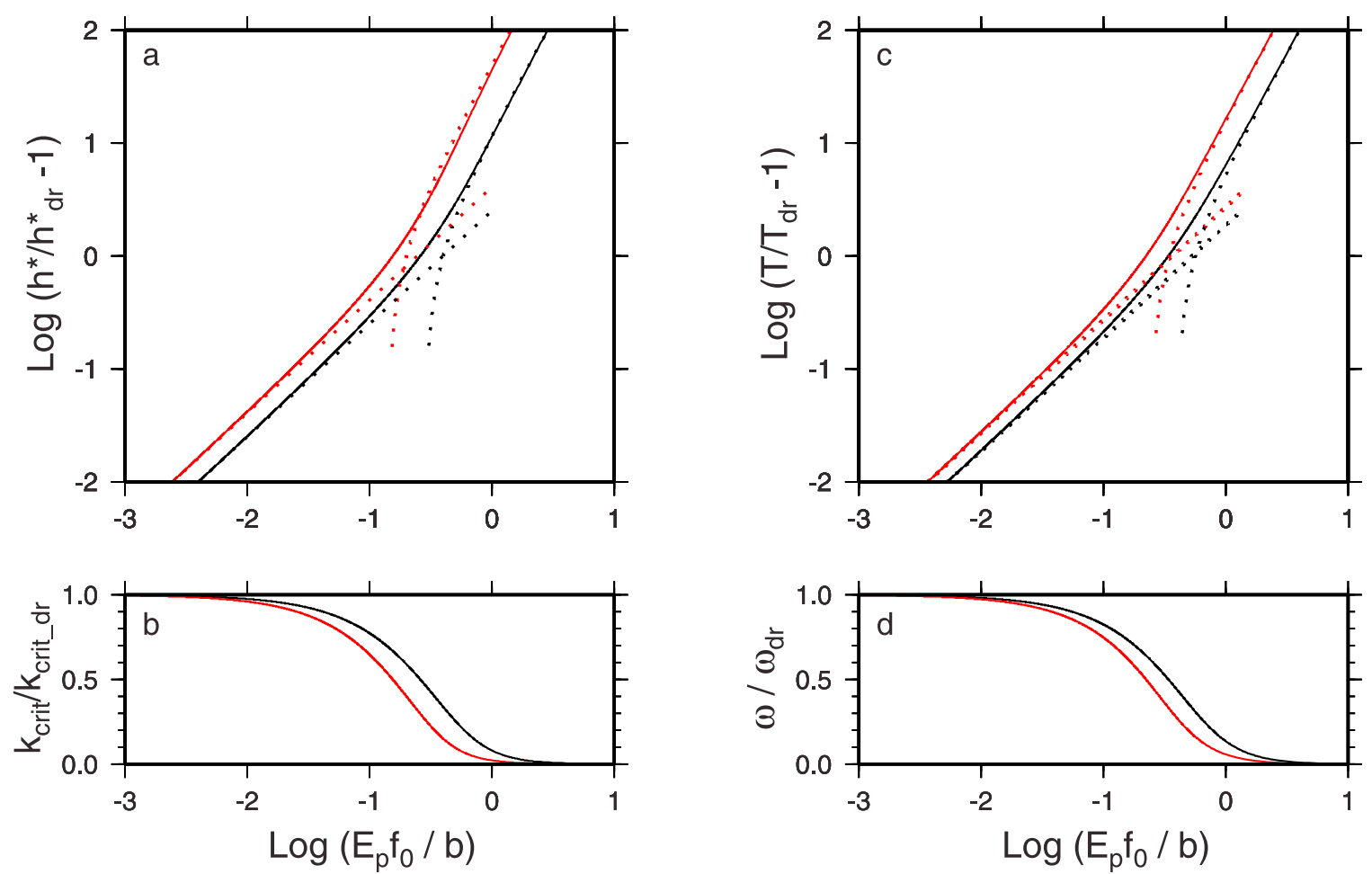

Figure 22. Plots of (a) $\ln \left(h^{*} / h_{d r}^{*}-1\right)$, (b) $k_{\text {crit }} / k_{\text {crit } d r}$, (c) $\ln \left(T / T_{d r}-1\right)$, and (d) $\omega / \omega_{d r}$, as functions of $\ln \left(E_{p} f_{0} / b\right)$, from equations $(\mathrm{C} 10)$ and $(\mathrm{C} 12)$, for $a / b=0.8$ (black lines) and $a / b=0.9$ (red). Dotted lines in Figures 22a and 22c show asymptotic limits for small $E_{p}$ (equations (C16) and (C18)) and large $E_{p}$ (equation (C21), after normalization by the drained value and equation (C25)).

nucleation length $h_{m d}^{*}$ becomes unbounded in the undrained limit, as it does in the homogeneous diffusion case (Figure 22 and equation (C25)).

[74] We also check consistency with equation (38), which states that the appropriate wall thickness, $h_{w}$, is related to the period of neutral stability in the homogeneous diffusion case, consistent with the result in equation (40). Making use of (40), equation (39) reduces to

$$
E_{p}=\frac{b}{f_{0} \varpi} \frac{\mathcal{E}}{2 \pi}\left[\tilde{T}\left(E_{p}\right)\right]^{1 / 2} .
$$

From the result for $\tilde{T}_{h d}$ in equation (C21), we find that

$$
\mathcal{E}=2 \varpi \sqrt{\pi}\left(1-\frac{a}{b}\right),
$$

which agrees with (41) for $\varpi=1 / \sqrt{\pi}$.

\subsection{Low $E_{p}$ Limit}

[75] Note that model slow slip behavior reminiscent of natural events occurs in the low- $E_{p}$ limit (see Appendix C). For example, $E_{p} \leq 10^{-3}$ implies $E_{p} f_{0} / b \lesssim 3 \times 10^{-2}$ (Appendix $\mathrm{C}$ and Figure 22). Because $E_{p}$ is defined at a slip speed of $v^{\infty}$, the fault can be essentially drained as a slow slip event nucleates, but moderately undrained for speeds 1-2 orders of magnitude larger, as discussed further in section 5.3. Equating the drained asymptotic results for $h^{*}$ in the membrane (A5) and homogeneous diffusion models (C18), as well as the periods at neutral stability, equations (A6) and (C16), yields two equations in the unknowns $\mathcal{E}$ and $\mathcal{U}$. These can be written compactly letting $\omega \equiv \sqrt{b / a-1}$, the nondimensional drained frequency at neutral stability, and $E_{p}^{\prime} \equiv f_{0} E_{p} / \sqrt{2} b$, as

$$
\begin{aligned}
\omega^{2} \mathcal{E} \mathcal{U} & =E_{p}^{\prime}\left(\omega^{3 / 2}+\omega^{1 / 2}\right) \\
\omega^{2} \mathcal{E} \mathcal{U}^{2} & =E_{p}^{\prime} \omega^{1 / 2} .
\end{aligned}
$$

Solving for $\mathcal{U}$ leads to

$$
\mathcal{U}=(\omega+1)^{-1}=\left[\sqrt{\frac{b}{a}-1}+1\right]^{-1} .
$$

Thus, in the drained limit the appropriate choice of the nondimensional characteristic diffusion time in the membrane diffusion model $\tilde{t}_{f}$ is related to the nondimensional drained period at neutral stability, $\tilde{T}_{d r}$

$$
\tilde{t}_{f}=\left[\frac{2 \pi}{\tilde{T}_{d r}}+1\right]^{-1}
$$

Substituting (45) into (44) yields the estimate of $\mathcal{E}$ in the drained limit.

$$
\mathcal{E}=\frac{f_{0} E_{p}}{\sqrt{2} b} \frac{\left(\sqrt{\frac{b}{a}-1}+1\right)^{2}}{\left(\frac{b}{a}-1\right)^{3 / 4}}
$$


Returning to equation (39), making use of (45), and taking the drained limit $\tilde{T}_{d r}$ for $\tilde{T}_{h d}\left(E_{p}\right)$ leads to

$$
\mathcal{E}=\frac{\sqrt{2 \pi} f_{0} \varpi E_{p}}{b} \frac{\left(\sqrt{\frac{b}{a}-1}+1\right)}{\left(\frac{b}{a}-1\right)^{1 / 2}} .
$$

For both equations (47) and $(48), \mathcal{E}$ is proportional to $E_{p}$, although the constants are somewhat different. In equation (47), $b \mathcal{E} / f_{0} E_{p}$ ranges from 4.5 to 6.6 for $a / b$ between 0.8 and 0.9 ; while in equation $(48), b \mathcal{E} / f_{0} E_{p}$ ranges from 4.2 to 5.6 for the same range in $a / b$ and $\varpi=\pi^{-1 / 2}$. In summary, for the low $E_{p}$ case, the appropriate characteristic diffusion time in the membrane model depends on the drained period at neutral stability, as in equation (46), while the appropriate choice of $\mathcal{E}$ is given by (47).

\subsection{Pore Pressure Change Following Velocity Steps}

[76] The previous analysis describes the relationship between the membrane diffusion and homogeneous diffusion models near neutral stability. Also of interest is the behavior far from neutral stability where propagating slip events are observed in both models. In many of the simulations these events appear as nearly step changes in slip speed (at least on a log scale). This is observed in Figures 5, 10, and 12 for membrane diffusion, and in Figure 15 for the homogeneous diffusion case, although for the latter the slip speed decays more substantially behind the slip front.

[77] In order to elucidate the difference in behavior between the two diffusion models we examine the pore pressure response to a step change in slip speed, $v(t)=v H(t)$ for both models. The derivations are given in Appendix D. For membrane diffusion we find (Appendix D, equation (D5)) that the fault zone pore pressure following the velocity step is given by

$$
\begin{aligned}
p^{m d}(t) & =-\frac{\epsilon}{\beta} \ln \left(\frac{v \theta_{i}}{d_{c}}\right) \frac{v t_{f}}{d_{c}-v t_{f}}\left(e^{-\delta / d_{c}}-e^{-t / t_{f}}\right) ; \quad \frac{v t_{f}}{d_{c}} \neq 1 \\
& =-\frac{\epsilon}{\beta} \ln \left(\frac{v \theta_{i}}{d_{c}}\right) \frac{\delta}{d_{c}} e^{-v t / d_{c}} ; \quad \frac{v t_{f}}{d_{c}}=1
\end{aligned}
$$

where $\theta_{i}$ is the state variable prior to the velocity step, and slip is $\delta=v t$. The pore pressure drop accumulates exponentially with $\delta / d_{c}$ and then decays, as pore fluid flows into the fault zone, with increasing slip as $\delta / v t_{f}$. The maximum pore pressure change is

$$
\begin{aligned}
p_{\max }^{m d} & =-\frac{\epsilon}{\beta} \ln \left(\frac{v \theta_{i}}{d_{c}}\right)\left(\frac{v t_{f}}{d_{c}}\right)^{-\frac{d_{c}}{v_{f}-d_{c}}} ; \quad \frac{v t_{f}}{d_{c}} \neq 1 \\
& =-\frac{\epsilon}{\beta} \ln \left(\frac{v \theta_{i}}{d_{c}}\right) e^{-1} ; \quad \frac{v t_{f}}{d_{c}}=1
\end{aligned}
$$

Define $\Upsilon \equiv v t_{f} / d_{c}=\left(v / v^{\infty}\right) \mathcal{U}$, and the function $\Gamma(\Upsilon)$ by $\Gamma(\Upsilon \neq 1) \equiv \Upsilon^{1 /(1-\Upsilon)} ; \Gamma(\Upsilon=1) \equiv 1 / e$. Note that in the limit that $\Upsilon \ll 1, \Gamma(\Upsilon) \rightarrow \Upsilon$; as $\Upsilon$ tends to zero, pore pressure dissipates faster than it accumulates. On the other hand, for
$\Upsilon \gg 1, \Gamma(\Upsilon) \rightarrow 1$. Thus, the limiting forms of (50), written in terms of $\mathcal{E}$ are:

$$
\begin{aligned}
\lim _{v t_{f} / d_{c} \ll 1} p_{\max }^{m d} & =-\frac{b \bar{\sigma}}{f_{0}} \mathcal{E}\left(\frac{v t_{f}}{d_{c}}\right) \ln \left(\frac{v \theta_{i}}{d_{c}}\right) \\
\lim _{v t_{f} / d_{c} \gg 1} p_{\max }^{m d} & =-\frac{b \bar{\sigma}}{f_{0}} \mathcal{E} \ln \left(\frac{v \theta_{i}}{d_{c}}\right)
\end{aligned}
$$

where, $\bar{\sigma} \equiv\left(\sigma-p^{\infty}\right)$. For homogeneous diffusion, on the other hand, the pore pressure on the fault is (Appendix D, equation (D14))

$$
p^{h d}(y=0, \delta)=-\frac{2 \bar{\sigma} E_{p}}{\sqrt{\pi}} \sqrt{\frac{v}{v^{\infty}}} \ln \left(\frac{v \theta_{i}}{d_{c}}\right) D\left(\sqrt{\frac{\delta}{d_{c}}}\right)
$$

where $D(z)$ is known as Dawson's Integral

$$
D(z)=e^{-z^{2}} \int_{0}^{z} e^{t^{2}} d t
$$

$D(z)$ exhibits a maximum of $\sim 0.541$ at $z \simeq 0.924$ and then decays slowly as pore pressure recovers (Figure 23). For large argument Dawson's Integral behaves as $D(z \gg 1) \sim 1 / 2 z$. The maximum pore pressure excursion in the homogeneous diffusion case is thus

$$
p_{\max }^{h d} \approx-\frac{E_{p}}{\sqrt{\pi}} \bar{\sigma} \sqrt{\frac{v}{v^{\infty}}} \ln \left(\frac{v \theta_{i}}{d_{c}}\right)
$$

Comparing to (50) the maximum pore pressure excursions in the two models are equal when

$$
E_{p} \approx \frac{\sqrt{\pi} b}{f_{0}} \mathcal{E}\left(\frac{v}{v^{\infty}}\right)^{-1 / 2} \Gamma\left(\mathcal{U} \frac{v}{v^{\infty}}\right)
$$

where the function $\Gamma$ is defined following equation (50). From a fracture mechanics perspective [e.g., Rubin and Ampuero, 2005] it is likely the integral of the induced pore pressure change with slip has a more significant effect on the propagation behavior than does $p_{\max }$. The effective fracture energy $G_{c}$ is defined as the integral of the shear strength as a function of displacement

$$
G_{c} \equiv \int_{0}^{\delta^{*}}\left[\tau(\delta)-\tau\left(\delta^{*}\right)\right] d \delta
$$

[e.g., Rice, 2006], where $\delta^{*}$ is the slip at which the strength degrades to roughly constant level. The contribution to the fracture energy from dilatancy (that is excluding the contribution from changes in friction) is computed by inserting $-f_{0} \Delta p$ for $\tau$ in (56). For membrane diffusion employing equation (49), this results in

$$
G_{c}^{m d}=\frac{f_{0} \epsilon}{\beta} \ln \left(\frac{v \theta_{i}}{d_{c}}\right) v t_{f}
$$




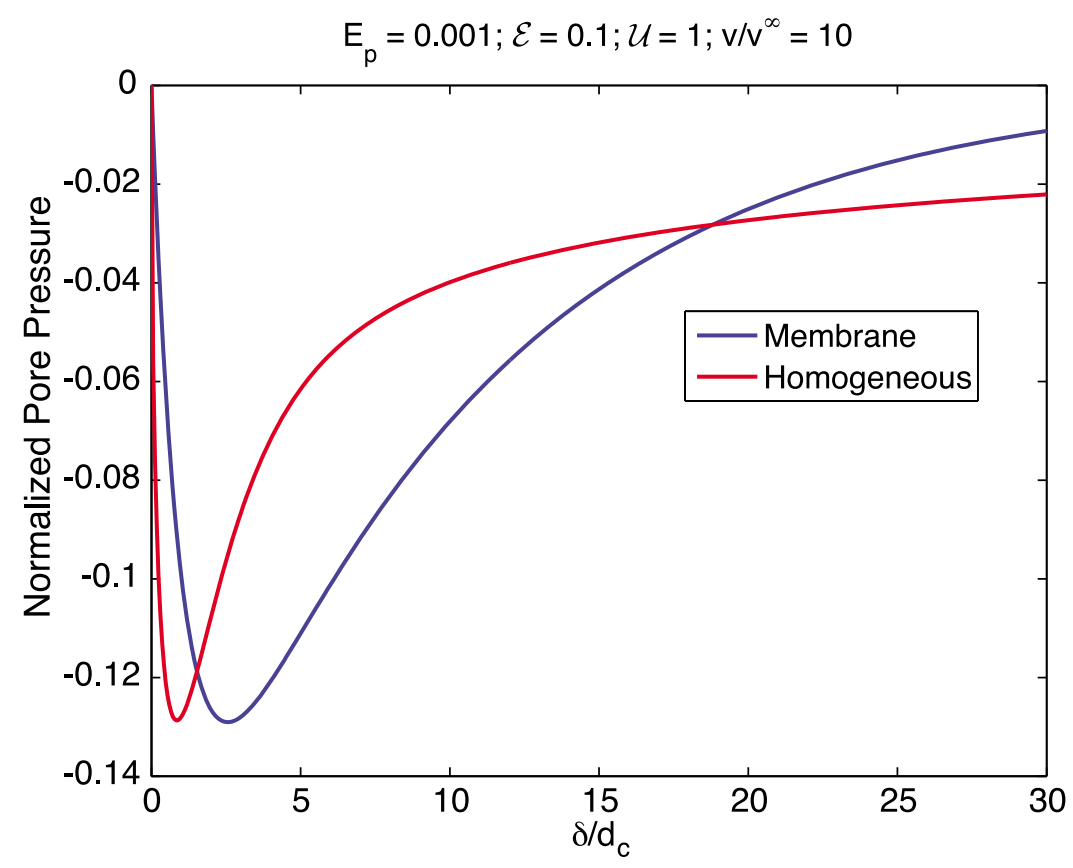

Figure 23. Comparison between pore pressure changes induced on a step increase in slip speed $v$ for membrane diffusion and homogeneous diffusion; see equations (49) and (52). In this example $E_{p}=10^{-3}$, $\mathcal{E}=0.1, \mathcal{U}=1$, and the velocity jump is a factor of 10 over the plate velocity. Also, $b=0.015$ and $f_{0}=0.6$.

which is valid for all $v t_{f} / d_{c}$. For the homogeneous diffusion case:

$$
G_{c}^{h d}=\frac{2 f_{0} \bar{\sigma} E_{p}}{\sqrt{\pi}} d_{c} \sqrt{\frac{v}{v^{\infty}}} \ln \left(\frac{v \theta_{i}}{d_{c}}\right) I\left(\delta^{*} / d_{c}\right),
$$

where

$$
I\left(\delta^{*} / d_{c}\right) \equiv \int_{0}^{\delta^{*} / d_{c}} D(\sqrt{z}) d z-\frac{\delta^{*}}{d_{c}} D\left(\sqrt{\frac{\delta^{*}}{d_{c}}}\right) .
$$

For sufficiently large argument (roughly $\delta^{*} / d_{c}>5$ ), the definite integral in (59) increases as $\sqrt{\delta^{*} / d_{c}}$. Given the asymptotic behavior of $D(z), I\left(\delta^{*} / d_{c}\right)$ scales roughly as $(1 / 2) \sqrt{\delta^{*} / d_{c}}$. For $\delta^{*} / d_{c}$ in the range of 10 to $30, I\left(\delta^{*} / d_{c}\right)$ ranges between 1.6 and 2.7. In what follows we take $\delta^{*} / d_{c}=20$, such that $I\left(\delta^{*} / d_{c}\right)=$ 2.2. We note that $I\left(\delta^{*} / d_{c}\right)$ is formally unbounded with increasing $\delta^{*}$; however, there is no paradox with regard to the numerical simulations because the slip velocity decreases substantially behind the rupture tip (Figure 15) such that the effective fracture energy is finite.

[78] We have seen in simulations (Figure 18) that prior to the faster slip phases, the slow phase brings the fault close to steady state friction at nearly the driving velocity. Thus, it is reasonable in these cases to take $\theta_{i} \approx d_{c} / v^{\infty}$. Making this assumption and normalizing the fracture energy by $\bar{\sigma} d_{c}$ we have, for membrane diffusion

$$
\frac{G_{c}^{m d}}{\bar{\sigma} d_{c}}=b \mathcal{E U}\left(\frac{v}{v^{\infty}}\right) \ln \left(\frac{v}{v^{\infty}}\right)
$$

The corresponding result for homogeneous diffusion is

$$
\frac{G_{c}^{h d}}{\bar{\sigma} d_{c}}=\frac{2 f_{0} E_{p}}{\sqrt{\pi}} I\left(\delta^{*} / d_{c}\right) \sqrt{\frac{v}{v^{\infty}}} \ln \left(\frac{v}{v^{\infty}}\right)
$$

In the membrane diffusion case the fracture energy due to dilatancy increases with the magnitude of the velocity jump as $v \ln (v)$, the $\ln (v)$ arising from the magnitude of the pore pressure change, the $v$ arising from the characteristic slip weakening scale $\left(v t_{f}\right)$, whereas in the homogeneous case the fracture energy scales with $\sqrt{v} \ln (v)$. This illustrates a fundamental limitation of the membrane diffusion model: the pore pressure recovery time $t_{f}$ is independent of the magnitude of the velocity step, whereas in homogeneous diffusion the effective recovery time scales with $d_{c} / v$, and is thus shorter for larger velocity steps. This leads to fracture energies in the membrane diffusion approximation that increase with $v$ faster than for homogeneous diffusion by a factor of $\sqrt{v}$.

[79] A complete fracture analysis, which is beyond our present scope, would include the balance between the fracture energy and the energy release rate. For drained rate-state friction behavior Rubin and Ampuero [2005] showed that for sufficiently large $v$, the energy release rate scales with $L[\ln (v)]^{2}$, where $L$ is the length of the active slip zone, while $G_{c}$ scales with $[\ln (v)]^{2}$ for the aging law, but only with $\ln (v)$ for the slip law. Thus, for the aging law only, $G_{c}$ balances the energy release rate for $L$ less than a critical length, $L_{\infty}$. If the interior of the crack drains to the background pore pressure, the Rubin-Ampuero estimate of $G$ approximately holds. In this event there is no limiting slip zone length if $G_{c}$ increases with $v$ faster than $[\ln (v)]^{2}$, which we note is 
true for both membrane and homogeneous diffusion. In detail, the slow pore pressure recovery in the homogeneous diffusion case decreases the stress drop well behind the rupture tip, thus decreasing $G$ and further stabilizing the rupture relative to the membrane diffusion case which is relatively drained in this region.

[80] Equating the apparent fracture energy in the membrane and homogeneous diffusion models leads to

$$
E_{p}=\left[\frac{\sqrt{\pi} b}{2 f_{0} I\left(\delta^{*} / d_{c}\right)}\right] \mathcal{E U}\left(\frac{v}{v^{\infty}}\right)^{1 / 2}
$$

which can be compared with equation (55). Matching both the fracture energy and the maximum pore pressure excursion requires $\Upsilon=2 I\left(\delta^{*} / d_{c}\right) \Gamma(\Upsilon)$. Since $\Gamma(\Upsilon) \leq \Upsilon$, such solutions exist only for $2 I\left(\delta^{*} / d_{c}\right)>1$. For example with, $I\left(\delta^{*} / d_{c}\right) \sim 2.2$, matching the fracture energy and peak suction occur for $\Upsilon \sim 2$, which implies $v / v^{\infty} \sim \mathcal{U}^{-1}$. While there is a rather narrow range of parameters that match both the fracture energy and peak suction, there appears to be a broader range for which they are approximately equivalent, although we have not explored this in detail.

[81] The constant term in brackets in (62) is of the order of $10^{-2}$. From this we suggest a range of $E_{p}$ that yields slip rates roughly an order of magnitude greater than the plate velocity. For example, choosing $\mathcal{E} \sim 1-a / b \sim 0.1$, and $\mathcal{U}$ on the order of 1 , leads to $E_{p}$ of roughly $2 \times 10^{-3}$, which we have already seen provides interesting behavior in the sense of producing slow slip events that are roughly an order of magnitude above plate velocity, for an appropriate range of $W / h_{d r}^{*}$. Note that $E_{p}$ of order $10^{-3}$ is in the small $E_{p}$ limit, for which the critical nucleation dimension and period at neutral stability are not that different from drained values. This is consistent with the idea that for geophysically relevant parameters dilatancy is not significant during transient slip nucleation, but becomes dominant as the slip speed increases.

[82] We conducted a limited number of tests to investigate whether (62) provides guidance for relating the maximum slip speeds in the two diffusion models. In the first example $E_{p}=3 \times 10^{-3}, a / b=0.9$, and $W / h_{d r}^{*}=16$; for this simulation $\log _{10}\left(v_{\max }\right)=-6.3$. For these parameters equation $(62)$ predicts equivalent behavior for $\mathcal{E U} \approx 0.06$. A membrane diffusion simulation with $\mathcal{E}=0.2$ and $\mathcal{U}=0.3$ yielded $\log _{10}\left(v_{\max }\right)=-6.45$. On the other hand, for $\mathcal{E}=2$ and $\mathcal{U}=$ 0.03 we found $\log _{10}\left(v_{\max }\right)=-7.4$. For a second comparison we took $E_{p}=3 \times 10^{-4}, a / b=0.9$ and $W / h_{d r}^{*}=12$, which yielded $\log _{10}\left(v_{\max }\right)=-4.0$. Equation (62) suggests equivalent slip speeds for $\mathcal{E}=0.2$, and $\mathcal{U} \approx 0.005$. A membrane diffusion simulation with these parameters yielded $\log _{10}\left(v_{\max }\right)=-4.4$. In summary, these comparisons are somewhat encouraging and indicate that a more comprehensive fracture mechanics based analysis could lead to an improved analytical understanding of the simulated slow slip events.

\section{Discussion}

\subsection{Comparison to Observations}

[83] Because this study did not consider depth-dependent material properties and stresses, including a transition to steady state velocity strengthening friction with depth, it is premature to associate particular parameters with field observations. Nevertheless, it is encouraging that there are parameters consistent with laboratory data that yield geophysically interesting behavior. Consider the observations detailed in the introduction. We have seen that simulations with $a / b=0.9,10^{-3.2} \leq E_{p} \leq 10^{-3}$, and $12 \leq W / h_{d r}^{*} \leq 20$, yield moment rates $\dot{M}_{\text {max }} / \dot{M}_{s s}$ of 10 to 100 and repeat periods of roughly 1 year. For these simulations the length of the slow slip zones are between 30 and $50 \mathrm{~km}$, roughly consistent with downdip dimensions in subduction zones. Note that for $\bar{\sigma}$ of the order of $1 \mathrm{MPa}$ and $d_{c}$ of order 10 to 100 microns, $h_{d r}^{*}$ is on the order of 0.4 to $4 \mathrm{~km}$. With dilatancy, $W / h_{d r}^{*}$ can be of the order 20 to 50 if not more. Low background effective stresses and dilatancy thus permit slow slip over appropriate spatial scales.

[84] For the parameters listed above the maximum slip in the simulations is on the order of $0.01 \mathrm{~m}$ and stress drops are on the order of $0.01 \mathrm{MPa}$, both consistent with geodetic observations. Along-strike propagation speeds of $\sim 10 \mathrm{~km} / \mathrm{d}$ have been observed geodetically. For the parameters stated above we observe updip propagation speeds of $\sim 2$ to $\sim 9 \mathrm{~km} / \mathrm{d}$ and downdip speeds of $\sim 9$ to $\sim 15 \mathrm{~km} / \mathrm{d}$, similar to observations. However, comparison of along-strike propagation speeds with updip two-dimensional model speeds should be viewed with caution. Once slip has extended the full updip width and begins propagating along strike, the minimum rupture zone dimension is fixed and the extending slip zone becomes highly elongate. This will decrease the energy release rate relative to a two-dimensional model, with consequent effects on the propagation speed. This may in fact help explain why slow slip does not accelerate as it propagates along strike. Details await fully three-dimensional calculations.

[85] In summary, while it is somewhat premature to identify particular parameter ranges with observations, it does appear that plausible material parameters, combined with very low background effective stresses can yield observed downdip slip zone dimensions, average slip amplitude, stress drop, average slip speeds, and recurrence times. Model propagation velocities in the dip direction are in the same range as observed along-strike; however, a full comparison awaits either observational determination of the updip velocity of slow slip events and three-dimensional model calculations.

\subsection{Summary of Evidence for High Pore Pressures}

[86] Observational evidence for high fluid pressures include high $v_{p} / v_{s}$ ratios inferred from tomographic [e.g., Kodaira et al., 2004; Shelly et al., 2006] and receiver function [Audet et al., 2009] studies. Audet et al. [2009] find that the subducted Juan de Fuca crust in the area where slow slip events are inferred to take place has Poisson's ratios in excess of 0.4 . This has been interpreted as indicating near lithostatic pore pressures, although the lack of appropriate laboratory data make it difficult to quantify this precisely. High fluid pressures are widely believed to result from dehydration reactions as the subducted slab passes through the stability field of a number of hydrous phases stable at low pressure and temperature conditions [e.g., Peacock et al., 2002; Liu and Rice, 2007]. If the overlying rocks 
have sufficiently low permeability, the source of fluid provided by dehydration reactions presumably maintains high ambient pore pressures. The low stress drops associated with slow slip events are also consistent with, but may not demand, low effective normal stresses.

[87] Modeling studies provide two additional arguments suggesting high ambient pore pressures. The first is that the critical nucleation dimension $h^{*}$ is inversely proportional to effective stress. Low effective stresses increase $h^{*}$ and thus help to rationalize the large dimensions of the stable slipping zones. Furthermore, we have shown that the efficacy of dilatant strengthening relative to frictional weakening, as measured by $\mathcal{E}$ and $E_{p}$ in the membrane and homogeneous models, respectively, increases with decreasing effective stress. Finally, we have shown that importance of dilatant strengthening relative to thermal pressurization weakening also scales inversely with effective normal stress. Thus, dilatancy is more likely to stabilize slip in areas with high ambient pore pressures and low effective normal stress.

\subsection{Consistency With Small Earthquakes}

[88] It is worthwhile considering whether or not the occurrence of very small earthquakes, at shallow depth, and thus modest normal stress, are in any way inconsistent with our inference that slow slip is favored by low effective stress. For example, consider small events at roughly $2 \mathrm{~km}$ depth near Parkfield, CA on the San Andreas fault. Stress measurements in the SAFOD pilot hole adjacent to the fault at $2 \mathrm{~km}$ depth show relatively high maximum compressive stress $\sim 120 \mathrm{MPa}$ at high angle $70^{\circ}$ to the fault [Hickman and Zoback, 2004], and hydrostatic pore pressures. From this we compute an effective normal stress of order $80 \mathrm{MPa}$. Assuming $a=0.015, a / b=0.8, d_{c}=50 \mu \mathrm{m}$, and $\mu=10^{4} M P a$, $h^{*}{ }_{d r}$ from equation (1) is of the order of $1.3 \mathrm{~m}$. According to (30) for fast slip to occur the membrane diffusion model, $\mathcal{E}$ must be less than 0.2 , and thus $f_{0} \epsilon / \beta b<17 \mathrm{MPa}$. Taking $f_{0}=0.6$ and $\beta \sim 8 \times 10^{-11} 1 / \mathrm{Pa}$, this requires $\epsilon<4 \times$ $10^{-5}$. The actual bound on $\epsilon$ is greater than this, because this analysis ignores thermal weakening. If the slip speeds get sufficiently high, thermal weakening may promote dynamic instability with parameters for which the isothermal analysis predicts that the undrained behavior is stable.

[89] For homogeneous diffusion we note from Figure 21 that for $E_{p}<10^{-4}$ the max average slip speeds are more than 4 orders of magnitude over the plate speed, at which point we suggest that thermal weakening effects dominate fault strength [Schmitt et al., 2007; Scmitt and Segall, 2008]. Using the same parameters, including $\bar{\sigma}=80 \mathrm{MPa}$, and assuming $c_{\text {hyd }} \sim 10^{-6} \mathrm{~m}^{2} / \mathrm{s}$ and $v^{\infty} \sim 10^{-9} \mathrm{~m} / \mathrm{s}$, we find $E_{p}<$ $10^{-4}$ requires $\epsilon h<10^{-6} \mathrm{~m}$. This easily encompasses range of $10^{-9}$ to $10^{-7} \mathrm{~m}$ estimated previously. Note also from equation (C18) that for $E_{p}<10^{-4}$ dilatancy increases $h^{*}$ by only about $1 \%$, so that the predictions are consistent with very small earthquake nucleation zones.

[90] Our inference is that effective stress is substantially lower in slow slip regions. We infer from Figure 21 that for $E_{p} \geq 10^{-3}$ the behavior is stable for a wide range of $W / h_{d r}^{*}$. This would be consistent with decreasing the effective normal stress by a factor of 10 to order $8 \mathrm{MPa}$. In conclusion there is no inconsistency between nucleating very small earthquakes at $2 \mathrm{~km}$ depth on the San Andreas fault and for dilatancy to stabilize slow slip events at much greater depth in subduction zones if the effective stress there is of the order of a few megapascals.

\section{Summary}

[91] 1 . With steady state velocity weakening friction $(a<b)$ on sufficiently long slip zones (relative to $h^{*}$ ), frictional weakening allows localized slip to nucleate. However, as slip accelerates it eventually becomes undrained and at this point dilatancy may act to suppress dynamic slip.

[92] 2. In the membrane diffusion approximation, when $\mathcal{E}$ exceeds $\mathcal{E}_{\text {crit }} \equiv 1-a / b$ a linearized stability analysis predicts that the critical nucleation length becomes infinite in the undrained limit. For $\mathcal{E}>\mathcal{E}_{\text {crit }}$ numerical simulations show that slip remains quasi-static over a very broad range of fault dimensions. In contrast, if $\mathcal{E}<\mathcal{E}_{\text {crit }}$ slip reaches radiation damping limits when the rupture dimension exceeds a critical value that is close to the predicted $h^{*}$ for membrane diffusion.

[93] 3. We have developed efficient numerical methods for coupling rate-state friction and dilatancy with elasticity and diffusion of pore fluid normal to the fault. This permits simulation of multiple episodic slow slip events, enabling conclusions to be drawn from simulations that minimize the dependence on arbitrary initial conditions.

[94] 4. We have developed equations for translating quantitatively between the membrane diffusion parameters $\mathcal{E}$ and $\mathcal{U}$ and the single isothermal homogeneous diffusion parameter $E_{p}$, both at neutral stability and (more approximately) at the fronts of propagating slow slip events. The membrane diffusion calculations are computationally more efficient, but because the equivalences near and far from steady state are not identical, a single set of membrane diffusion parameters seems unlikely to capture all aspects of the more time consuming homogeneous diffusion calculations.

[95] 5. For pore fluid diffusion into surroundings with homogeneous permeability, the ratio of dilatant strengthening to thermal weakening scales with $\epsilon h / d_{c}, \rho c / \Lambda$, and $1 / f_{0} \beta\left(\sigma-p^{\infty}\right)$, where $h$ is the shear zone thickness, and $\Lambda$ is the ratio of thermal expansivity to compressibility. High pore pressure thus mitigates against frictional and thermal weakening and favors slow slip, consistent with seismic observations of anomalous $v_{p} / v_{s}$. Whether slip is ultimately slow or fast may depend on whether dilatancy prevents slip speeds from reaching rates at which thermal pressurization dominates.

[96] 6. In isothermal calculations with $a / b=0.9$ and $10^{-3.2} \leq E_{p} \leq 10^{-3}$, for $12 \leq W / h_{d r}^{*} \leq 20$, and perhaps longer, average transient slip rates are on the order of 10 to 100 times plate velocity, and repeat times are on the order of 1 year, comparable to that observed in Cascadia. For these parameters maximum slip is $\sim 0.01 \mathrm{~m}$ and stress drops are $\sim 0.01 \mathrm{MPa}$, both consistent with geodetic observations. Model propagation speeds in the dip direction are in the same range as observed along-strike. It is possible that similar behavior can be obtained for a somewhat broader range of parameters, but we have not explored this fully.

[97] 7. For a broad range of parameters simulations exhibit slow phases driven by the downdip, constant velocity boundary condition, and faster (but quasi-static) phases that relax the accumulated stress. The faster phases are assumed 
to model geodetically observable slow slip events, while the slow phases may help to explain tremor observed between strong ETS events.

[98] 8. Slow slip accommodates only a fraction of the net relative plate motion, implying that the remaining deficit is made up during coseismic or rapid postseismic slip.

\section{Appendix A: Frequency of Oscillations at Neutral Stability for Membrane Diffusion}

[99] From Segall and Rice [1995, equation (A5)] we deduce that the frequency of oscillations at neutral stability for the isothermal membrane diffusion model is given by

$$
\omega=\frac{v^{\infty}}{d_{c}} \mathcal{U}^{-1} \sqrt{\frac{F}{1-F}}
$$

where $F=F(\mathcal{E}, \mathcal{U}, a / b)$ is given by (27). The period of oscillation, $T$, normalized by $d_{c} / v^{\infty}$ is thus

$$
\frac{T v^{\infty}}{d_{c}}=\frac{\delta_{n s}}{d_{c}}=2 \pi \mathcal{U} \sqrt{\frac{1-F}{F}} .
$$

Here, $\delta_{n s}=T v^{\infty}$ is the slip per period at neutral stability. In the drained limit $\mathcal{U} \rightarrow 0, F$ tends to $[(b-a) / b] \mathcal{U}^{2}$, such that for drained deformation $\delta_{n s} / d_{c}=2 \pi \sqrt{a /(b-a)}$ [Ruina, 1983].

[100] In order to determine the asymptotic behavior in the undrained limit, take Taylor series expansions for $\lambda$ and $\gamma$ (from equation (27)) in the limit $1 / \mathcal{U} \rightarrow 0$. There are three cases of interest, corresponding to $\mathcal{E}>1-a / b, \mathcal{E}=1-a / b$, and $\mathcal{E}<1-a / b$. The results are

$$
\begin{aligned}
\frac{T v^{\infty}}{d_{c}} & =2 \pi \mathcal{U} \sqrt{\frac{\mathcal{E}-(1-a / b)}{(1-a / b)}} \quad \mathcal{E}>1-\frac{a}{b} \\
& =\frac{2 \pi}{\sqrt{2}} \mathcal{U}^{1 / 2} \sqrt{\sqrt{\frac{4 a / b}{1-a / b}+1}+1} \quad \mathcal{E}=1-\frac{a}{b} \\
& =2 \pi \sqrt{\frac{a / b}{1-a / b-\mathcal{E}}} \quad \mathcal{E}<1-\frac{a}{b}
\end{aligned}
$$

Notice that for $\mathcal{E}>\mathcal{E}_{\text {crit }}$ that the period increases linearly with $\mathcal{U}$, whereas for $\mathcal{E}=\mathcal{E}_{\text {crit }}$, the period increases with $\sqrt{\mathcal{U}}$, and for $\mathcal{E}$ less than critical the period asymptotes to a constant value, independent of $\mathcal{U}$.

[101] In the drained limit $\mathcal{U} \rightarrow 0, \gamma \rightarrow 0$ and we find that

$$
k_{m d} \approx 1-\frac{\mathcal{E} \mathcal{U}^{2}}{\mathcal{U E}+\frac{a}{b}} \approx 1-\mathcal{E} \mathcal{U}^{2} \frac{b}{a},
$$

the latter accurate if $\mathcal{E U} \ll a / b$. This leads to a critical nucleation dimension

$$
\frac{h_{m d}^{*}}{h_{d r}^{*}}-1 \approx \mathcal{E} \mathcal{U}^{2} \frac{b}{a}
$$

Similarly the dimensionless period in the drained limit is

$$
\begin{aligned}
& \frac{T_{m d}}{T_{d r}} \approx \sqrt{1+\frac{b}{a} \mathcal{E U}} \\
& \frac{T_{m d}}{T_{d r}}-1 \approx \frac{1}{2} \frac{b}{a} \mathcal{E} \mathcal{U} .
\end{aligned}
$$

\section{Appendix B: Finite Difference Procedure}

[102] The system of equations to be solved for the homogeneous diffusion problem are (16), (3), and (11).

$$
\begin{gathered}
\dot{v}(x, t)=f(v(x, t), \theta(x, t), \dot{\theta}(x, t), p(x, 0, t), \dot{p}(x, 0, t)) \\
\text { on } y=0 \\
\dot{\theta}(x, t)=h(v(x, t), \theta(x, t)) \quad \text { on } y=0 \\
\dot{p}(x, y, t)=c_{h y d} p(x, y, t)_{y y} \quad \text { on } y>0
\end{gathered}
$$

where $p_{y y}$ indicates the second spatial derivative of $\mathrm{p}$. The boundary conditions on pore pressure are

$$
\begin{aligned}
p(x, 0, t)_{y}= & g(\theta(x, t), \dot{\theta}(x, t)) \quad \text { on } y=0 \\
& \lim _{y \rightarrow \infty} p(x, y, t)=p_{\infty} .
\end{aligned}
$$

In this work we differentiate the momentum balance on the fault (B1), so the problem is cast as a system of differential equations. Integrating over long periods of time inevitably lead to numerical error such that the original momentum balance (6) will no longer be satisfied. However, for the simulations reported here, the differential equation formulation is sufficiently accurate.

[103] The first two equations are not involved in the finite difference calculation, therefore for notational simplicity we write $\theta$ and $v$ together as a single variable $u=[v, \theta, \dot{\theta}]$, such that (B1) and (B4) become:

$$
\begin{array}{ll}
\dot{u}(x, t)=f(u(x, t), p(x, 0, t), \dot{p}(x, 0, t)) & \text { on } y=0 \\
p(x, 0, t)_{y}=g(u(x, t)) & \text { on } y=0
\end{array}
$$

\section{B1. Discretization in the $y$ Direction}

[104] We make the domain $\left[0, y_{\infty}\right]$ sufficiently large that $p\left(y_{\infty}\right) \approx p_{\infty}$. Thus, we approximate (B5) by $p\left(x, y_{\infty}, t\right)=p_{\infty}$. Near the fault it is important that the discretization be sufficiently fine to capture the steep gradient in $p$. To achieve this we make the following change of coordinate between $y$ and $z$ :

$$
z(y)=\ln (c+y) \quad \text { or, equivalently, } \quad y(z)=-c+e^{z} .
$$

This change of coordinate has the effect of making the mesh dense near $y=0$ and sparse near $y=y_{\infty}$. We have found 
that $c=10^{-2}$ yields good results. Following the change of coordinate, the system of equations is

$$
\begin{array}{cc}
\dot{u}=f(u, p, \dot{p}) & \text { on } y=0 \\
\dot{p}=c_{\text {hyd }} e^{-z}\left(e^{-z} p_{z}\right)_{z} & \text { on } y>0 \\
e^{-z} p_{z}=g(u) & \text { on } y=0 \\
p=p_{\infty} & \text { on } y=y_{\infty} .
\end{array}
$$

We discretize the PDE (B9) and boundary conditions (B10) and (B11) in space, letting $\delta=\Delta z / 2$ :

$$
\begin{gathered}
\dot{p}_{k}=c_{h y d} e^{-z_{k}} \times\left(\frac{-e^{-\left(z_{k}-\delta\right)}\left(p_{k}-p_{k-1}\right)+e^{-\left(z_{k}+\delta\right)}\left(p_{k+1}-p_{k}\right)}{\Delta z^{2}}\right) \\
e^{-z_{0}} \frac{p_{1}-p_{-1}}{2 \Delta z}=g \quad \text { and } \quad p_{K}=p_{\infty}
\end{gathered}
$$

for $k=0,1, \ldots, K$. The discretization (B12) is a second-orderaccurate conservative discretization of the gradient of the flux function $e^{-z} p_{z}$ in (B9). The discretization of the Neumann boundary condition (B13) is a second-order-accurate approximation centered around $k=0$. Note that the ghost variable $p_{-1}$ is eliminated when (B13) is introduced into (B12).

\section{B2. Implicit-Explicit Time Stepping}

[105] We next consider the method for time stepping the system of equations. Let $p_{k m}^{n}$ be the value of $p$ at the $k$ th point in the $y$ direction and the $m$ th point in the $x$ direction, at the $n$th time step. For simplicity, we illustrate the approach with the first-order in time Euler's method; however, the actual code uses higher-order time stepping for the nondiffusion variables. Also for simplicity in presentation we illustrate the time stepping procedure for the spatially uniform, rather than log discretization. Equations (B7), (B6), and (B3) are discretized in time as:

$$
\begin{aligned}
& \frac{u_{m}^{n+1}-u_{m}^{n}}{\Delta t}=f\left(u_{m}^{n}, p_{0 m}^{n}, \frac{p_{0 m}^{n}-p_{0 m}^{n-1}}{\Delta t}\right) \\
& \frac{p_{k m}^{n+1}-p_{k m}^{n}}{\Delta t}=c_{h y d}\left(\frac{p_{(k-1) m}^{n+1}-2 p_{k m}^{n+1}+p_{(k+1) m}^{n+1}}{\Delta y^{2}}\right) \\
& \frac{p_{1 m}^{n+1}-p_{(-1) m}^{n+1}}{2 \Delta y}=g\left(u_{m}^{n+1}\right) \quad \text { and } \quad p_{K m}^{n+1}=p_{\infty} .
\end{aligned}
$$

where $m=1, \ldots, M$ and $k=1, \ldots, K$. Note that the first equation for $u_{m}$ is explicit, whereas the equations for $p$ are implicit in that pore pressure at time step $n+1$ depends on $p^{n+1}$, and $u_{m}^{n+1}$. An important feature is that for each position along the fault (indexed by $m$ ), the pore pressure along the fault normal profile depends only on the quantities at index $m$. Thus, the pore pressures on the fault are only coupled through the friction/elasticity equations (first set of equations above). Thus, the finite difference computations in $y$ decouple, such that $M$ small systems of equations (with $K$ elements) are solved at each time step, rather than one very large system of equa- tions. This is vastly more efficient that solving the full implicit equations.

[106] Our code integrates in time using the explicit RungeKutta $(2,3)$ scheme implemented in Matlab's ode23.

\section{Appendix C: Linearized Stability Analysis for Homogeneous Diffusion}

[107] Our approach follows Segall and Rice [1995] with homogeneous diffusion replacing membrane diffusion. Adopting the normalization in section 2.1, the linearized equations perturbed around steady state [Segall and Rice, 1995, equations (23a)-(23e)] become

$$
\begin{gathered}
\frac{a}{b} \Delta \dot{\tilde{v}}=-\Delta \dot{\tilde{\theta}}+\frac{f_{0} \Delta \dot{\tilde{p}}}{b}-\tilde{k}\left(1-\frac{a}{b}\right) \Delta \tilde{v} \\
\Delta \dot{\tilde{\theta}}=-\Delta \tilde{\theta}-\Delta \tilde{v} \\
\Delta \dot{\tilde{\phi}}=\Delta \tilde{\phi}+\epsilon \Delta \tilde{v} .
\end{gathered}
$$

In the following we drop the tildes with the understanding that all variables are dimensionless, unless otherwise specified. The normalized diffusion equation is given by (13). Linearizing $\theta$ around steady state, $\theta=\theta_{s s}+\Delta \theta=1+\Delta \theta$, the boundary condition in (13) becomes

$$
\left.\frac{\partial p}{\partial y}\right|_{y=0}=-E_{p} \Delta \dot{\theta} .
$$

We seek solutions to the linearized equations of the form $\Delta \theta=\Theta e^{s t}, \Delta v=V e^{s t}, \Delta \phi=\Phi e^{s t}$. For solutions of this form (C4) becomes

$$
\left.\frac{\partial p}{\partial y}\right|_{y=0}=-E_{p} s \Theta e^{s t} .
$$

The solution to the diffusion equation with boundary condition (C5) and the constraint that $p=p^{\infty}$ at $y=\infty$ is

$$
p=E_{p} s^{1 / 2} \Theta e^{s t} e^{-\sqrt{s y}}+p^{\infty} .
$$

The rate of pore pressure change on the fault is thus

$$
\left.\dot{p}\right|_{y=0}=E_{p} s^{3 / 2} \Theta e^{s t} .
$$

Thus, the appropriate linearized form of $\Delta \dot{p}$ in $(\mathrm{C} 1)$ is given by (C7). Combining with (C1)-(C3) and $\Delta \theta=\Theta e^{s t}$, $\Delta v=V e^{s t}$ leads to the following equation for $s$

$$
\frac{a}{b} s^{2}+\frac{f_{0} E_{p}}{b} s^{3 / 2}+(k-1)\left(1-\frac{a}{b}\right) s+k\left(1-\frac{a}{b}\right)=0 .
$$

Whether slip is stable or not depends on the real part of $s$. We first establish that in the limit $k \rightarrow \infty, \Re[s]<0$. Note that if $s$ is of order $k$, then in the limit $s=-k(b / a-1)$, whereas if $s$ is of order unity, then in the limit $s=-1$. Thus, for a sufficiently stiff system, $k \rightarrow \infty$, small perturbations decay. Also note that for nonzero $k$ there is no solution at $s=0$. We thus assume that as $k$ decreases the first root to cross to the positive real half plane does so at $s=i \omega$, implying purely harmonic oscillations at $k=k_{c r i t}$. 
Substituting $s=i \omega$ into (C8) and separating real and imaginary parts leads to

$$
\begin{gathered}
k_{c r i t}=\frac{a \omega^{2}}{(b-a)}+\frac{f_{0} E_{p}}{\sqrt{2}(b-a)} \omega^{3 / 2} \\
k_{\text {crit }}=1-\frac{f_{0} E_{p}}{\sqrt{2}(b-a)} \omega^{1 / 2} .
\end{gathered}
$$

In the drained limit, $E_{p}=0$, equation (C10) becomes $k_{\text {crit }}=1$, whereas (C9) leads to $\omega=(b / a-1)^{1 / 2}$, recovering the fully drained limit. Equating (C9) and (C10),

$$
\frac{a}{b} \omega^{2}+\frac{f_{0} E_{p}}{\sqrt{2} b} \omega^{1 / 2}(\omega+1)-\left(1-\frac{a}{b}\right)=0
$$

a quartic equation for $\omega^{1 / 2}$. Rearranging,

$$
\frac{f_{0} E_{p}}{\sqrt{2} b}=\frac{1-(a / b)\left(1+\omega^{2}\right)}{\omega^{1 / 2}(\omega+1)} .
$$

For a specified $a / b$ and $\omega$ one can compute the corresponding value of $f_{0} E_{p} / b$. This and the assumed $\omega$ can be combined with (C10) to give the departure of $k_{\text {crit }}$ from its drained value. The variation in $k_{c r i t}$ and $\omega$ as a function of $f_{0} E_{p} / b$ is given in Figure 22.

\section{C1. Limiting Behavior for Small $\boldsymbol{E}_{\boldsymbol{p}}$}

[108] We can obtain useful asymptotic results in the limits of small and large $E_{p}$. For small $E_{p}$, write

$$
\omega^{1 / 2}=\left(\frac{b}{a}-1\right)^{1 / 4}+\Delta,
$$

where $\Delta$ represents the deviation from the drained, $E_{p}=0$, result. Substituting this into $(\mathrm{C} 11)$ and retaining only the lowest-order terms in $\Delta$ and $E_{p}$ leads to

$$
\begin{gathered}
\Delta \approx-\frac{f_{0} E_{p}}{\sqrt{2} b} \frac{(b / a-1)^{1 / 2}+1}{4(a / b)(b / a-1)^{1 / 2}} ; \\
\frac{\omega}{\omega_{d r}} \approx 1-\frac{f_{0} E_{p}}{2 \sqrt{2} b} \frac{\left(\frac{b}{a}-1\right)^{3 / 4}+\left(\frac{b}{a}-1\right)^{1 / 4}}{1-\frac{a}{b}} ;
\end{gathered}
$$

where we have made use of $\omega_{d r}=(b / a-1)^{1 / 2}$. The period of oscillations at neutral stability is inversely proportional to the frequency, $T / T_{d r}=\left(\omega / \omega_{d r}\right)^{-1}$

$$
\frac{T}{T_{d r}}-1 \approx \frac{f_{0} E_{p}}{2 \sqrt{2} b} \frac{\left(\frac{b}{a}-1\right)^{3 / 4}+\left(\frac{b}{a}-1\right)^{1 / 4}}{\left(1-\frac{a}{b}\right)} .
$$

Substituting (C13) and (C14) into (C10) and neglecting the $E_{p} \Delta$ term,

$$
\frac{k_{\text {crit }}}{k_{\text {crit_dr }}} \approx 1-\frac{f_{0} E_{p}}{\sqrt{2} b} \frac{\left(\frac{b}{a}-1\right)^{1 / 4}}{\left(1-\frac{a}{b}\right)} .
$$

The critical nucleation dimension is inversely proportional to the critical stiffness, such that

$$
\frac{h^{*}}{h_{d r}^{*}}-1 \approx \frac{f_{0} E_{p}}{\sqrt{2} b} \frac{\left(\frac{b}{a}-1\right)^{1 / 4}}{\left(1-\frac{a}{b}\right)} .
$$

The limiting behavior for small $E_{p},(\mathrm{C} 16)$ and $(\mathrm{C} 18)$, are compared to the complete results in Figure 22.

\section{C2. Limiting Behavior for Large $E_{p}$}

[109] In the limit that $E_{p} \rightarrow \infty$ we find by inspection of (C11) that $\omega$ tends to zero and

$$
\begin{gathered}
\frac{f_{0} E_{p}}{\sqrt{2} b} \omega^{1 / 2} \rightarrow 1-\frac{a}{b} ; \\
\omega \approx\left(1-\frac{a}{b}\right)^{2}\left(\frac{f_{0} E_{p}}{\sqrt{2} b}\right)^{-2} .
\end{gathered}
$$

[110] The reciprocal of (C20) leads to the large $E_{p}$ period at neutral stability

$$
T \approx 2 \pi\left(1-\frac{a}{b}\right)^{-2}\left(\frac{f_{0} E_{p}}{\sqrt{2} b}\right)^{2} .
$$

Substituting (C19) into (C10) shows only that $k_{\text {crit }} \rightarrow 0$ as $E_{p} \rightarrow \infty$. To proceed further we write

$$
\omega^{1 / 2}=\left(1-\frac{a}{b}\right)\left(\frac{f_{0} E_{p}}{\sqrt{2} b}\right)^{-1}+\Delta,
$$

where $\Delta$ now represents the deviation from the limiting behavior in (C19). Substituting (C22) into (C11) and retaining only the lowest-order terms in $\Delta$ and $1 / E_{p}$ leads to

$$
\Delta \approx-\left(1-\frac{a}{b}\right)^{3}\left(\frac{f_{0} E_{p}}{\sqrt{2} b}\right)^{-3} .
$$

Substituting (C22) and (C23) into (C10), we obtain

$$
\frac{k_{\text {crit }}}{k_{\text {crit_dr }}} \approx\left(1-\frac{a}{b}\right)^{2}\left(\frac{f_{0} E_{p}}{\sqrt{2} b}\right)^{-2}
$$

such that

$$
\frac{h^{*}}{h_{d r}^{*}} \approx\left(1-\frac{a}{b}\right)^{-2}\left(\frac{f_{0} E_{p}}{\sqrt{2} b}\right)^{2} .
$$

The limiting behavior for high $E_{p},(\mathrm{C} 21)$ and (C25), are compared to the complete results in Figure 22.

\section{Appendix D: Analytical Solutions for Pore Pressure Change}

[111] We consider here analytical results for the pore pressure response to a step change in slip speed for both the 
membrane diffusion and homogeneous diffusion models. For a step change in slip speed $v(t)=v H(t)$, the slip law (3) can be integrated exactly to yield

$$
\theta=\theta_{f}\left(\frac{\theta_{i}}{\theta_{f}}\right)^{\exp \left(-\delta / d_{c}\right)}
$$

where $\delta=v t$ is the accumulated slip, and $\theta_{i}$ and $\theta_{f}=d_{c} / v$ are the initial and final states, respectively. Thus,

$$
\ln \left(\frac{v \theta}{d_{c}}\right)=\ln \left(\frac{v \theta_{i}}{d_{c}}\right) e^{-\delta / d_{c}}
$$

Also, for the slip law

$$
\frac{\dot{\theta}}{\theta}=-\frac{v}{d_{c}} \ln \left(\frac{v \theta}{d_{c}}\right)
$$

\section{D1. Membrane Diffusion}

[112] Combining the membrane diffusion equation (15) with the constitutive law for dilatancy (5) and the slip law (3b) yields

$$
\frac{d \Delta p}{d t}+\frac{\Delta p}{t_{f}}=\frac{\epsilon}{\beta} \frac{\dot{\theta}}{\theta}
$$

where $\Delta p$ is the difference in pore pressure relative to the far-field value. The differential equation (D4) is solved for a step in slip speed is at time $t=0$, by use of an integrating factor $\exp \left(t / t_{f}\right)$, which yields

$$
\begin{aligned}
\Delta p(t) & =-\frac{\epsilon}{\beta} \ln \left(\frac{v \theta_{i}}{d_{c}}\right) \frac{v t_{f}}{d_{c}-v t_{f}}\left(e^{-v t / d_{c}}-e^{-t / t_{f}}\right) ; \frac{v t_{f}}{d_{c}} \neq 1 \\
& =-\frac{\epsilon}{\beta} \ln \left(\frac{v \theta_{i}}{d_{c}}\right) \frac{v t}{d_{c}} e^{-v t / d_{c}} ; \quad \frac{v t_{f}}{d_{c}}=1 .
\end{aligned}
$$

\section{D2. Homogeneous Diffusion}

[113] For this imposed slip history, the isothermal diffusion equation (11) can be solved exactly. The governing equation is

$$
\frac{\partial p}{\partial t}-c_{h y d} \frac{\partial^{2} p}{\partial y^{2}}=0
$$

with boundary conditions

$$
\left.\frac{\partial p}{\partial y}\right|_{y=0}=\frac{h \dot{\phi}}{2 \beta c_{h y d}}=\frac{\epsilon h}{2 \beta c_{h y d}} \frac{\dot{\theta}}{\theta} .
$$

The initial conditions are that the pore pressure is everywhere zero. We seek a solution for a step change in $v$ at $t=0$ with associated change in $\phi$. Combining equations (D7), (D3), and the result (D2) for a step change in slip speed, yields the modified boundary condition

$$
\left.\frac{\partial p}{\partial y}\right|_{y=0}=\frac{\epsilon h}{2 \beta c_{\text {hyd }}}\left(\frac{v}{d_{c}}\right) \ln \left(\frac{v \theta_{i}}{d_{c}}\right) e^{-v t / d_{c}} .
$$

Take the Laplace transform of the differential equation (D6) and boundary condition (D8), which yields

$$
\begin{aligned}
\hat{p}-\frac{c_{h y d}}{s} \frac{d^{2} \hat{p}}{d y^{2}} & =0 \\
\left.\frac{\partial \hat{p}}{\partial y}\right|_{y=0} & =\frac{\Psi(v)}{s+v / d_{c}}
\end{aligned}
$$

where $s$ is the transform variable, ${ }^{\wedge}$ indicates a transformed variable, and

$$
\Psi(v)=\frac{\epsilon h}{2 \beta c_{\text {hyd }}}\left(\frac{v}{d_{c}}\right) \ln \left(\frac{v \theta_{i}}{d_{c}}\right) .
$$

The solutions to the differential equation (D9) are

$$
\hat{p}=A e^{ \pm \sqrt{s / c_{\text {hyd }}} y} .
$$

We retain only the decaying solution. The constant $A=$ $\hat{p}(0, s)$ gives the pore pressure on the fault. From the boundary condition in (D9)

$$
\hat{p}(y=0, s)=A=-\Psi(v) \frac{\sqrt{c_{h y d} / s}}{s+v / d_{c}} .
$$

Note that the inverse transform of $1 / \sqrt{s}$ is $1 / \sqrt{\pi t}$, while the inverse transform of $1 /\left(s+v / d_{c}\right)$ is $\exp \left(-v t / d_{c}\right)$. Thus, from the convolution theorem we have

$$
p(y=0, t)=-\Psi(v) \sqrt{\frac{c_{h y d}}{\pi}} \int_{0}^{t^{\prime}} \frac{1}{\sqrt{t^{\prime}}} e^{-\frac{v}{d_{c}}\left(t-t^{\prime}\right)} d t^{\prime} .
$$

A change of variables $z^{2}=v t^{\prime} / d_{c}$ leads to

$$
\begin{gathered}
p(y=0, t)=-2 \Psi(v) \sqrt{\frac{c_{\text {hyd }} d_{c}}{\pi v}} D\left(\sqrt{\frac{\delta}{d_{c}}}\right) \\
-\frac{2 E_{p}}{\sqrt{\pi}}\left(\sigma-p^{\infty}\right) \sqrt{\frac{v}{v^{\infty}}} \ln \left(\frac{v \theta_{i}}{d_{c}}\right) D\left(\sqrt{\frac{\delta}{d_{c}}}\right),
\end{gathered}
$$

where $D(z)$ is known as Dawson's Integral, see equation (53).

\section{Notation}

$a, b$ Rate-state friction coefficients.

$c$ Specific heat capacity.

$c_{t h}$ Thermal diffusivity.

$c_{\text {hyd }}$ Hydraulic diffusivity.

$d_{c}$ Critical slip weakening distance.

$\mathcal{E}$ Nondimensional dilatancy, membrane diff. equation (22).

$\mathcal{E}_{\text {crit }}$ Critical $\mathcal{E}$, equation (30).

$E_{p}$ Nondimensional dilatancy efficiency, equation (13).

$E_{T}$ Nondimensional shear-heating efficiency, equation (12).

$f$ Coefficient of friction, $f_{0}$ nominal value.

$G_{c}$ Fracture energy.

$h$ Thickness of shear zone. 
$h_{w}$ Thickness of wall zone.

$h^{*}$ Critical nucleation dimension.

$h_{d r}^{*}$ Drained critical nucleation dimension, equation (1).

$k_{c r i t}$ Critical spring stiffness.

$\tilde{K}_{c r i t}$ Nondimensional critical spring stiffness.

$p$ Pore pressure.

$p^{\infty}$ Remote pore pressure.

$t$ Time.

$t_{f}$ Characteristic time for fluid diffusion.

$\mathcal{T}$ Temperature.

$T$ Period of oscillations at neutral stability.

$T_{h d}$ Period at neutral stability, homogeneous diffusion.

$\mathcal{U}$ Nondimensional drainage time, membrane diff. equation (23).

$v$ Fault slip speed.

$v_{s s}$ Steady state slip speed.

$v^{\infty}$ Rate of plate motion.

$v_{s}$ Shear wave velocity.

$W$ Width of velocity weakening fault in $x$ direction.

$x$ Fault parallel distance.

$y$ Fault perpendicular distance.

$\beta$ Compressibility, fluid plus pore.

$\delta$ Fault slip.

$\delta^{*}$ Effective slip weakening distance.

$\varepsilon$ Dilatancy parameter.

$\gamma$ Shear strain.

$\kappa$ Permeability.

$\Lambda$ Thermal pressurization factor.

$\phi$ Porosity.

$\mu$ Shear modulus.

$\nu$ Poisson's ratio.

$\eta$ Pore fluid viscosity.

$\rho$ Rock mass density.

$\sigma$ Fault normal stress.

$\bar{\sigma}$ Effective normal stress.

$\tau$ Shear stress.

$\theta$ Friction state variable.

$\theta_{i}$ State variable prior to velocity step.

[114] Acknowledgments. Jessica Hawthorne helped in the linear stability analysis for homogeneous diffusion. We gratefully acknowledge support from the National Science Foundation (EAR-0838267), the U.S. Geological Survey (08HQGR0014, P.S., and 08HQGR0047, A.M.R.), and the Southern California Earthquake Center. SCEC is funded by NSF Cooperative Agreement EAR-0106924 and USGS Cooperative Agreement 02HQAG0008. This paper is SCEC contribution 1362.

\section{References}

Ampuero, J.-P., and A. M. Rubin (2008), Earthquake nucleation on rate and state faults: Aging and slip laws, J. Geophys. Res., 113, B01302, doi:10.1029/2007JB005082

Audet, P., M. G. Bostock, N. I. Christensen, and S. M. Peacock (2009), Seismic evidence for overpressured subducted oceanic crust and megathrust fault sealing, Nature, 457, 76-78, doi:10.1038/nature07650.

Bayart, E., A. M. Rubin, and C. Marone (2006), Evolution of fault friction following large velocity jumps, Eos Trans. AGU, 87(52), Fall Meet. Suppl., Abstract S31A-0180.

Beeler, N. M., T. E. Tullis, and J. D. Weeks (1994), The roles of time and displacement in the evolution effect in rock friction, Geophys. Res. Lett., 21(18), 1987-1990

Brooks, B. A., J. H. Foster, M. Bevis, L. N. Frazer, C. J. Wolfe, and M. Behn (2006), Periodic slow earthquakes on the flank of Kilauea volcano, Hawai'i, Earth Planet. Sci. Lett., 246(3-4), 207-216.

Brown, J. R., G. C. Beroza, S. Ide, K. Ohta, D. R. Shelly, S. Y. Schwartz, W. Rabbel, M. Thorwart, and H. Kao (2009), Deep low-frequency earthquakes in tremor localize to the plate interface in multiple subduction zones, Geophys. Res. Lett., 36, L19306, doi:10.1029/2009GL040027.
Brudzinski, M. R., and R. M. Allen (2007), Segmentation in episodic tremor and slip all along Cascadia, Geology, 35, 907-910.

Cervelli, P., P. Segall, K. Johnson, M. Lisowski, and A. Miklius (2002), Sudden aseismic fault slip on the south flank of Kilauea volcano, Nature, 415, 1014-1018.

Delahaye, E. J., J. Townend, M. E. Reyners, and G. Rogers (2009), Microseismicity but no tremor accompanying slow slip in the Hikurangi subduction zone, New Zealand, Earth Planet. Sci. Lett., 277, 21-28.

Dieterich, J. H., and B. D. Kilgore (1994), Direct observation of frictional contacts: New insights for state-dependent properties, Pure. Appl. Geophys., 143, 283-302.

Douglas, A., J. Beavan, L. Wallace, and J. Townend (2005), Slow slip on the northern Hikurangi subduction interface, New Zealand, Geophys. Res. Lett., 32, L16305, doi:10.1029/2005GL023607.

Dragert, H., K. Wang, and T. S. James (2001), A silent slip event on the deeper Cascadia subduction interface, Science, 292, 1525-1528.

Hickman, S., and M. Zoback (2004), Stress orientations and magnitudes in the SAFOD pilot hole, Geophys. Res. Lett., 31, L15S12, doi:10.1029/ 2004GL020043.

Hillers, G., and S. A. Miller (2006), Stability regimes of a dilatant, fluidinfiltrated fault plane in a three-dimensional elastic solid, J. Geophys. Res., 111, B08304, doi:10.1029/2005JB003872.

Hirose, H., K. Hirahara, F. Kimata, N. Fujii, and S. Miyazaki (1999), A slow thrust slip event following the two 1996 Hyuganada eartqhuakes beneath the Bungo Channel, southwest Japan, Geophys. Res. Lett., 26, 3237-3240.

Ide, S., D. R. Shelly, and G. C. Beroza (2007), Mechanism of deep low frequency earthquakes: Further evidence that deep non-volcanic tremor is generated by shear slip on the plate interface, Geophys. Res. Lett., 34 , L03308, doi:10.1029/2006GL028890.

Kao, H., S.-J. Shan, H. Dragert, G. Rogers, J. F. Cassidy, and K. Ramachandran (2005), A wide depth distribution of seismic tremors along the northern Cascadia margin, Nature, 436, 841-844.

Kilgore, B. D., J. H. Dieterich, and M. L. Blanpied (1993), Velocity dependent friction of granite over a wide range of conditions, Geophys. Res. Lett., 20, 903-906

Kodaira, S., T. Iidaka, A. Kato, J. O. Park, T. Iwasaki, and Y. Kaneda (2004), High pore fluid pressure may cause silent slip in the Nankai trough, Science, 304, 1295-1298, doi:10.1126/science.1096535.

Kostoglodov, V., S. K. Singh, J. A. Santiago, K. M. Larson, A. R. Lowry, and R. Bilham (2003), A large silent earthquake in the Guerrero seismic gap, Mexico, Geophys. Res. Lett., 30(15), 1807, doi:10.1029/2003GL017219.

Kuroki, H., H. M. Ito, H. Takayama, and A. Yoshida (2004), 3-D simulation of the occurrence of slow slip events in the Tokai region with a rate- and state-dependent friction law, Bull. Seismol. Soc. Am., 94(6), 2037-2050.

Lachenbruch, A. H. (1980), Frictional heating, fluid pressure, and the resistance to fault motion, J. Geophys. Res., 85, 6097-6112.

Lapusta, N., J. R. Rice, Y. Ben-Zion, and G. Zheng (2000), Elastodynamic analysis for slow tectonic loading with spontaneous rupture episodes on faults with rate- and state-dependent friction, J. Geophys. Res., 105(B10), 23,765-23,789, doi:10.1029/2000JB900250.

La Rocca, M., K. C. Creager, D. Galluzzo, S. Malone, J. E. Vidale, J. R. Sweet, and A. G. Wech (2009), Cascadia tremor located near plate interface constrained by $S$ minus $P$ wave times, Science, 323, 620-623.

Larson, K. M., V. Kostoglodov, S. Miyazaki, and J. A. S. Santiago (2007), The 2006 aseismic slow slip event in Guerrero, Mexico: New results from GPS, Geophys. Res. Lett., 34, L13309, doi:10.1029/2007GL029912.

Linde, A. T., M. T. Gladwin, M. J. S. Johnston, R. L. Gwyther, and R. G. Bilham (1996), A slow earthquake sequence on the San Andreas Fault, Nature, 383, 65-68.

Liu, Y., and J. R. Rice (2005a), Aseismic slip transients emerge spontaneously in three-dimensional rate and state modeling of subduction earthquake sequences, J. Geophys. Res., 110, B08307, doi:10.1029/ 2004JB003424.

Liu, Y., and J. R. Rice (2005b), Pore pressure evolution in shallow subduction earthquake sequences and effects on aseismic slip transients: Numerical modeling with rate and state friction, Eos Trans. AGU, 86(52), Fall Meet. Suppl., Abstract T11E-05.

Liu, Y., and J. R. Rice (2007), Spontaneous and triggered aseismic deformation transients in a subduction fault model, J. Geophys. Res., 112, B09404, doi:10.1029/2007JB004930.

Liu, Y., and J. R. Rice (2009), Slow slip predictions based on granite and gabbro friction data compared to GPS measurements in northern Cascadia, J. Geophys. Res., 114, B09407, doi:10.1029/2008JB006142.

Liu, Y., and A. M. Rubin (2010), Role of fault gouge dilatancy on aseismic deformation transients, J. Geophys. Res., 115, B10414, doi:10.1029/ 2010JB007522.

Lockner, D. A., H. Naka, H. Tanaka, H. Ito, and R. Ikeda (2000), Permeability and strength of core samples from the Nojima Fault of the 1995 
Kobe earthquake, in Proceedings of the International Workshop on the Nojima Fault Core and Borehole Data Analysis, Tsukuba Japan, 22-23 November, 1999, edited by H. Ito et al., U.S. Geol. Surv. Open File Rep., 00-129, 147-152.

Lohman, R. B., and J. J. McGuire (2007), Earthquake swarms driven by aseismic creep in the Salton Trough, California, J. Geophys. Res., 112, B04405, doi:10.1029/2006JB004596.

Marone, C. (1998), Laboratory-derived friction laws and their application to seismic faulting, Annu. Rev. Earth Planet. Sci., 26, 643-696.

Marone, C., C. B. Raleigh, and C. H. Scholz (1990), Frictional behavior and constitutive modeling of simulated fault gouge, J. Geophys. Res. 95, 7007-7025.

McCaffrey, R., L. M. Wallace, and J. Beavan (2008), Slow slip and frictional transition at low temperature at the Hikurangi subduction zone, Nat. Geosci., 1, 316-320.

Miller, M. M., T. Melbourne, D. J. Johnson, and W. Q. Sumner (2002), Periodic slow earthquakes from the Cascadia subduction zone, Science, 295(5564), 2423.

Miyazaki, S., P. Segall, J. J. McGuire, T. Kato, and Y. Hatanaka (2006), Spatial and temporal evolution of stress and slip rate during the 2000 Tokai slow earthquake, J. Geophys. Res., 111, B03409, doi:10.1029/ 2004JB003426.

Montgomery-Brown, E. K., P. Segall, and A. Miklius (2009), Kilauea slow-slip events: Identication, source inversions, and relation to seismicity, J. Geophys. Res., 114, B00A03, doi:10.1029/2008JB006074.

Moore, D. E., and D. A. Lockner (2007), Comparative deformation behavior of minerals in serpentinized ultramafic rocks: Application to the slabmantle interface in subduction zones, Int. Geol. Rev., 49, 401-415.

Moore, D. E., D. A. Lockner, M. Shengli, R. Summers, and J. D. Byerlee (1997), Strengths of serpentine gouges at elevated temperature, J. Geophys. Res., 102, 14,787-14,801.

Murray, J. R., and P. Segall (2005), Spatiotemporal evolution of a transient slip event on the San Andreas fault near Parkfield, California, J. Geophys. Res., 110, B09407, doi:10.1029/2005JB003651.

Nakatani, M. (2001), Conceptual and physical clarification of rate and state friction: Frictional sliding as a thermally activated rheology, J. Geophys. Res., 106, 13,347-13,380, doi:10.1029/2000JB900453.

Obara, K. (2002), Nonvolcanic deep tremor associated with subduction in southwest Japan, Science, 296, 1679-1681.

Obara, K., H. Hirose, F. Yamamizu, and K. Kasahara (2004), Episodic slow slip events accompanied by non-volcanic tremors in southwest Japan subduction zone, Geophys. Res. Lett., 31, L23602, doi:10.1029/ 2004GL020848.

Ohta, Y., J. T. Freymueller, S. Hreinsdóttir, and H. Suito (2006), A large slow slip event and the depth of the seismogenic zone in the south centra Alaska subduction zone, Earth Planet. Sci. Lett., 247, 108-116.

Ozawa, S., S. Miyazaki, Y. Hatanaka, T. Imakiire, M. Kaidzu, and M. Murakami (2003), Characteristic silent earthquakes in the eastern part of the Boso peninsula, Central Japan, Geophys. Res. Lett., 30(6), 1283, doi:10.1029/2002GL016665.

Ozawa, S., H. Suito, and M. Tobita (2007), Occurrence of quasi-periodic slow-slip off the east coast of the Boso peninsula, central Japan, Earth Planets Space, 59, 1241-1245.

Peacock, S. M., K. Wang, and A. M. McMahon (2002), Thermal structure and metamorphism of subducting oceanic crust: Insight into Cascadia intraslab earthquakes, in The Cascadia Subduction Zone and Related Subduction Systems: Seismic Structure, Intraslab Earthquakes and Processes, and Earthquake Harzards, edited by S. Kirby, K. Wang, and S. Dunlop, U.S. Geol. Surv. Open File Rep., 02-328, 123-126.

Perfettini, H., and J.-P. Ampuero (2008), Dynamics of a velocity strengthening fault region: Implications for slow earthquakes and postseismic slip, J. Geophys. Res., 113, B09411, doi:10.1029/2007JB005398.

Rice, J. R. (1975), On the stability of dilatant hardening for saturated rock masse, J. Geophys. Res., 80, 1531-1536.

Rice, J. R. (1993), Spatio-temporal complexity of slip on a fault, J. Geophys. Res., 98, 9885-9907.

Rice, J. R. (2006), Heating and weakening of faults during earthquake slip, J. Geophys. Res., 111, B05311, doi:10.1029/2005JB004006.

Rice, J. R., and D. A. Simons (1976), Stabilization of spreading shear faults by coupled deformation-diffusion effects in fluid-infiltrated porous materials, J. Geophys. Res., 81(29), 5322-5334.

Rogers, G., and H. Dragert (2003), Episodic tremor and slip on the Cascadia subduction zone: The chatter of silent slip, Science, 300(5627), 1942-1943.

Rubin, A. M. (2008), Episodic slow slip events and rate-and-state friction, J. Geophys. Res., 113, B11414, doi:10.1029/2008JB005642.

Rubin, A. M., and J.-P. Ampuero (2005), Earthquake nucleation on (aging) rate- and-state faults, J. Geophys. Res., 110, B11312, doi:10.1029/ 2005JB003686.
Rubin, A. M., and J.-P. Ampuero (2009), Self-similar slip pulses during rate-and-state earthquake nucleation, J. Geophys. Res., 114, B11305, doi:10.1029/2009JB006529.

Rudnicki, J. W. (1979), The stabilization of slip on a narrow weakening fault zone by coupled deformation-pore fluid diffusion, Bull. Seismol. Soc. Am., 69(4), 1011-1026.

Ruina, A. (1980), Friction laws and instabilities: A quasistatic analysis of some dry frictional behavior, Ph.D. thesis, Brown Univ., Providence, R. I.

Ruina, A. (1983), Slip instability and state variable friction laws, J. Geophys. Res., 88, 10,359-10,370.

Sagiya, T. (2004), Interplate coupling in the Kanto district, central Japan, and the Boso Peninsula silent earthquake in May 1996, Pure Appl. Geophys., 161, 2327-2342.

Schmitt, S. V., and P. Segall (2008), Shear heating-induced thermal pressurization during the nucleation of earthquakes, Eos Trans. $A G U$ 89(53), Fall Meet. Suppl., Abstract T21D-08.

Schmitt, S. V., P. Segall, and T. Matsuzawa (2007), Thermal pressurization is significant during earthquake nucleation, before seismic slip, Eos Trans. $A G U, 88(52)$, Fall Meet. Suppl., Abstract S12B-02.

Schulz, W. H., G. Wang, J. P. McKenna, and F. Wang (2008), Evidence of dilatant strengthening as a mechanism controlling landslide velocity, Eos Trans. $A G U, 89(53)$, Fall Meet. Suppl., Abstract H43I-03.

Schwartz, S. Y., and J. M. Rokosky (2007), Slow slip events and seismic tremor at circum-Pacific subduction zones, Rev. Geophys., 45, RG3004, doi:10.1029/2006RG000208.

Segall, P. (2010), Earthquake and Volcano Deformation, 424 pp., Princeton Univ. Press, Princeton, N. J.

Segall, P., and A. M. Bradley (2009), Numerical models of slow slip and dynamic rupture including dilatant stabilization and thermal pressurization, Eos Trans. $A G U, 90(52)$, Fall Meet. Suppl., Abstract T22B-08

Segall, P., and J. R. Rice (1995), Dilatancy, compaction, and slip instability of a fluid saturated fault, J. Geophys. Res., 100, 22,155-22,171.

Segall, P., and J. R. Rice (2006), Does shear heating of pore fluid contribute to earthquake nucleation?, J. Geophys. Res., 111, B09316, doi:10.1029/ 2005JB004129.

Segall, P., and A. Rubin (2007), Dilatancy stabilization of frictional sliding as a mechanism for slow slip events, Eos Trans. $A G U, 88(52)$, Fall Meet. Suppl., Abstract T13F-08.

Segall, P., E. K. Desmarais, D. Shelly, A. Miklius, and P. Cervelli (2006), Earthquakes triggered by silent slip events on Kilauea Volcano, Hawaii, Nature, 442, 71-74, doi:10.1038/nature04938

Segall, P., A. Rubin, J. R. Rice, and S. Schmitt (2008), Dilatancy stabilization vs thermal pressurization as a mechanism for controlling slow vs fast slip, Eos Trans. AGU, 89(53), Fall Meet. Suppl., Abstract U32A-06.

Shelly, D. R., G. C. Beroza, S. Ide, and S. Nakamula (2006), Lowfrequency earthquakes in Shikoku, Japan, and their relationship to episodic tremor and slip, Nature, 442, 188-191.

Shelly, D. R., G. C. Beroza, and S. Ide (2007), Non-volcanic tremor and low-frequency earthquake swarms, Nature, 446, 305-307.

Shibazaki, B., and Y. Iio (2003), On the physical mechanism of silent slip events along the deeper part of the seismogenic zone, Geophys. Res. Lett., 30(9), 1489, doi:10.1029/2003GL017047.

Shibazaki, B., and T. Shimamoto (2007), Modeling of short-interval silent slip events in deeper subduction interfaces considering the frictional properties at the unstable-stable transition regime, Geophys. J. Int. $171,191-205$

Shimamoto, T. (1986), Transition between frictional slip and ductile flow for halite shear zones at room temperature, Science, 231(4739), 711-714

Sleep, N. H. (1997), Application of a unified rate and state friction theory to the mechanics of fault zones with strain localization, J. Geophys. Res. 102, 2875-2895.

Suzuki, T., and T. Yamashita (2009), Dynamic modeling of slow earthquakes based on thermoporoelastic effects and inelastic generation of pores, J. Geophys. Res., 114, B00A04, doi:10.1029/2008JB006042.

Szeliga, W., T. Melbourne, M. Santillan, and M. Miller (2008), GPS constraints on 34 slow slip events within the Cascadia subduction zone, 1997-2005, J. Geophys. Res., 113, B04404, doi:10.1029/2007JB004948.

Taylor, M. A. J. (1998), Stressing, seismicity and rupture dynamics of subduction earthquakes, Ph.D. thesis, Eng. and Appl. Sci., Harvard Univ., Cambridge, Mass

Taylor, M. A. J., and J. R. Rice (1998), Dilatant stabilization of subduction earthquake rutpure into shallow thrust interface, Eos Trans. $A G U, 79(45)$, Fall Meet. Suppl., F631.

Wech, A. G., K. C. Creager, and T. I. Melbourne (2009), Seismic and geodetic constraints on Cascadia slow slip, J. Geophys. Res., 114, B10316, doi:10.1029/2008JB006090. 
Wibberly, A. J., and T. Shimamoto (2003), Internal structure and permeability of major strike-slip fault zones: The Median Tectonic Line in Mie prefecture, southwest Japan, J. Struct. Geol., 25, 59-78.

A. M. Bradley, Computational and Mathematical Engineering, Stanford University, Stanford, CA 94305, USA.
J. R. Rice, Department of Earth and Planetary Sciences, Harvard University, Cambridge, MA 02138, USA.

A. M. Rubin, Department of Geosciences, Princeton University, Princeton, NJ 08544, USA.

P. Segall, Geophysics Department, Stanford University, Stanford, CA 94305, USA. (segall@stanford.edu) 